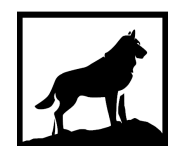

Michigan

Technological

1 8 8 5 University
Michigan Technological University

Digital Commons @ Michigan Tech

\title{
ANTI-INFLAMMATORY AND IMMUNE-MODULATING EFFECTS OF RICE CALLUS SUSPENSION CULTURE AND BLUEBERRY EXTRACT ON AN IN-VITRO INFLAMMATORY BOWEL DISEASE MODEL
}

Kyle Michael Driscoll

Michigan Technological University, kmdrisco@mtu.edu

Copyright 2017 Kyle Michael Driscoll

\section{Recommended Citation}

Driscoll, Kyle Michael, "ANTI-INFLAMMATORY AND IMMUNE-MODULATING EFFECTS OF RICE CALLUS SUSPENSION CULTURE AND BLUEBERRY EXTRACT ON AN IN-VITRO INFLAMMATORY BOWEL DISEASE MODEL", Open Access Dissertation, Michigan Technological University, 2017.

https://doi.org/10.37099/mtu.dc.etdr/497

Follow this and additional works at: https://digitalcommons.mtu.edu/etdr

Part of the Alternative and Complementary Medicine Commons, Chemicals and Drugs Commons, and the Digestive System Diseases Commons 
ANTI-INFLAMMATORY AND IMMUNE-MODULATING EFFECTS OF RICE CALLUS SUSPENSION CULTURE AND BLUEBERRY EXTRACT ON AN INVITRO INFLAMMATORY BOWEL DISEASE MODEL

\author{
By \\ Kyle M. Driscoll \\ A DISSERTATION \\ Submitted in partial fulfillment of the requirements for the degree of \\ DOCTOR OF PHILOSOPHY \\ In Biological Sciences
}

MICHIGAN TECHNOLOGICAL UNIVERSITY

2017

(C) 2017 Kyle Driscoll 
This dissertation has been approved in partial fulfillment of the requirements for the Degree of DOCTOR OF PHILOSOPHY in Biological Sciences.

Department of Biological Sciences

\author{
Dissertation Advisor: $\quad$ Rupali Datta \\ Dissertation Co-Advisor: $\quad$ Ramakrishna Wusirika
}

Committee Member: $\quad$ Aparna Deshpande

Committee Member: Tarun Dam

Department Chair: Chandrashekhar Joshi 


\section{Table of Contents}

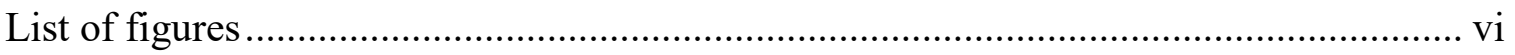

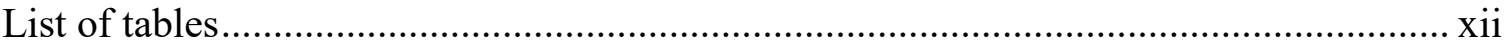

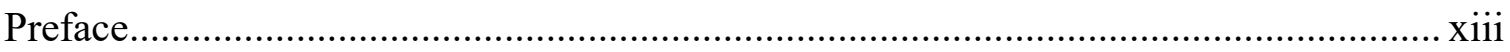

Acknowledgements .............................................................................................. xiv

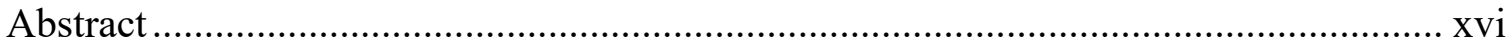

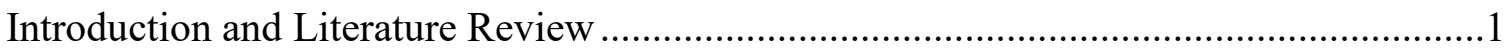

Chapter 1: Anti-Inflammatory Effects of Rice Callus Suspension Culture (RCSC) on Inflamed Colon Epithelial Cell Lines .........................................................................

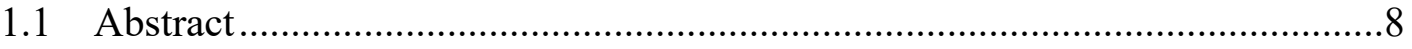

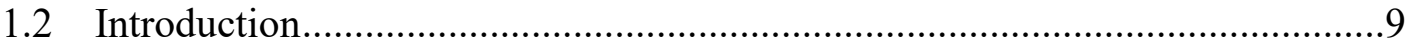

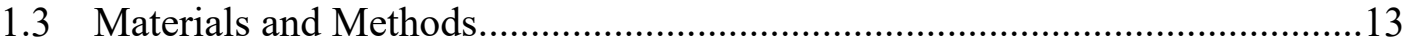

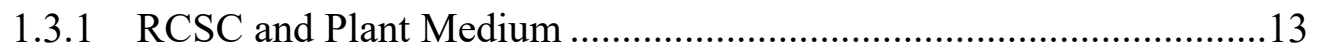

1.3.2 Human colon cell lines and culturing ...............................................13

1.3.3 Cell counting, freezing, and seeding protocol ....................................14

1.3.4 Treatment with pro-inflammatory cytokine cocktail...........................15

1.3.5 Treatment of normal colon cell lines with RCSC and PM .................16

1.3.6 Fluorescent Microscopy .................................................................16

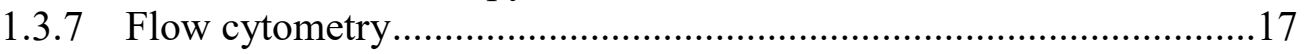

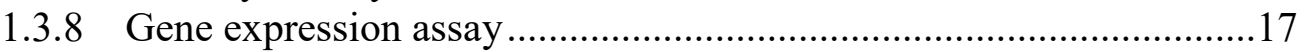

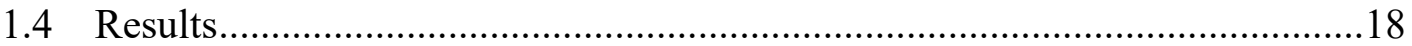

1.4.1 RCSC treatment reduced cytoplasmic ROS in InEpC, while increasing in NCM 356 and CCD $841 \mathrm{CoN}$, and enhanced nuclear ROS in all inflamed cell lines based on fluorescent microscopy analysis

1.4.2 Flow cytometry analysis identified higher levels of nuclear and cytoplasmic ROS in inflamed NCM 356 and CCD 841 CoN cell lines treated

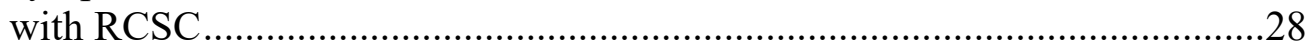

1.4.3 RCSC treatment reduced the effect of inflammation on cell death in NCM 356 and CCD $841 \mathrm{CoN}$

1.4.4 Genes differentially regulated with RCSC treatment identified by quantitative real-time PCR

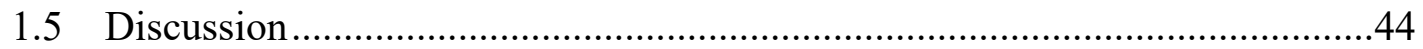

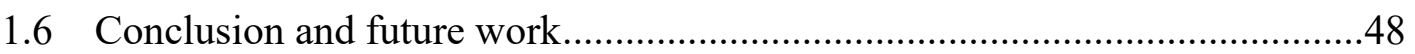

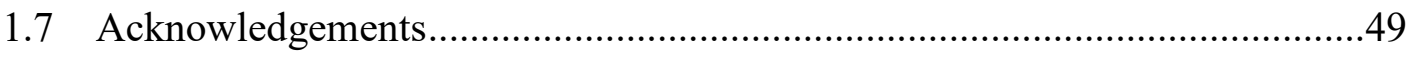


Chapter 2: Isolation of Bioactive Fractions from Rice Callus Suspension Culture (RCSC)51

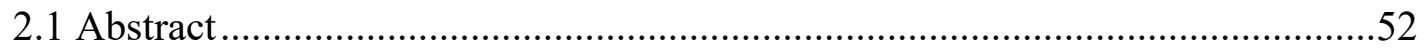

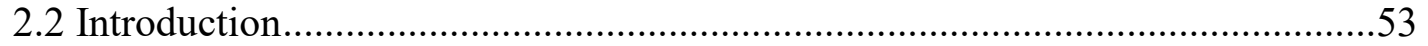

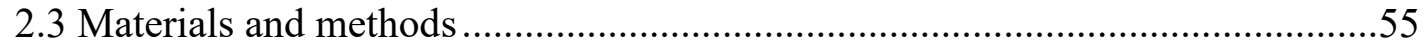

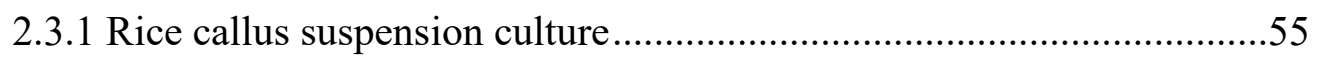

2.3.2 Preparation of RCSC for phytochemical analysis and thin layer

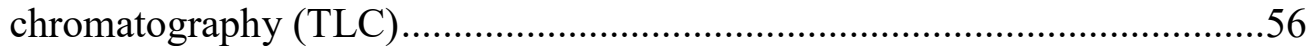

2.3.3 Column chromatography ..................................................................56

2.3.4 High Performance Liquid Chromatography (HPLC) .............................56

2.3.5 Human colon adenocarcinoma cell line SW620 culturing, passaging,

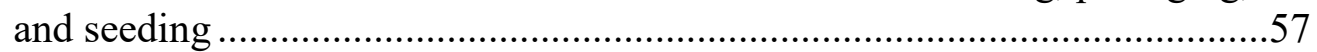

2.3.6 Normal human colon cell lines culturing, passaging, and seeding.........58

2.3.7 Treatment with pro-inflammatory cytokine cocktail.............................59

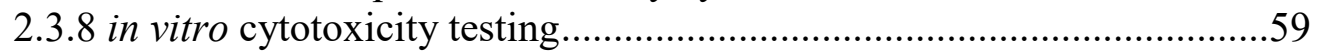

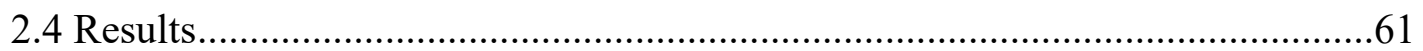

2.4.1 TLC plates identified spots in RCSC with different Rf values .............61

2.4.2 Fractionation of RCSC with column chromatography and HPLC .........64

2.4.3 RCSC and concentrates induced rapid cell death in human colon adenocarcinoma cell line SW620 …….........................................................71

2.4.4 RCSC and fractions 10-18 significantly increased cell death in human

colon adenocarcinoma cell line SW620 comparable to whole RCSC ...........74

2.4.5 RCSC and concentrates mildly increased cell death in normal human colon cell line CCD $841 \mathrm{CoN}$

2.4.5 RCSC fractions 10-18 resulted in mild increases in cell death, but showed less toxicity than whole RCSC on the normal human colon cell line

CCD $841 \mathrm{CoN}$

2.4.6 RCSC fractions 10-18 increased nuclear and cytoplasmic ROS in the normal human colon cell line CCD $841 \mathrm{CoN}$................................................ 80

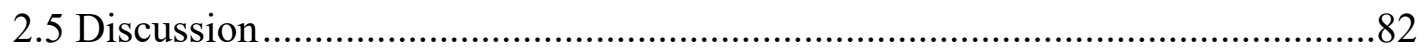

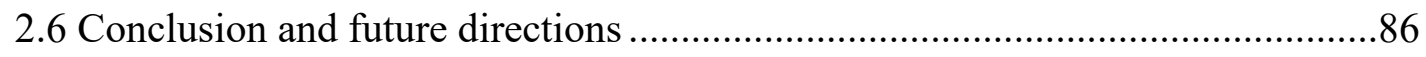

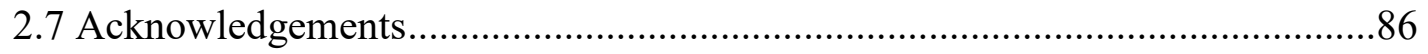

Chapter 3: Anti-inflammatory Effects of Northern Highbush Blueberry Extract on Two

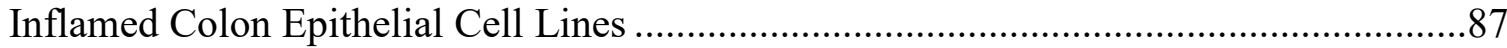

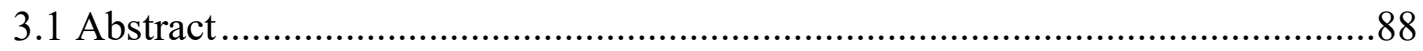

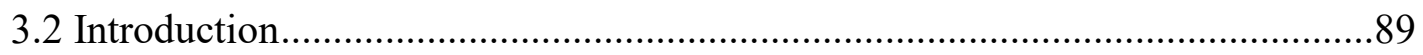

3.2.1 Inflammation and Inflammatory Bowel Disease (IBD) ........................89

3.2.2 Anti-inflammatory effects of northern highbush blueberry extract

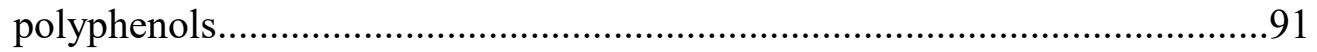

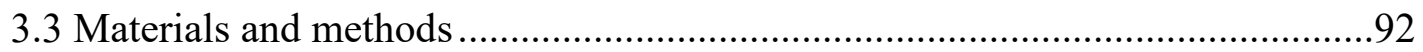




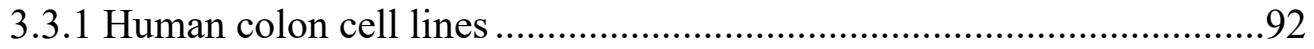

3.3.2 Cell counting, freezing, and seeding protocol ......................................92

3.3.3 Phenolic rich blueberry extract.......................................................93

3.3.4 Treatment of normal human colon cell lines with pro-inflammatory cytokine cocktail and blueberry extract.......................................................94

3.3.5 Fluorescent microscopy...................................................................94

3.3.6 Flow cytometry ................................................................................95

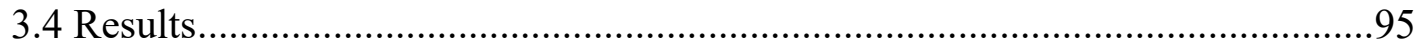

3.4.1 Blueberry extract significantly reduced nuclear and cytoplasmic ROS in inflamed human colon epithelial cells based on fluorescent microscopy ......96 3.4.2 Blueberry extract significantly reduced nuclear and cytoplasmic ROS in inflamed colon epithelial cells based on flow cytometry analysis ................103 3.4.3 Bluberry Exract Significantly reduced cell death following treatment with the pro-inflammatory cytokine cocktail based on flow cytometry analysis 111

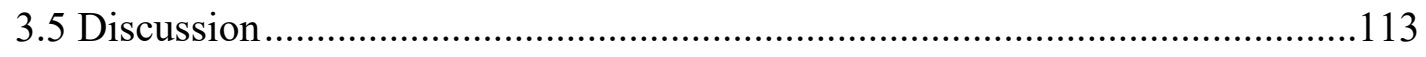

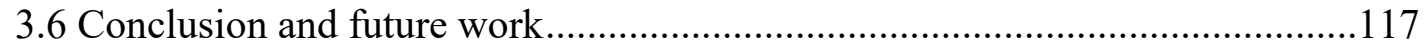

3.7 Acknowledgements.......................................................................................117

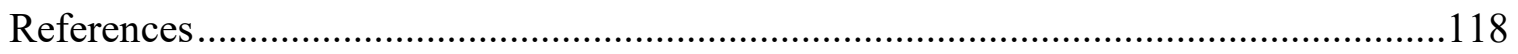

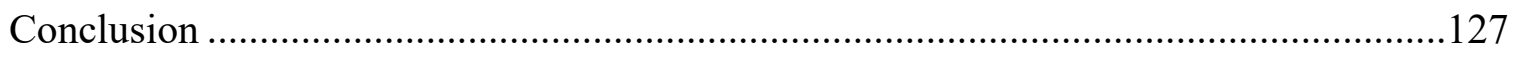

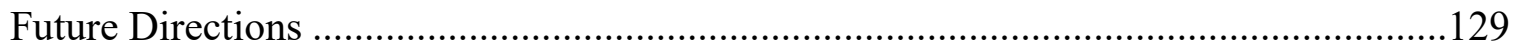




\section{List of figures}

Figure 1.1: RCSC modulates nuclear ROS in InEpC cell line

Figure 1.2: Summary data of RCSC nuclear ROS in InEpC cell line .21

Figure 1.3: RCSC decreases cytoplasmic generated ROS in InEpC cell line .22

Figure 1.4: Summary data of RCSC cytoplasmic ROS in InEpC cell line .22

Figure 1.5: RCSC increases nuclear ROS in NCM 356 cell line .23

Figure 1.6: Summary data of RCSC nuclear ROS in NCM 356 cell line .24

Figure 1.7: RCSC increases cytoplasmic ROS in NCM 356 cell line. .25

Figure 1.8: Summary data of RCSC cytoplasmic ROS in NCM 356 cell line. 25

Figure 1.9: RCSC increases nuclear ROS in CCD $841 \mathrm{CoN}$ cell line. .26

Figure 1.10: Summary data of RCSC nuclear ROS in CCD 841 CoN cell line

Figure 1.11: RCSC increases cytoplasmic ROS in CCD 841 CoN cell line. .27

Figure 1.12: Summary data of RCSC cytoplasmic ROS in CCD 841 CoN cell line........28

Figure 1.13: RCSC increases nuclear ROS in NCM 356 cell line as analyzed by flow cytometry 30 
Figure 1.14: Summary data of RCSC nuclear ROS in NCM 356 cell line for flow cytometry 30

Figure 1.15: RCSC increases cytoplasmic ROS in NCM 356 as analyzed by flow cytometry 31

Figure 1.16: Summary data of RCSC cytoplasmic ROS in NCM 356 cell line for flow cytometry

Figure 1.17: RCSC increases nuclear ROS in CCD $841 \mathrm{CoN}$ cell line as analyzed by flow cytometry

Figure 1.18: Summary data of RCSC nuclear ROS in CCD $841 \mathrm{CoN}$ cell line for flow cytometry

Figure 1.19: RCSC mildly increases cytoplasmic ROS in CCD 841 CoN cell line as analyzed by flow cytometry .35

Figure 1.20: Summary data of RCSC cytoplasmic ROS in CCD $841 \mathrm{CoN}$ cell line for flow cytometry. .36

Figure 1.21: RCSC reduced the effects of the pro-inflammatory cytokine cocktail on cell death in NCM 356 cell line as analyzed by flow cytometry.

Figure 1.22: Summary data of reduced cell death by RCSC in NCM 356 cell line. 38

Figure 1.23: RCSC reduced the effects of the pro-inflammatory cytokine cocktail on cell death in CCD $841 \mathrm{CoN}$ cell line as analyzed by flow cytometry. 39 
Figure 1.24: Summary data of reduced cell death by RCSC in CCD $841 \mathrm{CoN}$.

Figure 1.25: Heat map evaluating selected genes from immune response fast plate........41

Figure 2.1: Images of RCSC and methanol extract....................................................64

Figure 2.2: HPLC chromatogram of the 25 fractions collected via column chromatography. 71

Figure 2.3: HPLC chromatogram of fractions 10-18 .72

Figure 2.4: RCSC induces rapid cell death on the human colon adenocarcinoma cell line SW620 as analyzed by fluorescent microscopy .73

Figure 2.5: RCSC, concentrates, and fractions 10-18 rapidly induce cell death following 30-minute treatments .75

Figure 2.6: RCSC fractions 10-18 induced rapid cell death in the human colon adenocarcinoma cell line SW620 as analyzed by fluorescent microscopy..... .77

Figure 2.7: RCSC, concentrates, and fractions 10-18 had minimal toxicity towards the normal colon cell line CCD $841 \mathrm{CoN}$

Figure 2.8: RCSC fractions 10-18 increased nuclear ROS in CCD $841 \mathrm{CoN}$ similarly to whole RCSC as analyzed by flow cytometer.

Figure 2.9: RCSC fractions 10-18 increased cytoplasmic ROS in CCD 841 CoN similarly to whole RCSC as analyzed by flow cytometer. .83 
Figure 3.1: Phenolic rich blueberry extract greatly reduced nuclear ROS in NCM 356 cell line. .101

Figure 3.2: Summary data of blueberry extract nuclear ROS in NCM 356 cell line.......101

Figure 3.3: Phenolic rich blueberry extract greatly reduced cytoplasmic ROS in NCM 356 cell line. .102

Figure 3.4: Summary data of blueberry extract cytoplasmic ROS in NCM 356 cell

line.

Figure 3.5: Phenolic rich blueberry extract reduced nuclear condensation in NCM 356 cell line. .104

Figure 3.6: Summary data of blueberry extract nuclear condensation in NCM 356 cell line. .104

Figure 3.7: Phenolic rich blueberry extract greatly reduced nuclear ROS in CCD 841

CoN cell line. .105

Figure 3.8: Summary data of blueberry extract nuclear ROS in CCD $841 \mathrm{CoN}$ .105

Figure 3.9: Phenolic rich blueberry extract greatly reduced cytoplasmic ROS in CCD 841 CoN cell line. 106

Figure 3.10: Summary data of blueberry extract cytoplasmic ROS in CCD 841 CoN cell line .107 
Figure 3.11: Blueberry extract decreases nuclear ROS in NCM 356 cell line as analyzed by flow cytometry. 109

Figure 3.12: Summary data of blueberry nuclear ROS in NCM 356 as analyzed by flow cytometry 109

Figure 3.13: Blueberry extract decreases cytoplasmic ROS in NCM 356 cell line as analyzed by flow cytometry. 110

Figure 3.14: Summary data of blueberry cytoplasmic ROS in NCM 356 as analyzed by flow cytometry

Figure 3.15: Blueberry extract decreases nuclear and cytoplasmic ROS in NCM 356 cell line as analyzed by flow cytometry

Figure 3.16: Blueberry extract decreases nuclear ROS in CCD $841 \mathrm{CoN}$ cell line as analyzed by flow cytometry.

Figure 3.17: Summary data of blueberry nuclear ROS in CCD 841 CoN cell line as analyzed by flow cytometry.

Figure 3.18: Blueberry extract decreases cytoplasmic ROS in CCD $841 \mathrm{CoN}$ cell line as analyzed by flow cytometry.

Figure 3.19: Summary data of blueberry cytoplasmic ROS in CCD 841 CoN cell line as analyzed by flow cytometry .114 
Figure 3.20: Blueberry extract reduced the effects of the pro-inflammatory cytokine cocktail on cell death in CCD $841 \mathrm{CoN}$ cell line.

Figure 3.21: Summary data of reduced cell death by blueberry extract on CCD 841

$\mathrm{CoN}$ 


\section{List of tables}

Table 1.1: Differentially regulated genes in RCSC vs. inflammatory control...................41

Table 1.2: Differentially regulated genes in RCSC vs. plant medium control.................41

Table 1.3: Differentially regulated genes in RCSC vs inflammatory control in CCD 841

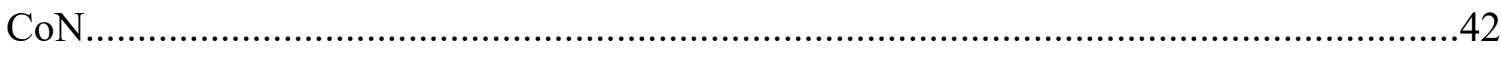

Table 1.4: Differentially regulated genes in RCSC vs. inflammation and PM controls....43

Table 1.5: Differentially regulated genes in NCM356 and CCD $841 \mathrm{CoN}$ when treated

with RCSC versus both inflammation and PM controls.............................................44 


\section{Preface}

My contributions to chapters 1 and 3 include the entirety of data collection with assistance in data analysis from Andrew D. Chapp. HPLC data was collected with assistance from Zhimin Song. All data was collected in Dr. Ramakrishna Wusirika's, Dr. Zhiying Shan, and Dr. Rupali Datta's laboratories. I wrote the initial drafts with revisions made by Dr. Ramakrishna Wusirika to create the final product. Chapter 2 was developed by Dr. Ramakrishna Wusirika and Dr. Aparna Deshpande. My contributions include the entirety of data collection with assistance in data analysis from Andrew D. Chapp. I wrote the initial drafts, with revisions made by Dr. Ramakrishna Wusirika to create the final product. RCSC was made by a previous student, Dr. Surendar Reddy Dhadi. Initial training in experimental techniques was provided by Dr. Aparna Deshpande and Dr. Ramakrishna Wusirika. Most of the work was supported by a grant from the National

Institute of Food and Agriculture, U.S. Department of Agriculture to Dr. Aparna Deshpande, Dr. Rupali Datta and Dr. Ramakrishna Wusirika. 


\section{Acknowledgements}

First and foremost I would like to thank Les Cook, and Dr. Mike and Dr. Susan Skochelak for the generous financial gift, which has helped me tremendously along the way. I would like to also the biological sciences department for the support and funding I have received.

I would like to express my sincerest gratitude to my advisors, co-advisors and committee memebers; Dr. Ramakrishna Wusirika, Dr. Aparna Deshpande, Dr. Rupali Datta, and Dr. Tarun Dam whom have helped immensely to shape who I am today, beginning with my undergraduate career to where I am now. Without their continuing support, patience, understanding and immense knowledge from near and afar, I would not have accomplished what I have, nor would this dissertation be complete.

I would like to thank the helpful staff members of the department; Jeff Lewin, Patricia Asselin and Emily Betterly for their patience and understandings in times of distress and aiding in making things easier at Michigan Tech.

A special thanks goes to Dr. Qing-Hui Chen and Dr. Zhiying Shan for the vast amount of support in all areas of my research as well as with financial help.

I would especially like to thank my fellow graduate students and friends Andrew Chapp, Andrew Kennedy, Dr. Li Chen, Dr. Komal Raja, Jeffrey Kiiskila, Zichen Qian, Zhimen Song, and any other names I may have forgotten for the continual support and 
intuitive conversations along the way. The friendships I have made here will last a lifetime.

Lastly, I owe a huge debt of gratitude to my parents Timothy and Catherine Driscoll who allowed me to realize my own potential, whom have been endlessly patient, supportive, and loving. They have helped shaped me in ways words cannot explain, and I'm wholeheartedly thankful for that. 


\section{Abstract}

Rice Callus Suspension Culture (RCSC) has been shown to exhibit potent anticancer activity in vitro. In this study we aimed to investigate the anti-inflammatory and immune-modulating properties of RCSC on three inflamed normal colon epithelial cell lines, InEpC, NCM 356, and CCD $841 \mathrm{CoN}$. As a comparative, we additionally analyzed the anti-inflammatory effects of Highbush blueberries on two inflamed colon cell cultures, NCM 356 and CCD 841 CoN. Furthermore, we sought to characterize the bioactive compounds in RCSC responsible for anti-cancer, anti-inflammatory, and immune-modulating effects. This work will be an essential starting point for understanding and identifying possible bioactive compounds in the fight against inflammatory bowel disease (IBD) and various cancers.

Fluorescent microscopy, flow cytometry, and quantitative polymerase chain reaction (qPCR) were performed, coupled with various bioassays, such as nuclear and cytoplasmic reactive oxygen species scavenging capabilities, propidium iodide cell viability assay, and gene microarrays to identify immunological gene targets. In doing so both RCSC and Highbush blueberry extracts were quantified for their anti-inflammatory and immune-modulating functions.

Finally, several organic solvents and techniques were utilized to identify possible bioactive compounds present in RCSC, Rotor evaporation, centri-vaped, column chromatography, thin layer chromatography (TLC), and high performance liquid chromatography (HPLC). Because of monetary and time restrictions we were unable to 
identify the single responsible bioactive compound, if the case, however we were able to identify bioactive fractions consisting of eight compounds with anti-cancer and antiinflammatory properties with great similarities to the whole RCSC. This will serve as a starting point for characterization of the bioactive compound(s) 


\section{Introduction and Literature Review}

\section{Plant secondary metabolites}

Plants for centuries have produced a wide variety of chemicals that do not primarily participate in growth and development of an organism ${ }^{1}$. These compounds, termed secondary metabolites, are distinctly distributed among a wide range of taxonomic groups, and contribute immensely to the overall fitness of an organism ${ }^{1,2}$. Additionally, they exhibit a broad range of biological activity, such as antibacterial, antifungal, antiviral, anti-inflammatory and anti-cancerous among others ${ }^{1-3}$.

During 1981-2010, the Food and Drug Administration reported 34\% of new small molecules approved were natural products and/or derivatives ${ }^{4}$. Furthermore, more than $60 \%$ of chemotherapy and infectious disease drugs are from natural products. Their classification falls into three distinct classes, based on the route of synthesis: terpenoids, alkaloids, and phenylpropanoids and allied phenolic compounds ${ }^{1,2,5,6}$. Some examples include the terpenoid forskolin that is used to treat glaucoma, the anticancer drug vinblastine, the alkaloid morphine synthesized by Friedrich Serturner in 1806, and campthothecin, another anticancer drug, among a plethora of others ${ }^{1}$.

Each class of secondary metabolites consists of distinct chemical structures, however extensive variations do exist. Terpenoids are classified based on a repetitive fusion of branched five-carbon isoprene units ${ }^{1}$. The term "isoprene" comes from the final product of thermal degradation of terpenoids resulting in the isoprene gas ${ }^{1}$. Terpenoids are the largest and most diverse class of natural products with 64,000 known 
compounds ${ }^{1,7}$. The diversity in terpenoids structure and function has elicited great interest in possible medicinal and commercial uses ${ }^{7}$.

Alkaloids have classically been defined as biologically active, nitrogenous containing compounds derived from plants ${ }^{1}$. After roughly 200 years of research, the definition has been changed to encompass a greater diversity of compounds. First, not all alkaloids are biologically active, second alkaloids, are found in animals and other taxa, and finally not all alkaloids contain a nitrogenous atom ${ }^{1}$. Alkaloids, like the third and final class of natural products (yet to be described) can be derived from amino acids, and/or purines, and/or a derivation of terpenoids, and/or acetate precursor with a nitrogenous heterocyclic ring. To this day, a single underlying defining characteristic of the term "alkaloids" is still unknown". In saying this, around 30,000 compounds have been described as alkaloids ${ }^{8}$. These compounds have had a great influence on modern medicine, the most well known prescription alkaloid being the antitussive and analgesic, codeine. Additionally, alkaloids in recent times have had great influence in politics. The most well known example being the opium war between China and Great Britain in the years $1839-1859^{1}$.

Phenylpropanoids and allied phenolic compounds account for roughly $40 \%$ of circulating atmospheric carbon ${ }^{1}$. These compounds are characterized as metabolites, which are aromatic, and contain one or more acidic hydroxyl groups attached to a phenyl ring $^{1}$. Phenolics are another broad category of bioactive compounds mainly derived from the phenylpropanoid and phenylpropanoid acetate pathway, which utilizes the aromatic amino acids phenylalanine and tyrosine ${ }^{1}$. Additionally, an alternative metabolic process, 
the shikimate pathway, generates copolymers utilizing the derived gallic and ellagic acids $^{1}$. Several metabolites derived from these pathways are termed "flavonoids" which are comprised of over 4500 compounds ${ }^{1}$. Furthermore, flavonoids have been cited to exhibit anti-inflammatory, antiviral, antioxidant, and antineoplastic properties ${ }^{9}$. Some examples include quercetin which inhibits the cyclooxygenase pathway, and Hesperidin and Diosmin which significantly inhibit prostaglandin synthesis, acting as potent inflammatory mediators 9 .

\section{Plant exudates}

Plant exudates are compounds secreted by the roots of plants to modulate the rhizosphere, the root system and surrounding area ${ }^{10}$. This includes ions, inorganic acids, amino acids, organic acids, sugars, phenolic compound, as well as a vast array of secondary metabolites ${ }^{10}$. These compounds aid in maintaining the symbiosis between microorganisms and plants as well as preserving the overall fitness of the plant ${ }^{10}$.

Recently much work has focused on generating bioactive compounds from in vitro plant exudates. There are several benefits to utilizing plant exudates versus organic

extractions in that percent yield can be much higher then in the parent plant $^{11}$. For example, rosmarinic acid from Coleus blumei cell culture was nine fold higher then from the whole plant. Additionally, extractions from exudates are simple compared to whole plant tissue, reducing possible production costs of bioactive compounds ${ }^{11}$.

In utilizing in vitro plant exudates from rice callus, termed as rice callus suspension culture (RCSC), our research group identified bioactive compounds with 
potent anticancer properties, which upon further investigation showed selectivity towards transformed cell lines with minimal toxicity towards normal cells in vitro ${ }^{12,13}$. Specifically, RCSC was a potent anticancer agent against colon adenocarcinoma and lung cancer cell lines. Because of this, we aimed to investigate if RCSC possessed antiinflammatory properties in an in vitro inflammatory bowel disease model.

\section{Inflammatory bowel disease (IBD)}

Inflammatory bowel disease consists of two main disorders: Crohns disease (CD) and ulcerative colitis (UC) ${ }^{14-17}$. Roughly 1 million individuals in the United States have IBD, with an annual diagnosis of 30,000 individuals ${ }^{14}$. The age of onset for IBD is a bimodal distribution with the majority of cases occurring between the ages of 15-30 years and between 50-70 years ${ }^{14,15}$. Symptoms include diarrhea, abdominal cramping, anemia, weight loss and fatigue ${ }^{18}$. There is currently no cure; therefore treatment is dependent on symptom control, minimizing complications both transient and chronic, and improving quality of life ${ }^{17-19}$. While both disorders are characterized as chronic with relapsing inflammation of the intestinal epithelium, distinct pathologies do exist ${ }^{14-16}$.

Crohns disease is characterized by patchy transmural inflammation, with "skip lesions"16. Pathologically, Crohns disease contains non-caseating granulomas with macrophage aggregates ${ }^{18}$. It most commonly affects the terminal ileum, with early symptoms appearing over Peyer's patches, however any part of the gastrointestinal tract may be affected ${ }^{18}$. Ulcerative colitis is characterized by continual diffused mucosal inflammation that extends from the rectum to the proximal colon ${ }^{16,18}$. Histologically, 
abscesses and large amounts of neutrophils are present within the lamina propria and the crypts $^{18}$. Furthermore, several genetic and chemical concoctions are present. Employment of genome wide searches within the past decade or so have been highly successful in identifying genetic loci in IBD. Nucleotide Binding Oligomerization Domain Containing 2 (NOD2) has strongly been implicated in the development of Crohn's disease. Additionally, several genes responsible for epithelial barrier function seem to be major contributing factors in the pathogenesis of IBD, for example, N-cadherin mutant, Muc2, and ABCB1, among several others ${ }^{18}$. This coupled with increased cytokine production such as TNF- $\alpha$, interleukin class molecules such as IL2 and IL23, and NF- $\mathrm{KB}$ seem to promote a chronically inflamed environment ${ }^{18}$. Moreover, this environment is amplified by the invasiveness of pathogenic bacteria. In trying to phagocytize and induce autophagy on infected cells, the "leaky gut" allows the bacterial toxins to further initiate the innate and adaptive immune responses further facilitating the chronic inflammatory cycle $^{18}$.

Current treatment options revolve around the management of symptoms, and more recently immunotherapy based treatments. IBD manifests with a multitude of symptoms, which are largely due to the massive amounts of cytokines and chemokines released by the immune system. This information enabled researchers to target and create anti-antibodies. Initially, anti-inflammatory cytokines were targeted; specifically IL-10, IL-11, and IFN $\beta$, however, the results were disappointing ${ }^{20}$. A breakthrough came with a neutralizing antibody targeting TNF, infliximab. In patients with Crohns disease the results were clinically astounding, major improvements were seen in the inflamed 
tissues $^{20}$. In patients with ulcerative colitis, similar results were obtained, which started the foundational treatment for patients with $\mathrm{IBD}^{20}$. While it seemed as if we had an understanding of the biochemical processes at work, similar anti-neutralizing antibodies, such as IFN- $\gamma$, did not shown any marked improvements, in some cases symptoms were exasperated ${ }^{20}$.

Finally, while much progress has been made in treating IBD, there is still no cure. Additionally, several other drugs are still needed to control symptoms, such as corticosteroids and aminosalicylates among several others ${ }^{21}$. Aminosalicylates are of natural origin and there is a need to continue to utilize plants to search for compounds that can aid in the treatment of inflammatory diseases, such as IBD. In vitro generation of with unlimited plant secondary metabolites through callus suspension cultures exhibiting such effects, can prove to be important both economically and treatment efficiency. Fruit extracts, such as blueberries, also serve as important sources of biochemically active secondary metabolites. These in conjunction with current treatments might provide additional relief for the millions affected with IBD. 
Chapter 1: Anti-Inflammatory Effects of Rice Callus Suspension Culture (RCSC) on Inflamed Colon Epithelial Cell Lines 


\subsection{Abstract}

Rice Callus Suspension Culture (RCSC) has been shown to exhibit potent anticancer activity in colon, renal, breast, and lung cancer cell lines. Here, we investigated the anti-inflammatory potential of RCSC on three inflamed normal colon epithelial cell lines, InEpC, NCM 356, and CCD 841 CoN. Cells were treated with a pro-inflammatory cytokine cocktail for 24 hours, followed by RCSC treatment at varying dilutions for $24-96$ hours. Real time imaging utilizing cell viability and reactive oxygen species assays were performed. Fluorescent microscopy of InEpC treated with 1:5 dilution of RCSC for 24 hours showed drastic decrease in cytoplasmic reactive oxygen species (ROS), while mild increase was observed in nuclear generated ROS. NCM 356 treated with 1:40 dilution of RCSC for 48 hours showed minor increase in cytoplasmic generated ROS, while drastic increase was observed in nuclear generated ROS. CCD $841 \mathrm{CoN}$ at a 1:40 dilution of RCSC for 48 hours revealed increase in nuclear generated ROS and decrease in cytoplasmic generated ROS. Flow cytometry analysis of propidium iodide staining was suggestive of decreased cell death in RCSC treated groups in both NCM 356 and CCD 841 CoN. Several changes in gene expression were observed in NCM 356 cell line with five genes upregulated and ten genes downregulated following 48-hour treatment with RCSC versus inflammatory control. Gene expression analysis on CCD 841 CoN cell line identified nine genes upregulated and 15 genes downregulated in RCSC treated versus inflammatory control. Our studies suggest that RCSC can serve as a novel anti-inflammatory agent for the gastrointestinal tract. 


\subsection{Introduction}

The human diet consists of a plethora of chemicals, which can have significant impact on the health and overall fecundity of a population. Plants have notoriously produced a wide variety of chemicals, which have been used for therapeutic purposes dating as far back as $1400 \mathrm{BC}$ to this day ${ }^{1,22}$. Today, they are regarded as the single most promising factor in drug discovery ${ }^{22}$. Recently, within the last decade or so, between the years 2005 to 2007, thirteen drugs were approved which were naturally derived and of those thirteen, five represented newly discovered classes of drugs: peptide exenatides and ziconotides $^{22}$. Since March 2008, we have seen a 30\% decrease in the development of natural products, due to the seemingly disadvantageous nature of extraction, complex chemistry, apparent slow effects, as well as intellectual property rights ${ }^{22,23}$. Nevertheless, the wide therapeutic potential of natural products with minimal side effects has forced researchers to take a second look at them ${ }^{24}$.

The bioactivity of natural products comes from a unique class of compounds termed "secondary metabolites" $25-27$. Secondary metabolites are small in size and produced by virtually all organisms in response to biotic and abiotic stress ${ }^{26}$. Although several health benefits that have been reported through dietary consumption or supplementation, the mechanisms by which these unique compounds act are poorly understood $^{26,28,29}$. While other bioactive compounds do exist, such as saponins, 
glycosides and others, plant secondary metabolites are divided into three major classes: terpenoids, alkaloids and phenylpropanoids and allied phenolic compounds ${ }^{1,5}$.

Terpenoids consist of a five-carbon precursor, isopentenyl diphosphate, which is capable of undergoing extensive structural modifications ${ }^{1,30}$. Terpenoids are present in large amount in plants and are structurally diverse class of secondary metabolites, which are involved in a wide range of biological functions. For example, cinnamon oil has shown antimicrobial activity against Pseudomonas aeruginosa. Additionally, camphor, menthol, and pyrethrins are all examples of terpenes which act as insecticides ${ }^{30}$. Alkaloids consist of one or more nitrogenous atoms and are derived mainly from Lamino acids such as tryptophan, tyrosine, phenylalanine, lysine, and arginine ${ }^{1}$. Alkaloids can further be divided into true alkaloids, which are found in $20 \%$ of all plant species and contain nitrogenous atoms in heterocyclic rings, and pseudoalkaloids which are not derived from amino acids, but still contain nitrogenous atoms ${ }^{31}$. These metabolites have over a 3000-year history of medicinal use, from the opioid morphine, and the antiarrhythmic aimaline, to the anticholinergic atropine ${ }^{1}$. Phenylpropanoids and allied phenolic compounds consist of metabolites, which are formed through the shikimate, malonate acetate pathway, and/or the phenylpropanoid and phenylpropanoid acetate pathway ${ }^{1}$. The shikimate pathway, in a series of seven metabolic steps, begins with the condensation of phosphoenolpyruvate and erythrose-4-phosphate to yield 3-deoxy-Darabino-heptulosonate 7-phophate (DAHP) and inorganic phosphate ${ }^{6}$. In the second reaction, a phosphate is eliminated from DAHP which yields 3-dehydroquinate (DHQ), followed by dehydration to give 3-dehydroshikimate (DHS) ${ }^{6}$. DHS is then reduced to 
form shikimate, which is then phosphorylated by shikimate kinase to form shikimate 3phosphate $^{6}$. From shikimate 3-phosphate, PEP is condensed along with shikimate 3phosphate to yield 5-enolpyruvylshikimate 3-phophate and inorganic phosphate ${ }^{6}$. In the final step, 5-enolpyruvylshikimate 3-phosphate, a phosphate is removed yielding chorismate $^{6}$. From chorismate, three aromatic amino acids are formed, phenylalanine, tyrosine, and tryptophan. These three amino acids are then used in the synthesis of several proteins, as well as extensive synthesis of plant secondary metabolites ${ }^{1,6}$. The most basic examples derived from this pathway are the hydroxycinnamic acids, which consist of the basic $\mathrm{C} 6 \mathrm{C} 3$ phenylpropane skeleton. The more complex compounds include flavonoids, isoflavonoids, and stilbenes, which are formed through the condensation of the phenylpropane backbone ${ }^{6}$.

Current focus has shifted to in vitro generation of plant secondary metabolites through plant exudates. One such example is plant callus which has generated interest because of its nature of production (in vitro) compared to whole plant tissues ${ }^{11}$. Additionally, callus generation has several benefits compared to whole plants such as large scale generation of secondary metabolites without the need for growing plants in fields as well as ease of isolation and purification of products ${ }^{11}$. Callus cultures tend to be equally effective as leaf extracts as shown in case of $A$. marmelos callus culture which was as effective in management of diabetes as leaf extract ${ }^{32}$. Callus is a mass of somatic undifferentiated totipotent cells whose suspension culture has been reported to show anticancer activity on multiple cancerous cell lines, with minimal effects on normal cells $^{12,13}$. 
Chronic inflammation is tightly linked with carcinogenesis. Individuals diagnosed with inflammatory bowel disease (IBD) or chronic ulcerative colitis, have a 10 to 40 fold increased risk in developing colorectal cancer $^{33}$. Our current understanding on this transformation from a normal cell population to a neoplastic growth begins with transient exposure to biotic and abiotic stressors, which cause an immune response and release of a plethora of chemicals ranging from cytokines and chemokines to free radicals created through oxidative phosphorylation ${ }^{34,35}$. This release results in adaptive responses, stimulation of apoptosis and angiogenesis as well as induction of pre-neoplastic mutations ${ }^{34}$. While this transient response is a necessity for normal survival and function of the individual, chronic exposure leads to often-permanent neoplastic changes such as increased cell proliferation, oncogene activation, and mutagenesis ${ }^{34}$. These changes can eventually result in the formation of adenomatous polyps, and ultimately malignant adenocarcinomas $^{34}$. Our current aim is to investigate the effects of rice callus suspension culture (RCSC) on three inflamed colon epithelial cell lines: InEpC, NCM 356 and CCD $841 \mathrm{CoN}$ and to mimic the inflammatory conditions of inflammatory bowel disease (IBD) by the pro-inflammatory cytokines: interlukin 1 beta $(\mathrm{IL}-1 \beta)$, tumor necrosis factor alpha (TNF- $\alpha$ ), interferon gamma (IFN-Y), and lipopolysaccharide (LPS). 


\subsection{Materials and Methods}

Corning ${ }^{\circledR} 96$ well flat clear bottom polystyrene TC-treated micro plates, VWR ${ }^{\circledR}$ disposable serological pipets, polystyrene $5 \mathrm{ml}, 10 \mathrm{ml}$, and $25 \mathrm{ml}$ VWR ${ }^{\circledR}$ SuperClear ${ }^{\mathrm{TM}}$ ultra-high performance centrifuge tubes were used.

\subsubsection{RCSC and Plant Medium}

Plant Medium (PM) was made by supplementing Murashige and Skoog (MS) Medium (PhytoTechnology) with casamino acids, proline, and sucrose. The resulting mixture was dissolved in deionized water and autoclaved at $125^{\circ} \mathrm{C}$ for $20 \mathrm{~min}$. The cooled mixture was further supplemented with Chu's N6 vitamins (PhytoTechnology) and 2,4 dichlorophenoxyacetic acid (2,4D).

Rice calli were produced from the seeds of Oryza sativa cultivar Nipponbare grown on solid MS medium with 2,4-dichlorophenoxyacetic acid (2,4-D) and Chu's N6 vitamins (PhytoTechnology Lab) at $28^{\circ} \mathrm{C}$ as described by Deshpande et al ${ }^{17}$. The calli were incubated in the sterile liquid plant medium for three weeks. During this incubation period, compounds were secreted into the medium. RCSC was then centrifuged at 10,000 rpm for $10 \mathrm{~min}$ at $4{ }^{\circ} \mathrm{C}$ for purification and removal of debris followed by absorbance readings between $240 \mathrm{~nm}$ and $300 \mathrm{~nm}$ using Nanodrop. The supernatant was filter sterilized and aseptically aliquoted into $50 \mathrm{~mL}$ sterile culture tubes and stored at $-20^{\circ} \mathrm{C}$.

\subsubsection{Human colon cell lines and culturing}

NCM 356 was obtained and licensed for three years from INCELL (San Antonio, TX). Cells were cultured and maintained in INCELL's M300A with 10\% fetal bovine 
serum (FBS) at $37^{\circ} \mathrm{C}$ with $5 \% \mathrm{CO}_{2}$ as per manufacturer's recommendation. $\mathrm{CCD} 841$ CoN (ATCC ${ }^{\circledR}$ CRL-1790M) was obtained from ATCC (Manassas, VA) and cultured in Eagle's minimum essential medium (EMEM, ATCC® 30-2003) with $10 \%$ FBS at $37^{\circ} \mathrm{C}$ with $5 \% \mathrm{CO}_{2}$. InEpC (Clonetics ${ }^{\mathrm{TM}}$ ) normal human intestinal epithelial cell system was purchased from Lonza and maintained with $\mathrm{SmGM}^{\mathrm{TM}}-2$ BulletKit ${ }^{\mathrm{TM}}$ which includes

supplements and growth factors, insulin, hFGF-B, hEGF, FBS and gentamicin/amphotericin-B. Penicillin-Streptomycin-Amphotericin B solution (ATCC® PCS-999-002) was purchased from ATCC.

\subsubsection{Cell counting, freezing, and seeding protocol}

Cell counting was done following the general trypan blue cell exclusion assay using a hemocytometer. Cells were initially collected from a monolayer using trypsinEDTA, centrifuged and then re-suspended in appropriate cell culture medium. From there, $25 \mu \mathrm{l}$ of cells were added to $475 \mu \mathrm{l}$ of cell culture medium to generate a diluted 1:20 ratio of cells to medium. $50 \mu \mathrm{l}$ of the $1: 20$ dilution was then added to $10 \mu \mathrm{l}$ of the trypan blue stain. Finally, $10 \mu \mathrm{l}$ was utilized for cell counting on the hemocytometer. Cells were counted utilizing the following formula: Number of cells $/ \mathrm{mL}=(\mathrm{X}) *(20) *(1.2) * 10^{4}$, where $\mathrm{X}=$ average number of cells in four squares, $20=$ first dilution factor, and $1.2=$ second dilution factor. Cells were then seeded in $6,12,24$, or 96 well plates at the required cell density and incubated for 24 hours at $37^{\circ} \mathrm{C}, 5 \% \mathrm{CO}_{2}$.

Cell freezing protocol for CCD $841 \mathrm{CoN}$ was performed by detaching the cell monolayer using 3-4ml of trypsin-EDTA for 5-15 minutes, followed by addition of 6-8ml of cell culture medium to inactivate trypsin. The cells were then transferred into $50 \mathrm{ml}$ 
conical tubes and centrifuged at $125 \mathrm{x} \mathrm{g}$ for 5-10 minutes as per manufacturer recommendation. The cell pellet was then re-suspended in complete growth medium and 5\% DMSO. A stepwise reduction in temperature was employed for cell freezing, first $20^{\circ} \mathrm{C}$ for 24 hours, then $-80^{\circ} \mathrm{C}$ for an additional 24 hours, and finally liquid nitrogen for long-term storage.

NCM 356 cell line has a heterogeneous population. The floating cells and medium was collected and transferred to $15 \mathrm{ml}$ or $50 \mathrm{ml}$ conical tubes. The cells were then washed with calcium and magnesium free phosphate buffered saline (PBS) and kept in a separate $15 \mathrm{ml}$ or $50 \mathrm{ml}$ tube. Cells were then centrifuged at $500 \mathrm{x}$ g for $5-10$ minutes and re-suspended in 50-75\% complete culture medium and the magnesium/calcium free PBS. Next, $2 \mathrm{ml}$ of trypsin-EDTA was added to the monolayer and incubated at $37^{\circ} \mathrm{C}$ for $5-10$ minutes, followed by the addition of complete growth medium to neutralize the trypsin. Cells were then pooled for counting and seeding purposes. Cells were cryopreserved in INCELL's CPZTM at a 1:1 ratio to M300A. The standard stepwise freezing process was used as stated above.

\subsubsection{Treatment with pro-inflammatory cytokine cocktail}

In order to stimulate an inflammatory response in vitro, cells were treated with a

pro-inflammatory cytokine cocktail consisting of TNF- $\alpha$ (100ng/ml, Sigma), IL-1 $\beta$ (100ng/ml, Sigma), LPS (10 $\mu \mathrm{g} / \mathrm{ml}$, Sigma), and IFN- $\gamma(5 \mu \mathrm{g} / \mathrm{ml}$, Sigma) suspended in PBS, for 0-24 hours prior to any RCSC or PM treatment. 


\subsubsection{Treatment of normal colon cell lines with RCSC and PM}

When the cells reached a confluence of $70-90 \%$, they were trypsinized, and seeded in $6,12,24$, or 96 well plates at 25,000 cells per/ml for fluorescent microscopy, and 50,000-100,000 cells $/ \mathrm{ml}$ for flow cytometry. Following 24 hour treatment with the pro-inflammatory cytokine cocktail, the cells were treated with varying dilutions of RCSC and PM, 1:5, 1:10, 1:20, 1:40, 1:80, to determine the lowest effective dose. Four time points were used: $24,48,72$, and 96 hours.

\subsubsection{Fluorescent Microscopy}

NCM 356, CCD 841, and InEpC were cultured as per manufacturers' recommendations. Cells were seeded in triplicates in appropriate medium supplemented with 3\% heat-inactivated FBS at 25,000 cells per milliliter. Cells were visualized using Zeiss inverted fluorescent microscope following treatment for 24 hours with proinflammatory cytokine cocktail and 24-96 hours with RCSC or plant medium, stains for cell viability and oxidative stress. Propidium iodide (ThermoFisher Scientific) was used to assay cell viability while CellROX ${ }^{\circledR}$ Green Reagent and CellROX ${ }^{\circledR}$ Orange Reagent were used to detect nuclear and cytoplasmic oxidative stress, respectively. Because of the limited number of cells with $\mathrm{InEpC}$, only nuclear and cytoplasmic reactive oxygen species assays were performed. Cell fluorescence was quantified utilizing Image $\mathbf{J}$ software. Calculated total cell fluorescence $(\mathrm{CTCF})=$ Integrated Density $-($ Area of selected cell X Mean fluorescence of background readings). Statistical significance was determined utilizing t-test, and 2-way ANOVA with Tukey's post hoc test for pairwise 
comparison with values represented as mean \pm SEM (standard error of the mean) and results were considered significant at $\mathrm{p}<0.05$.

\subsubsection{Flow cytometry}

NCM 356 and CCD 841 CoN were seeded in 6 well Nunc ${ }^{\mathrm{TM}}$ cell culture treated dishes (Thermo Scientific), at a density $\geq 1 \times 10^{6}$ cells per milliliter, for 24 hours at $37^{\circ} \mathrm{C}$ with $5 \% \mathrm{CO}_{2}$. Cells were then treated with an inflammatory cocktail consisting of TNF- $\alpha$, IL-1 $\beta$, LPS, and IFN- $\gamma$ for an additional 24 hours prior to RCSC treatment. Following 24hour inflammatory cocktail treatment, cells were treated with RCSC or PM for 48 hours at 1:40 dilution $(0.5 \mathrm{mg} / \mathrm{ml})$. Following the above treatment, cells were assayed with propidium iodide, or ROS to determine the effects of inflammation, PM, and RCSC on cell death. Data was analyzed utilizing percentages above or below control levels. T-test and 2-way ANOVA with Tukey's post hoc test for pairwise comparison was utilized with values represented as mean $\pm \mathrm{SEM}$ and the results were considered to be significant at $\mathrm{p}<0.05$.

\subsubsection{Gene expression assay}

NCM 356 and CCD 841 CoN were grown until they were $70-90 \%$ confluent. The cells were trypsinized, collected and seeded in triplicates in six well plates at 50,000 cells $/ \mathrm{ml}$ with $3 \%$ heat-inactivated FBS in the appropriate medium. Cells were allowed 24-hour adjustment period prior to any treatment. Cells were then treated for 24 hours with pro-inflammatory cytokine cocktail prior to 24-96 hours of RCSC or PM treatments. RNA extraction was performed using Qiagen RNeasy® Mini Kit. Total RNA was 
quantified using NanoDrop spectrophotometer. Equal amount of RNA was used for cDNA synthesis using Superscript ${ }^{\circledR}$ VILO cDNA synthesis kit. TaqMan ${ }^{\circledR}$ Universal Master Mix II with UNG were used for all gene expression assays.

Four, 96-well TaqMan ${ }^{\circledR}$ Arrays for human immune response were used for each cell line. Three housekeeping genes were tested; glyceraldehyde 3-phosphate dehydrogenase (GAPDH), hypoxanthine phosphoribosyltransferase 1 (HPRT1), and betaglucuronidase (GUSB). The other 92 genes are implicated in immune function, more specifically, cell surface receptors, cell cycle and protein kinases, oxidoreductases, signal transduction, stress response, transcription factors, chemokines and cytokines along with their receptors.

\subsection{Results}

In order to quantitatively and qualitatively determine the effects of RCSC on three epithelial cell lines, we employed fluorescent microscopy and flow cytometry to determine free radical scavenging capabilities, as well propidium iodide for cell viability determination. 
1.4.1 RCSC treatment reduced cytoplasmic ROS in InEpC, while increasing in NCM 356 and CCD 841 CoN, and enhanced nuclear ROS in all inflamed cell lines based on fluorescent microscopy analysis

InEpC treatment with CellROX ${ }^{\circledR}$ green nuclear ROS reagent revealed minor increase in RCSC treated versus inflammation control (Figure 1.1 and 1.2, $\mathrm{p}<0.05$ ). InEpC cells treated with CellROX ${ }^{\circledR}$ orange probe revealed significant decrease in cytoplasmic ROS following treatment with RCSC at 1:40 dilution for 48 hours (Figure 1.3 and $1.4, \mathrm{p}<0.05)$. In most cases, treatment with the pro-inflammatory cytokine cocktail 24 hours prior to treatment resulted in higher nuclear and cytoplasmic ROS compared to control.

A.

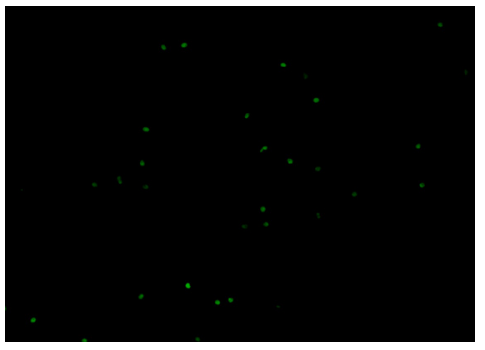

C.

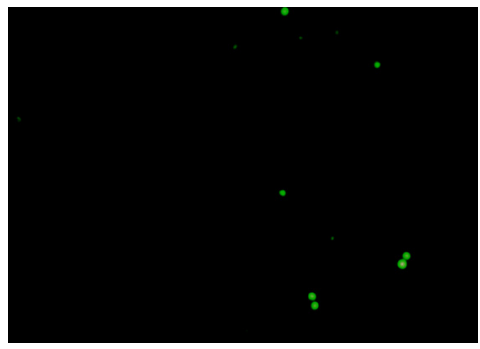

B.

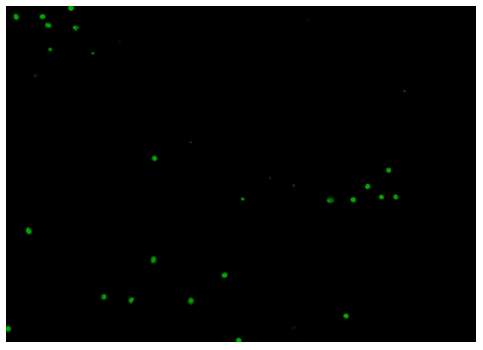

D.

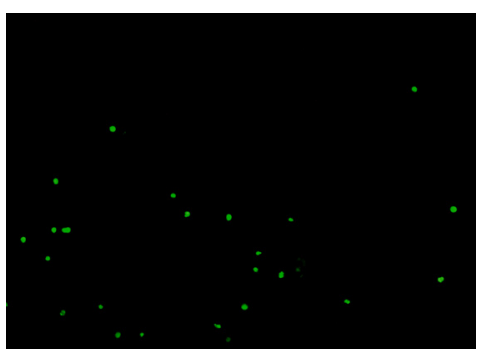

Figure 1.1: RCSC modulates nuclear ROS in InEpC cell line. InEpC cells with CellROX ${ }^{\circledR}$ green nuclear ROS staining were treated for 24 hours with the pro- 
inflammatory cytokine cocktail, followed by a 1:40 dilution of plant medium or RCSC. (A) Control (B) Inflammatory control (C) 1:40 Plant medium control and (D) 1:40 RCSC. Images were taken at 20x magnification using a Zeiss inverted fluorescent microscope.

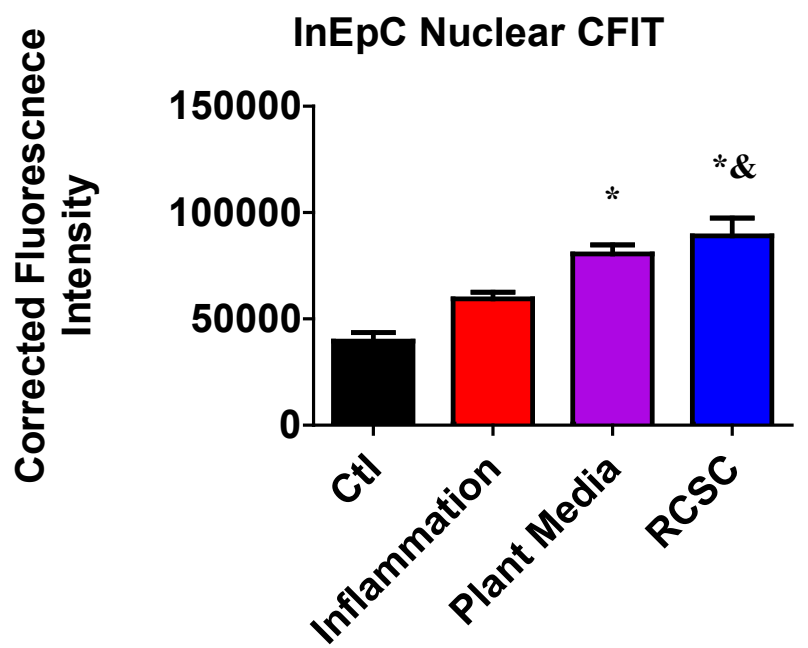

Figure 1.2: Summary data of RCSC nuclear ROS in InEpC cell line. Quantification of CellROX ${ }^{\circledR}$ green nuclear ROS staining on InEpC cell line using Image $J$ software. *= indicates significance vs. non treated control, $\&=$ indicates significance vs. inflammatory control. Results analyzed utilizing one way ANOVA with Tukeys test $(\mathrm{n}=5, \mathrm{p}<0.05)$.

A.

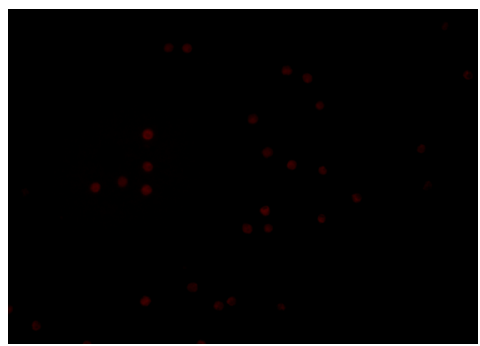

B.

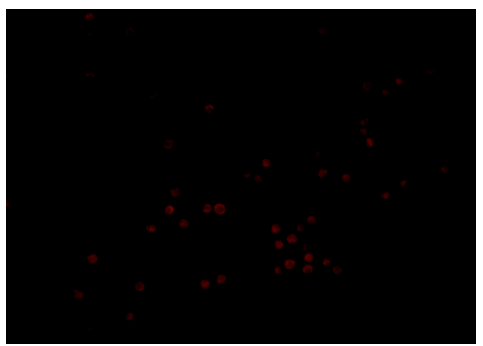


C.

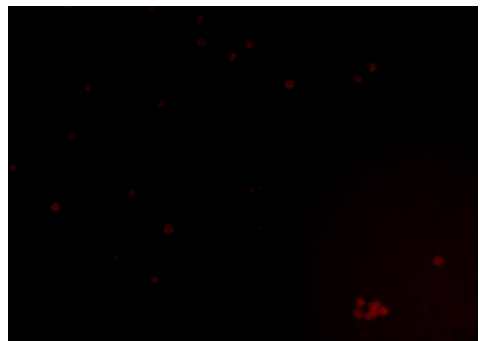

D.

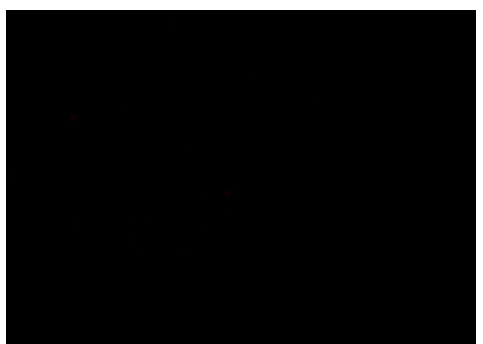

Figure 1.3: RCSC decreases cytoplasmic generated ROS in InEpC cell line. InEpC cells with CellROX ${ }^{\circledR}$ orange cytoplasmic ROS staining were treated for 24 hours with the pro-inflammatory cytokine cocktail, followed by a 1:40 dilution of plant medium or RCSC. (A) Control (B) Inflammatory control (C) 1:40 Plant medium control and (D) 1:40 RCSC. Images were taken at 20x magnification using a Zeiss inverted fluorescent microscope.

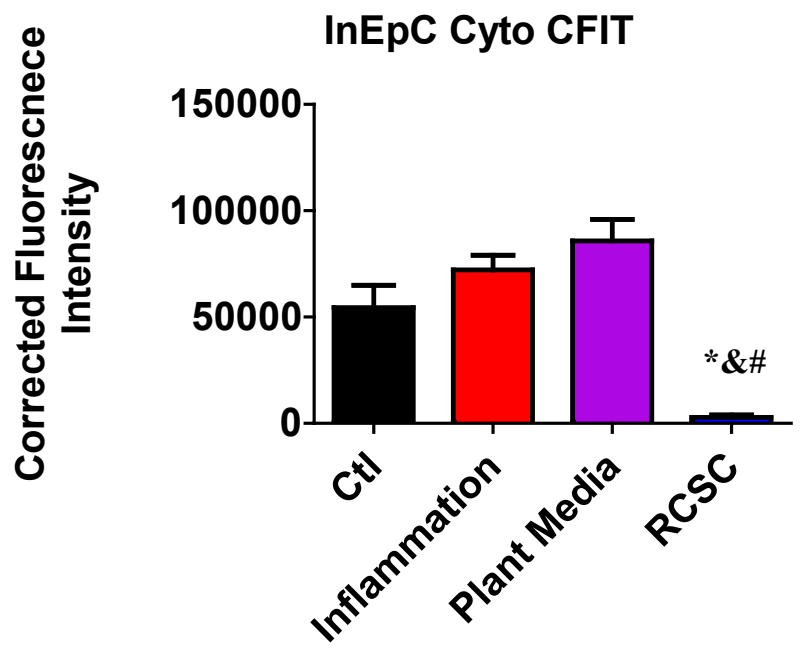

Figure 1.4: Summary data of RCSC cytoplasmic ROS in InEpC cell line. Quantification of CellROX ${ }^{\circledR}$ green nuclear ROS staining on InEpC cell line using Image J software. $*=$ indicates significance vs. non treated control, $\&=$ indicates significance vs. 
inflammatory control, \#= indicates significance vs. plant medium control. Results analyzed utilizing one way ANOVA with Tukeys test $(\mathrm{n}=5, \mathrm{p}<0.05)$.

NCM 356 (Figure 1.5 and 1.6, $\mathrm{p}<0.05$ ) and CCD $841 \mathrm{CoN}$ (Figure 1.9 and 1.10, $\mathrm{p}<0.05$ ) treatment with CellROX ${ }^{\circledR}$ green nuclear ROS probe showed significant increase $(\mathrm{p}<0.05)$ in fluorescent intensity following treatment with RCSC at 1:40 dilution for 48 hours, while treatment with CellROX ${ }^{\circledR}$ orange cytoplasmic probe again revealed significant increase in NCM 356 (Figure 1.7 and $1.8, \mathrm{p}<0.05$ ), and marginal increase in CCD $841 \mathrm{CoN}$ (Figure 1.11 and 1.12) fluorescent intensity.

A.

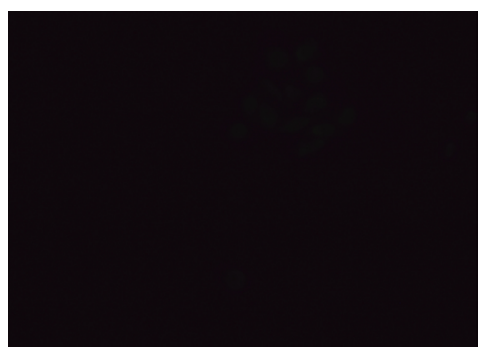

C.

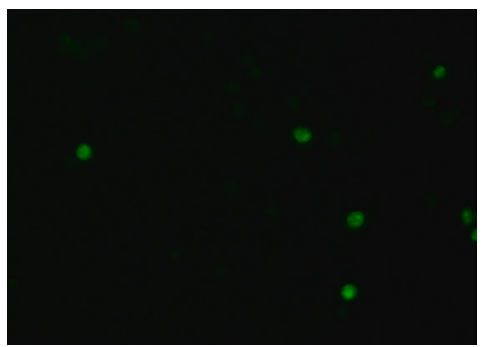

B.

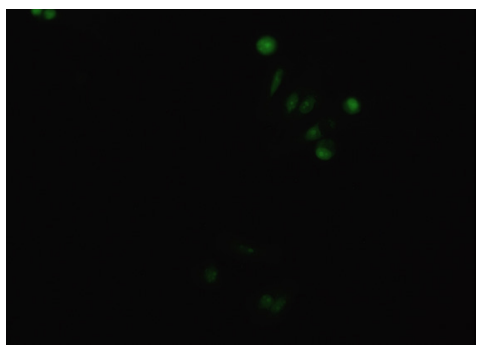

D.

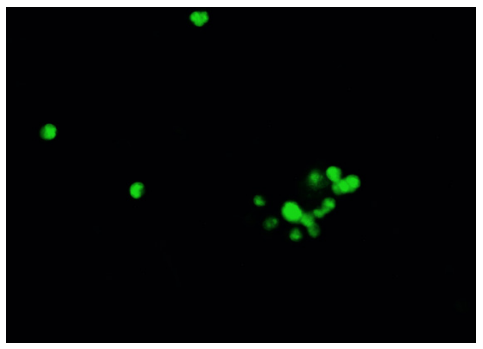

Figure 1.5: RCSC increases nuclear ROS in NCM 356 cell line. NCM 356 cells with CellROX ${ }^{\circledR}$ green nuclear ROS staining were treated for 24 hours with the proinflammatory cytokine cocktail, followed by a 1:40 dilution of plant medium or RCSC.
(A) Control
(B) Inflammatory control
(C) 1:40 Plant medium control and (D) 1:40 
RCSC. Images were taken at 20x magnification using a Zeiss inverted fluorescent microscope.

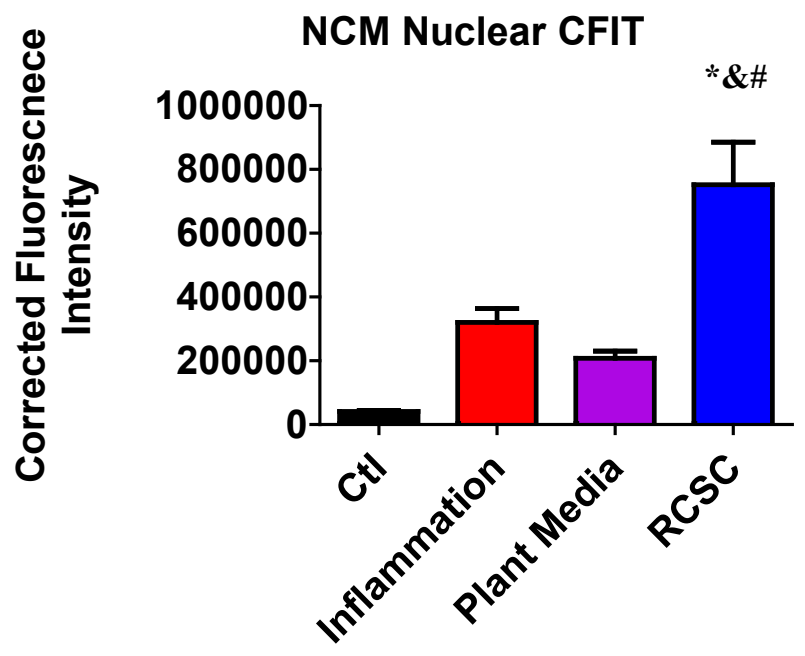

Figure 1.6: Summary data of RCSC nuclear ROS in NCM 356 cell line. Quantification of CellROX ${ }^{\circledR}$ green nuclear ROS staining on NCM 356 cell line using Image $\mathrm{J}$ software. $*=$ indicates significance vs. non treated control, $\&=$ indicates significance vs. inflammatory control, \#= indicates significance vs plant medium control. Results analyzed utilizing one way ANOVA with Tukeys test $(\mathrm{p}<0.05)$.

A.

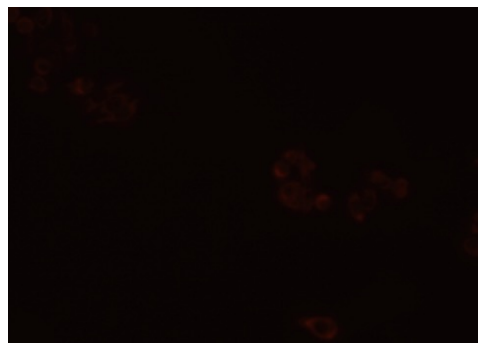

B.

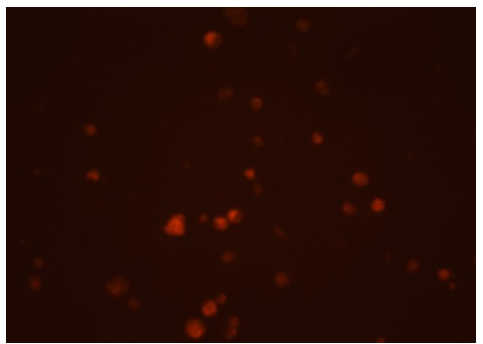


C.

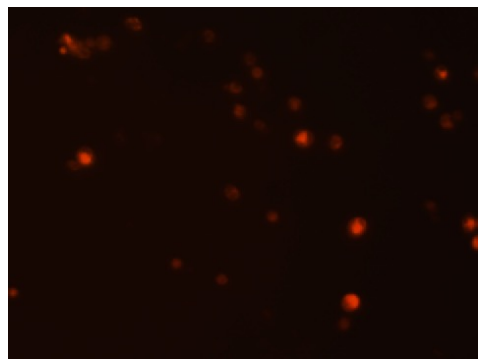

D.

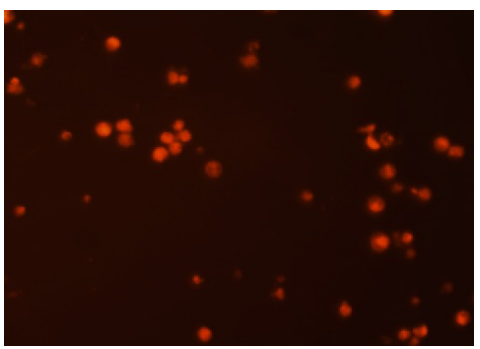

Figure 1.7: RCSC increases cytoplasmic ROS in NCM 356 cell line. NCM 356 cells with CellROX ${ }^{\circledR}$ orange cytoplasmic ROS staining were treated for 24 hours with the proinflammatory cytokine cocktail, followed by a 1:40 dilution of plant medium or RCSC. (A) Control (B) Inflammatory control (C) 1:40 Plant medium control and (D) 1:40 RCSC. Images were taken at 20x magnification using a Zeiss inverted fluorescent microscope.

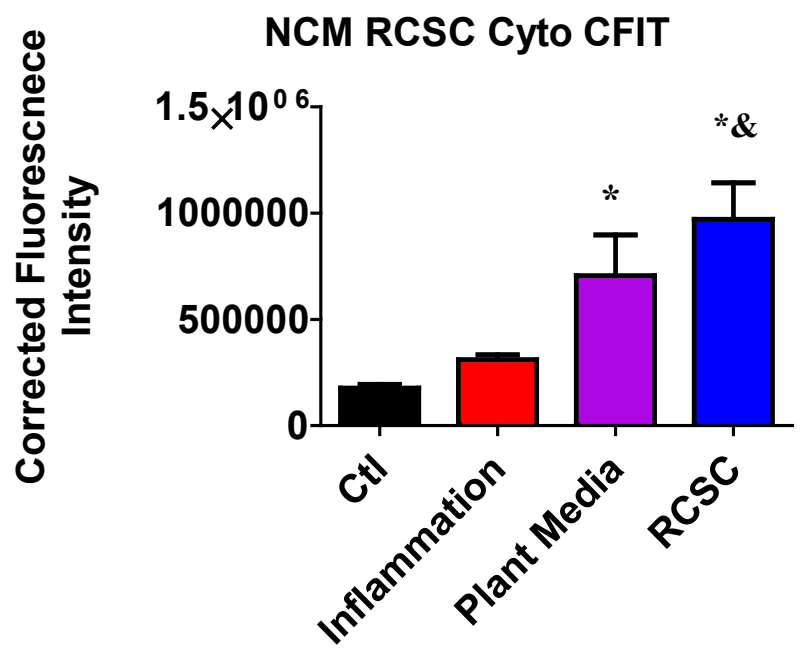

Figure 1.8: Summary data of RCSC cytoplasmic ROS in NCM 356 cell line. Quantification of CellROX ${ }^{\circledR}$ orange cytoplasmic ROS staining on NCM 356 cell line using Image $\mathrm{J}$ software. $*=$ indicates significance vs. non treated control, $\&=$ indicates 
significance vs. inflammatory control, \#= indicates significance vs plant medium control. Results analyzed utilizing one way ANOVA with Tukeys test $(n=5, p<0.05)$.

A.

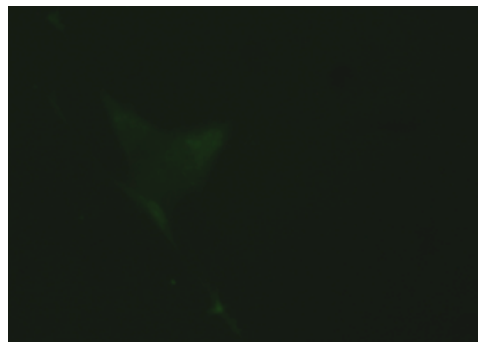

C.

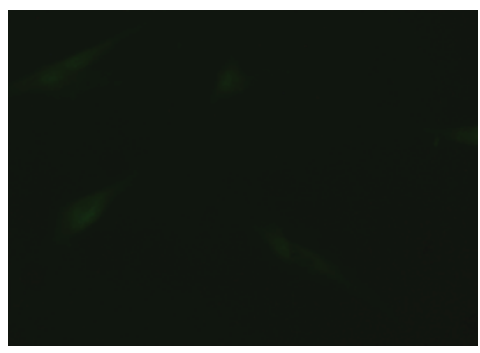

B.

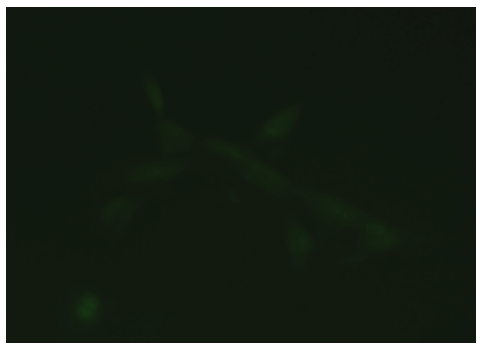

D.

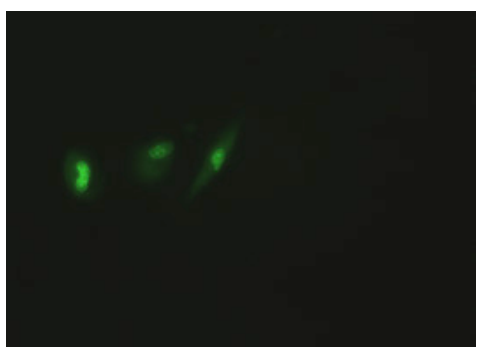

Figure 1.9: RCSC increases nuclear ROS in CCD 841 CoN cell line. CCD 841 CoN cells with CellROX ${ }^{\circledR}$ green nuclear ROS staining were treated for 24 hours with the proinflammatory cytokine cocktail, followed by a 1:40 dilution of plant medium or RCSC. (A) Control (B) Inflammatory control (C) 1:40 Plant medium control and (D) 1:40 RCSC. Images were taken at 20x magnification using a Zeiss inverted fluorescent microscope. 


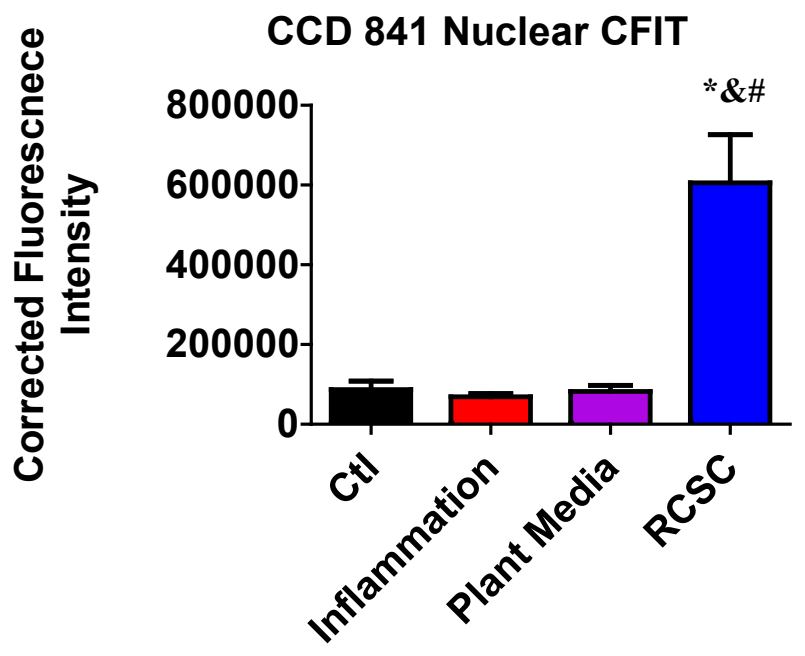

Figure 1.10: Summary data of RCSC nuclear ROS in CCD 841 CoN cell line. Quantification of CellROX ${ }^{\circledR}$ green nuclear ROS staining on NCM 356 cell line using Image $\mathrm{J}$ software. $*=$ indicates significance vs. non treated control, $\&=$ indicates significance vs. inflammatory control, \#= indicates significance vs plant medium control. Results analyzed utilizing one way ANOVA with Tukeys test $(n=5, p<0.05)$.

A.

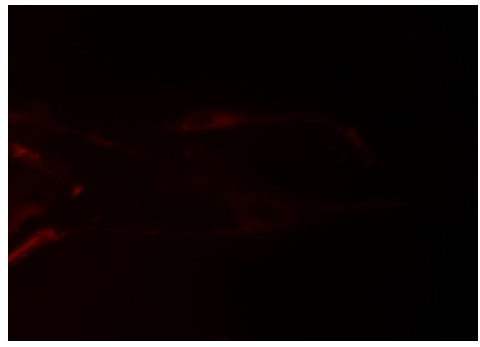

C.

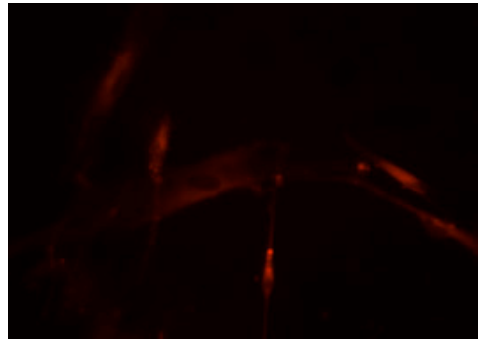

B.

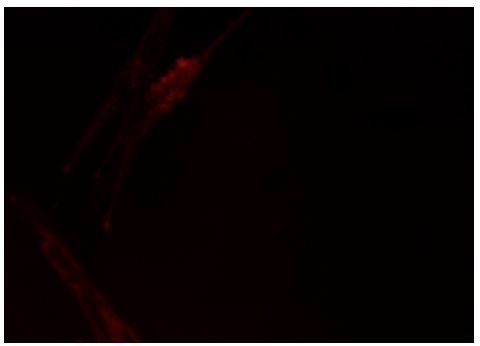

D.

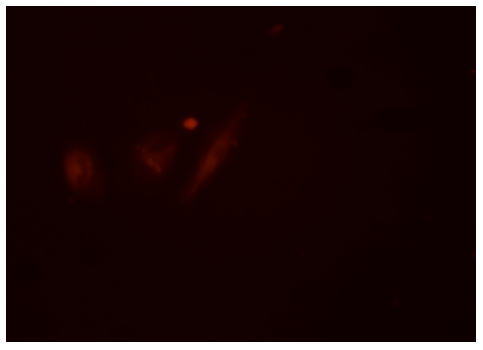


Figure 1.11: RCSC increases cytoplasmic ROS in CCD 841 CoN cell line. CCD 841 CoN cells with CellROX ${ }^{\circledR}$ orange cytoplasmic ROS staining were treated for 24 hours with the pro-inflammatory cytokine cocktail, followed by a 1:40 dilution of plant medium or RCSC. (A) Control (B) Inflammatory control (C) 1:40 Plant medium control and (D) 1:40 RCSC. Images were taken at 20x magnification using a Zeiss inverted fluorescent microscope.

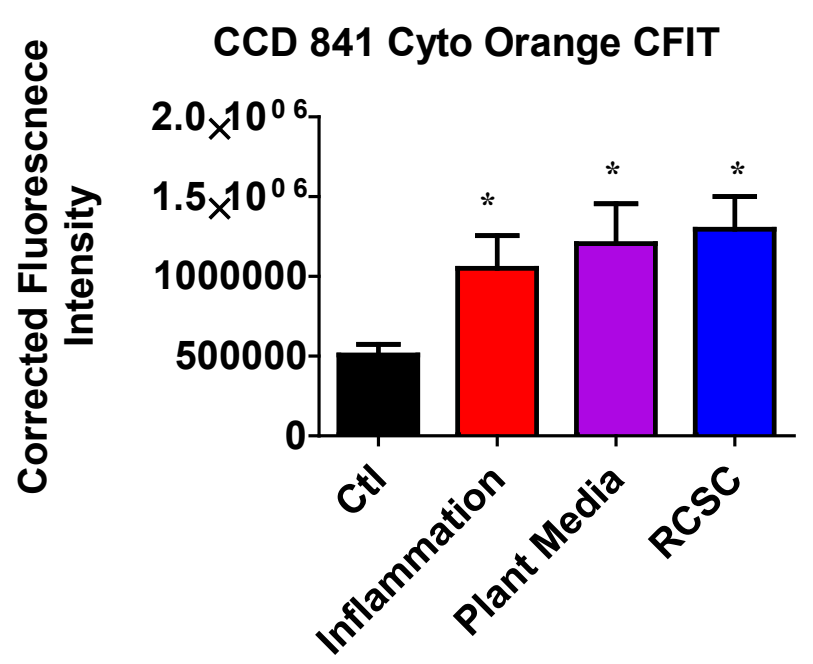

Figure 1.12: Summary data of RCSC cytoplasmic ROS in CCD 841 CoN cell line. Quantification of CellROX ${ }^{\circledR}$ orange cytoplasmic ROS staining on NCM 356 cell line using Image $\mathrm{J}$ software. $*=$ indicates significance vs. non treated control, $\&=$ indicates significance vs. inflammatory control, \#= indicates significance vs plant medium control. Results analyzed utilizing one way ANOVA with Tukeys test $(n=5, p<0.05)$. 


\subsubsection{Flow cytometry analysis identified higher levels of nuclear and cytoplasmic ROS in inflamed NCM 356 and CCD 841 CoN cell lines treated with RCSC}

NCM 356 overall morphology, as analyzed by flow cytometer, indicated increase in cell viability and forward scatter. Both forward scatter (FSC-A) and side scatter (SSCA) are dependent on the direction of light scattered by the cell. Forward scatter is able to distinguish between cell size and shape, whereas side scatter is able to detect internal complexity and granularity. NCM 356 treated with CellROX ${ }^{\circledR}$ green reagent following 48 hour RCSC treatment revealed significant increase in nuclear generated reactive oxygen species compared to control, inflammation, and plant medium with $4.2 \%, 8.2 \%$, $30.3 \%$ of cells above baseline, versus $61.4 \%$ in RCSC treatment group under FL1 (Figure 1.13 and $1.14, \mathrm{p}<0.05)$. Treatment with CellROX ${ }^{\circledR}$ orange cytoplasmic ROS probe, following the same conditions as above, revealed higher number of cells fluorescing above baseline, in RCSC treated groups compared to controls. Control, inflammation, plant medium and RCSC with $4.6 \%, 11.5 \%, 14 \%$ and $40.4 \%$ cells, respectively, above the base line were observed (Figure 1.15 and 1.16, $\mathrm{p}<0.05$ ).

A.

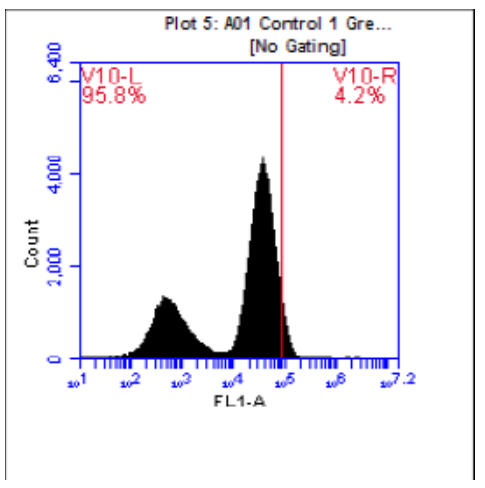

B.

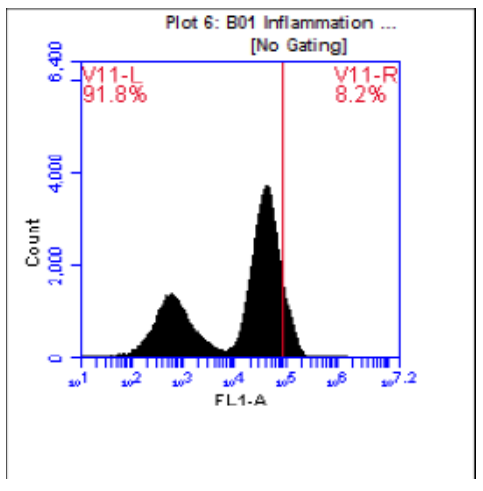


C.

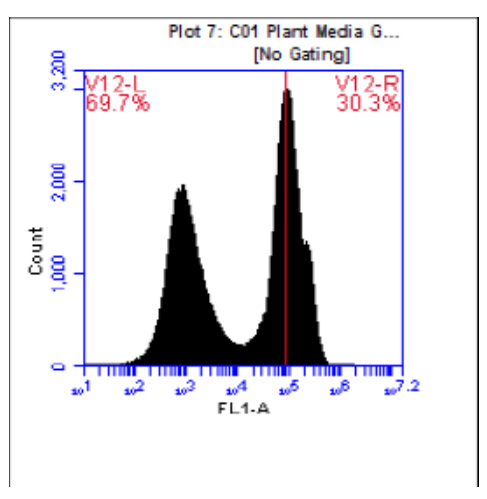

D.

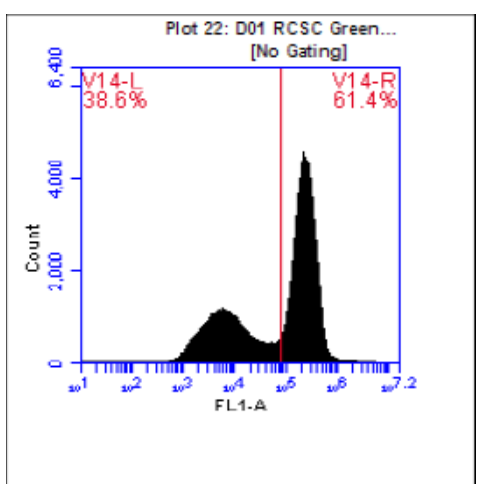

Figure 1.13: RCSC increases nuclear ROS in NCM 356 cell line as analyzed by flow cytometry. NCM 356 cells were analyzed for ROS with CellROX ${ }^{\circledR}$ green nuclear stain following 24 hour treatment with the pro inflammatory cytokine cocktail and an additional 48 hours with a 1:40 diulution of RCSC or plant medium. (A) Control (B) Inflammatory control (C) 1:40 Plant medium control and (D) 1:40 RCSC. Cells were analyzed using an BD Accuri ${ }^{\mathrm{TM}}$ C6 flow cytometer.

\section{RCSC NCM 356 Nuc ROS}

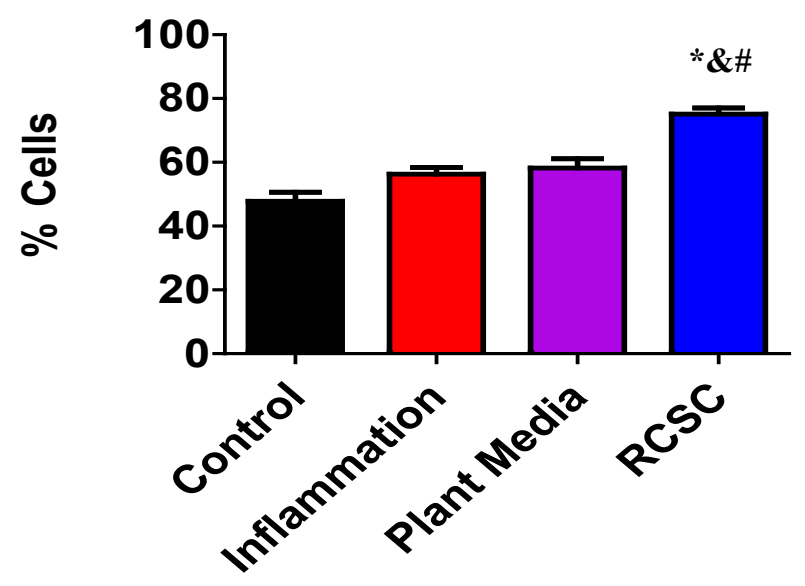

Figure 1.14: Summary data of RCSC nuclear ROS in NCM 356 cell line for flow cytometry. Quantification of CellROX ${ }^{\circledR}$ green nuclear ROS staining on NCM 356 cell 
line. $*=$ indicates significance vs. non treated control, $\&=$ indicates significance vs. inflammatory control, \#= indicates significance vs plant medium control. Results analyzed utilizing one way ANOVA with Tukeys test $(\mathrm{p}<0.05)$.

A.

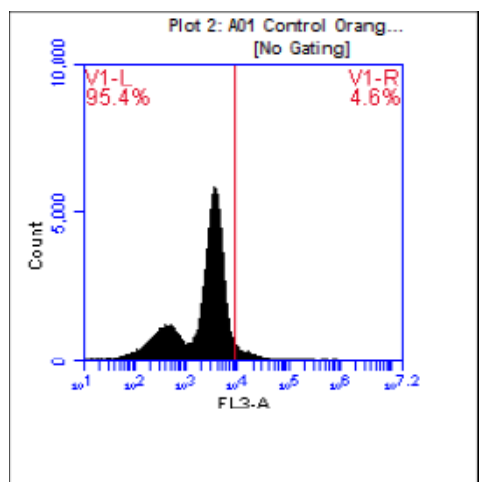

C.

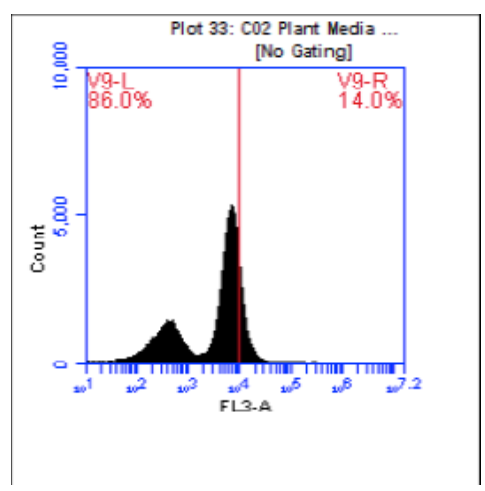

B.

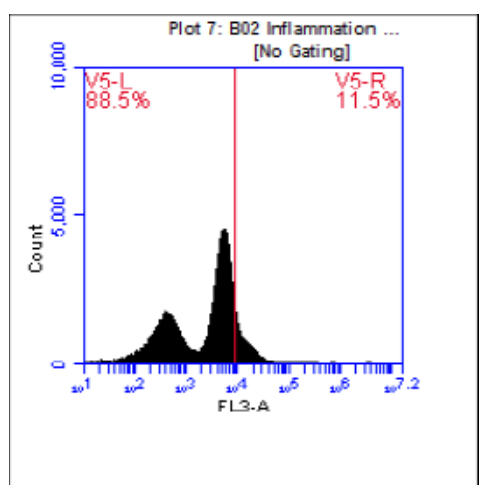

D.

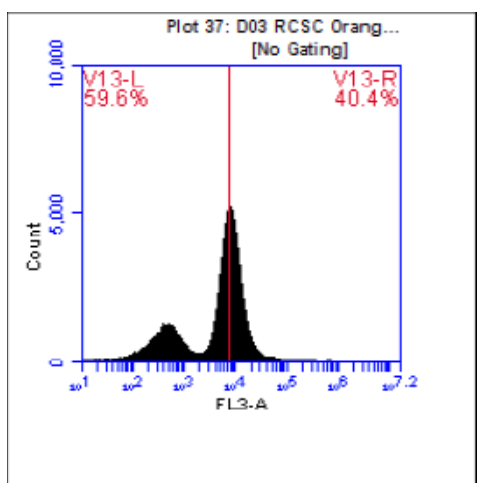

Figure 1.15: RCSC increases cytoplasmic ROS in NCM 356 cell line as analyzed by flow cytometry. NCM 356 cells were analyzed for ROS with CellROX® orange cytoplasmic stain following 24 hour treatment with the pro inflammatory cytokine cocktail and an additional 48 hours with a 1:40 diulution of RCSC or plant medium. (A) Control (B) Inflammatory control (C) 1:40 Plant medium control and (D) 1:40 RCSC. Cells were analyzed using an BD Accuri ${ }^{\mathrm{TM}}$ C6 flow cytometer. 


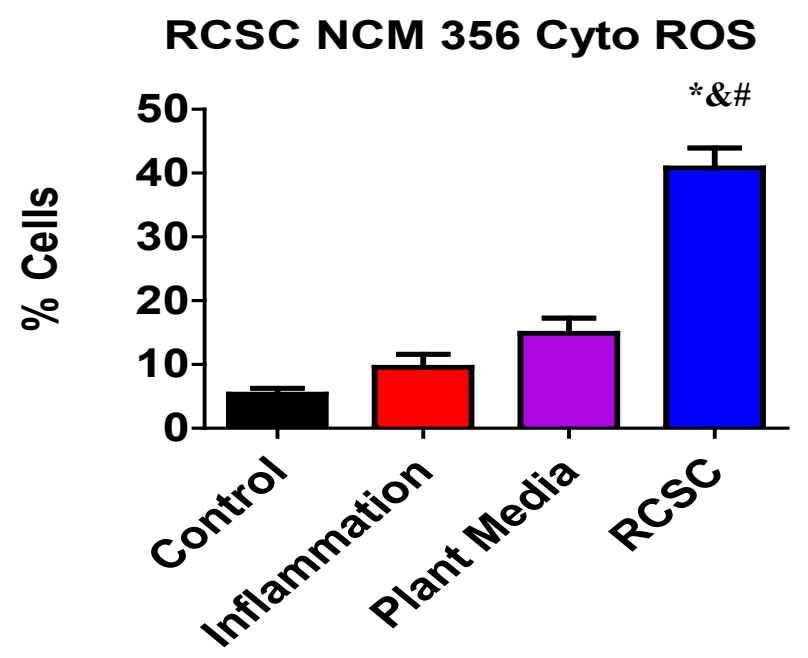

Figure 1.16: Summary data for RCSC cytoplasmic ROS in NCM 356 cell line for flow cytometry. Quantification of CellROX ${ }^{\circledR}$ orange cytoplasmic ROS staining on NCM 356 cell line. $*=$ indicates significance vs. non treated control, \&= indicates significance vs. inflammatory control, \#= indicates significance vs plant medium control. Results analyzed utilizing one way ANOVA with Tukeys test $(\mathrm{p}<0.05)$.

CCD $841 \mathrm{CoN}$ treated with CellROX $\AA$ green ROS assay displayed increase under FL1, with control, inflammation, plant medium, and RCSC treatments at $6.8 \%, 8.8 \%$, $8.7 \%$ and $32.5 \%$ fluorescent intensity, respectively above the base line (Figure 1.17 and $1.18, \mathrm{p}<0.05)$. Slight increase following treatment with $\mathrm{CellROX} \AA$ orange cytoplasmic ROS probe was observed by flow cytometer analysis with control at 3.9, inflammation and plant medium at $4.6 \%$ and $6.5 \%$, respectively, and RCSC at $9.6 \%$ of total population under FL3 (Figure 1.19 and 1.20, $\mathrm{p}<0.05$ ). 
A.

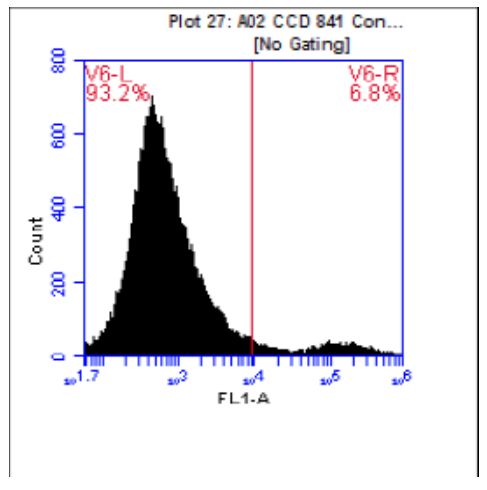

C.

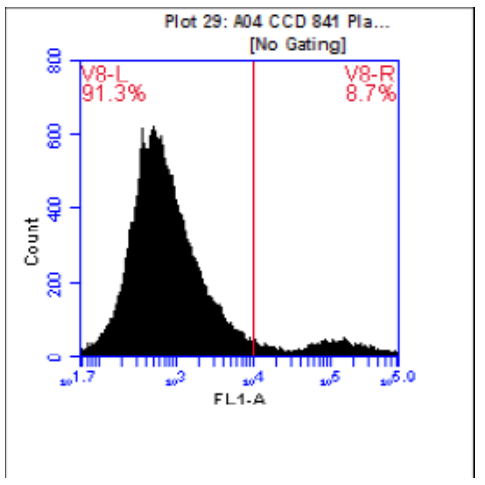

B.

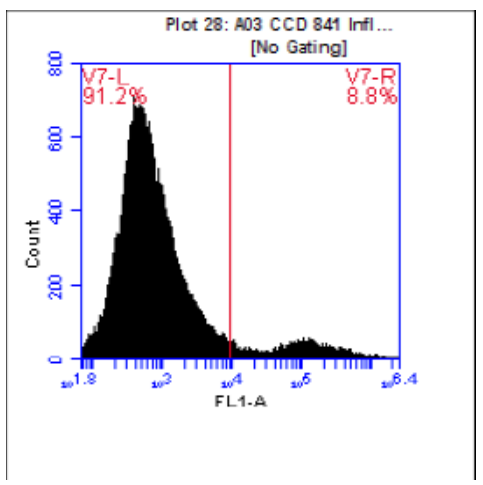

D.

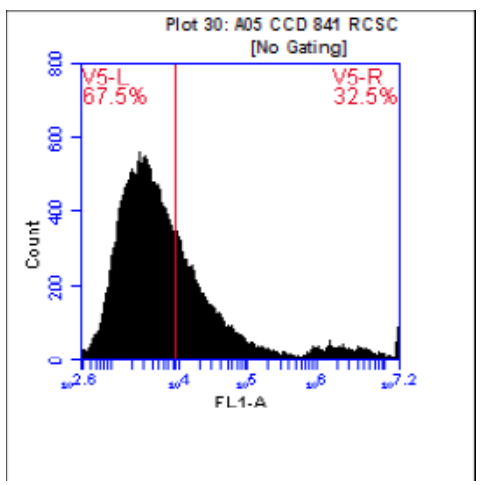

E.

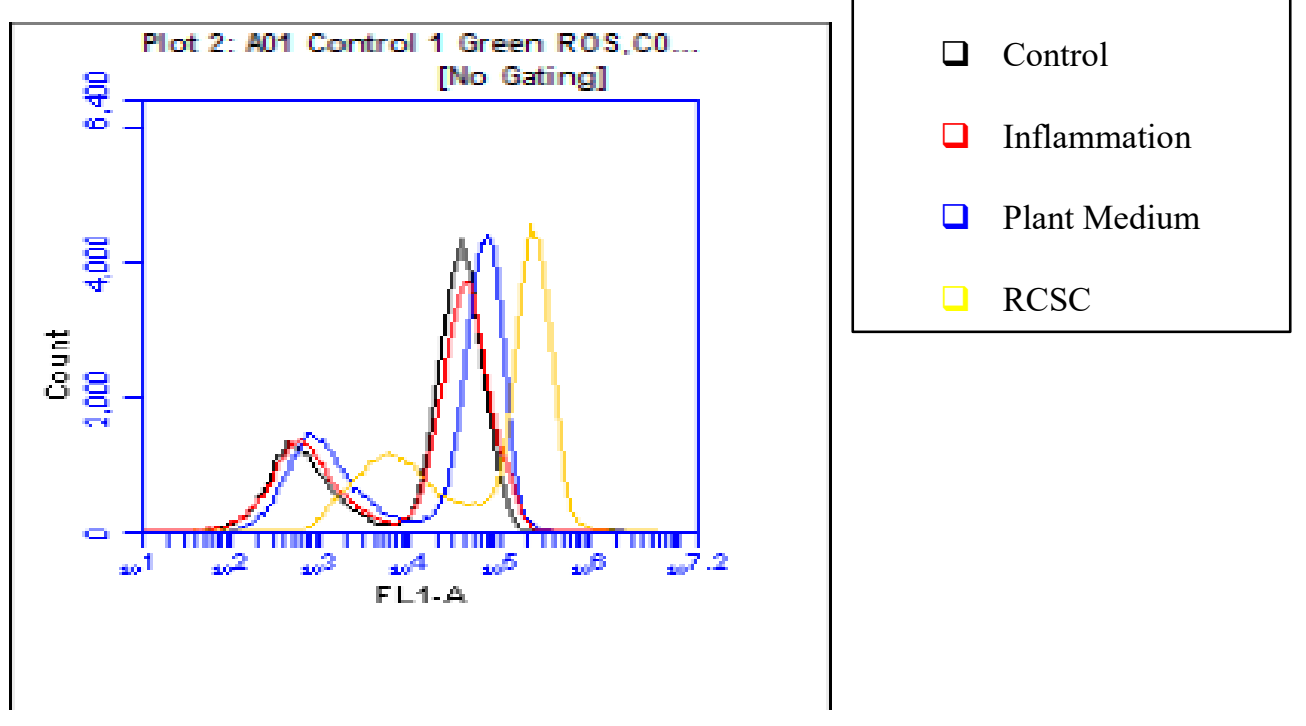


Figure 1.17: RCSC increases nuclear ROS in CCD 841 CoN cell line as analyzed by flow cytometry. CCD $841 \mathrm{CoN}$ cells were analyzed for ROS with CellROX ${ }^{\circledR}$ green nuclear stain following 24 hour treatment with the pro inflammatory cytokine cocktail and an additional 48 hours with a 1:40 diulution of RCSC or plant medium. (A) Control (B) Inflammatory control (C) 1:40 Plant medium control and (D) 1:40 RCSC (E) Merged A-D. Cells were analyzed using an BD Accuri ${ }^{\mathrm{TM}}$ C6 flow cytometer.

\section{RCSC CCD 841 Nuc ROS}

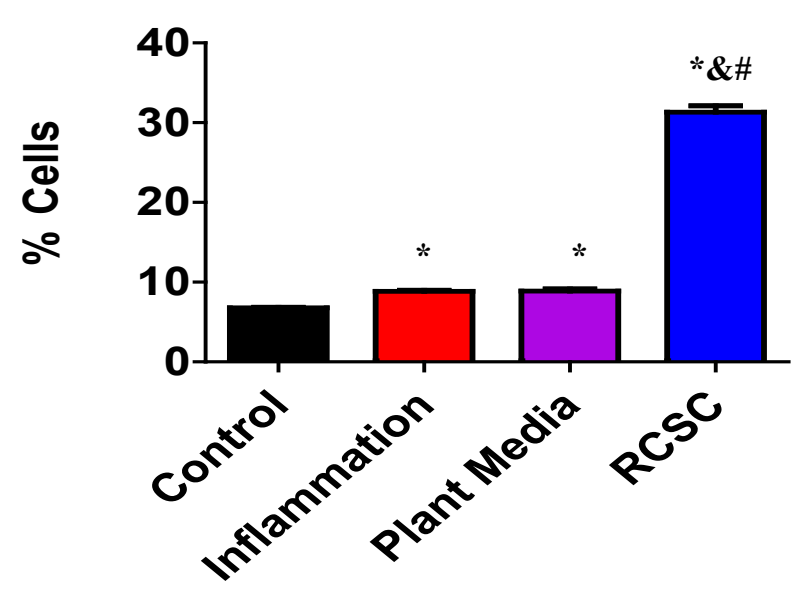

Figure 1.18: Summary data of RCSC nuclear ROS in CCD 841 CoN. Quantification of CellROX ${ }^{\circledR}$ green nuclear ROS staining on $\operatorname{CCD} 841 \mathrm{CoN}$ cell line. $*=$ indicates significance vs. non treated control, $\&=$ indicates significance vs. inflammatory control, \#= indicates significance vs plant medium control. Results analyzed utilizing one way ANOVA with Tukeys test $(\mathrm{p}<0.05)$. 
A.

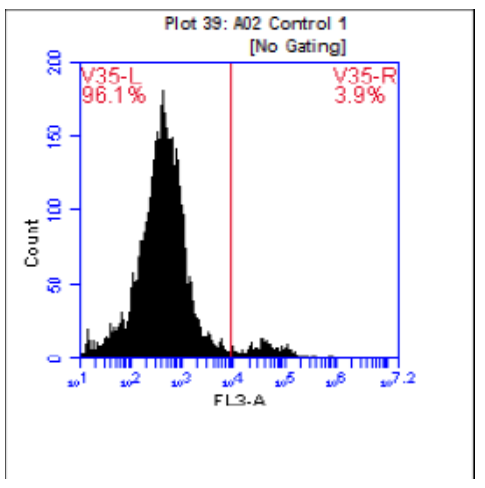

C.

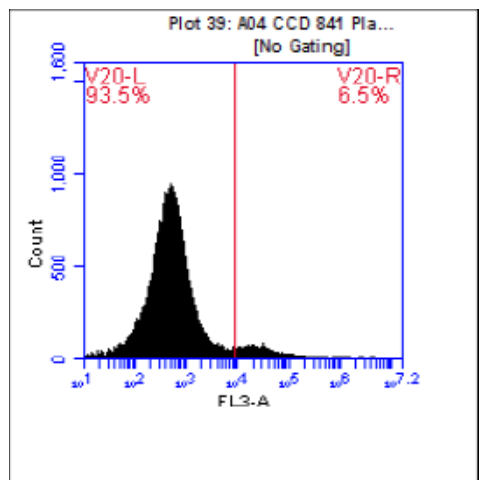

B.

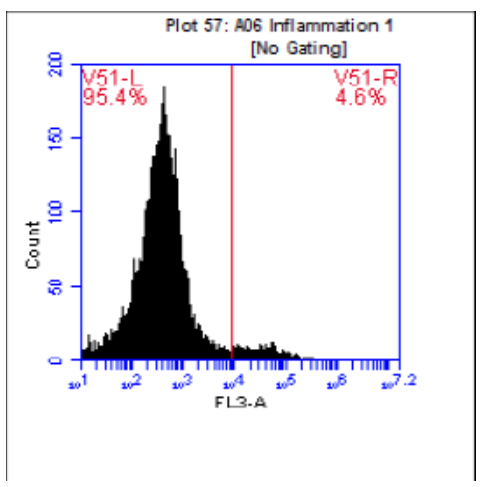

D.

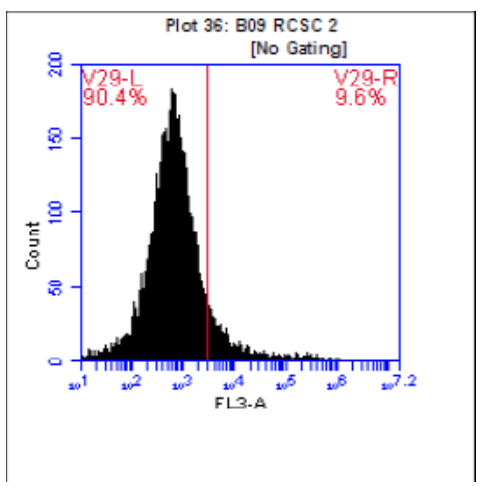

Figure 1.19: RCSC mildly increases cytoplasmic ROS in CCD 841 CoN cell line as analyzed by flow cytometry. CCD $841 \mathrm{CoN}$ were analyzed for ROS with CellROX® orange cytoplasmic stain following 24 hour treatment with the pro inflammatory cytokine cocktail and an additional 48 hours with a 1:40 diulution of RCSC or plant medium. (A) Control (B) Inflammatory control (C) 1:40 Plant medium control and (D) 1:40 RCSC. Cells were analyzed using an BD Accurit ${ }^{\mathrm{TM}}$ C6 flow cytometer. 


\section{CCD 841 CellRox Orange}

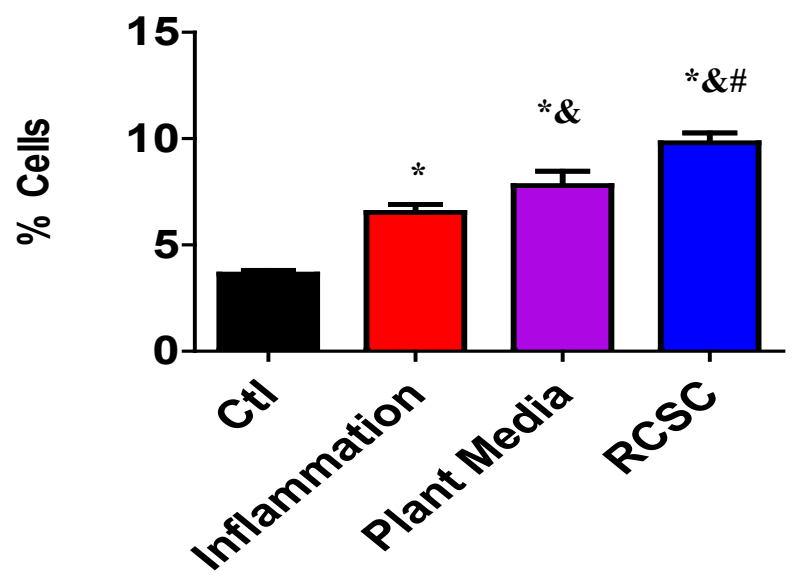

Figure 1.20: Summary data of RCSC cytoplasmic ROS in CCD 841 CoN cell line for flow cytometry. Quantification of CellROX ${ }^{\circledR}$ orange cytoplasmic ROS staining on CCD $841 \mathrm{CoN}$ cell line. $*=$ indicates significance vs. non treated control, $\&=$ indicates significance vs. inflammatory control, \#= indicates significance vs plant medium control. Results analyzed utilizing one way ANOVA with Tukeys test $(\mathrm{p}<0.05)$.

\subsubsection{RCSC treatment reduced the effect of inflammation on cell death in NCM 356 and CCD 841 CoN}

NCM 356 treated with the pro-inflammatory cytokine cocktail for 24 hours followed by 48-hour treatment of RCSC at 1:40 dilution showed significant increase in cell viability following staining with propidium iodide, when the samples were analyzed with FL3 (Figure 1.21 and 1.22, $\mathrm{p}<0.05$ ). Control revealed 96.9\% viable cells, compared with $24.9 \%$ and $16.4 \%$ in inflammatory and PM controls, respectively, followed by $54.6 \%$ viable cells in RCSC treated group. This is suggestive of RCSC reducing cytokine 
induced cell death. Following the same conditions as above, for CCD $841 \mathrm{CoN}$, RCSC increased cell survivability to $89.5 \%$ versus inflammation and plant medium at $79.5 \%$ and $85.5 \%$, respectively (Figure 1.23 and 1.24 ).

A.

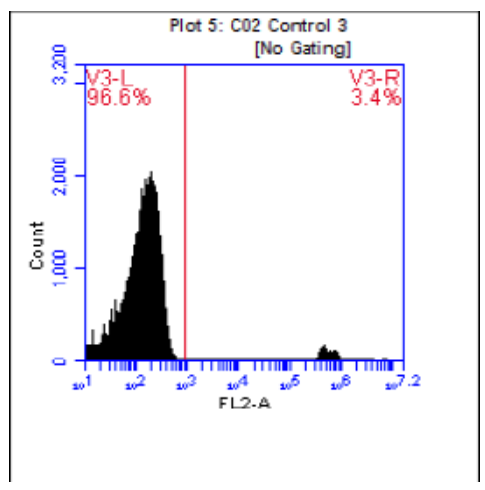

C.

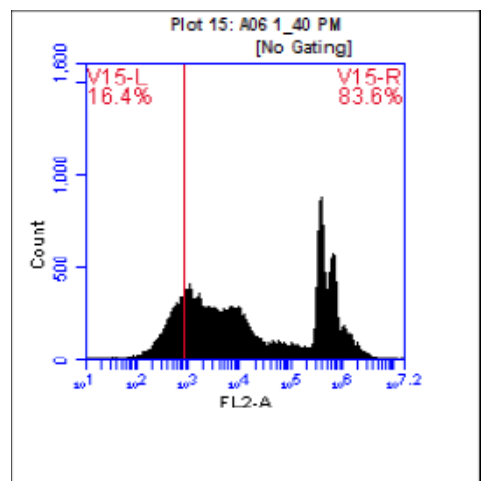

B.

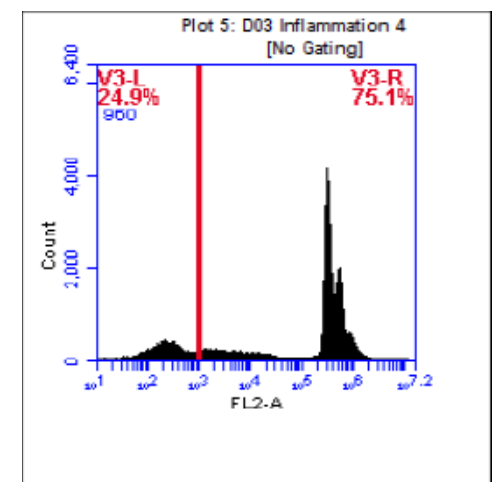

D.

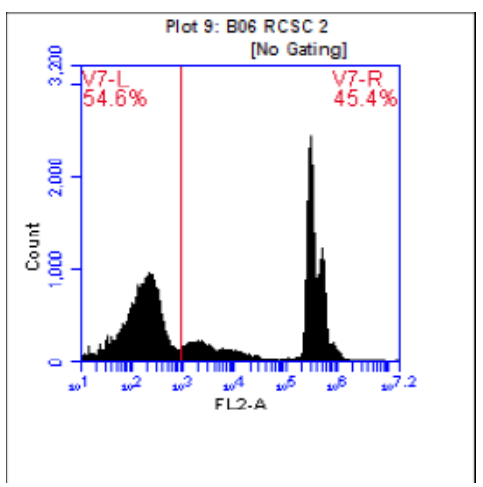

Figure 1.21: RCSC reduced the effect of the pro-inflammatory cytokine cocktail on cell death in NCM 356 cells as analyzed by flow cytometry. NCM 356 cells were analyzed for cell death using propidium iodide cell viability assay following 24-hour treatment with the pro-inflammatory cytokine cocktail and 48 hours of a 1:40 dilution of RCSC or plant medium. Results analyzed utilizing one-way ANOVA with Tukeys test, $\mathrm{p}<0.05$. Analyzed under fluorescent laser three (FL3, 670nm filter). 


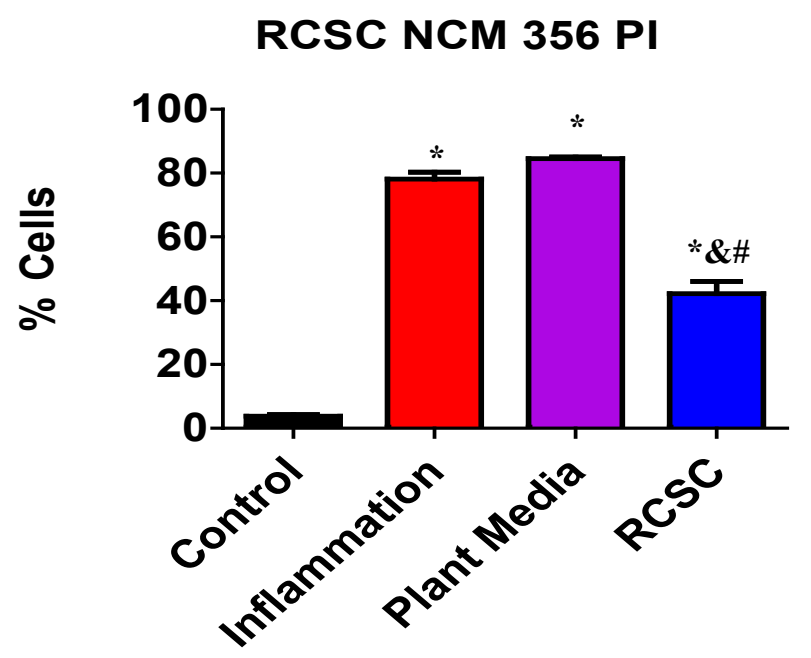

Figure 1.22: Summary data of reduced cell death by RCSC in NCM 356 cell line Quantification of propidium iodide cell viability assay on NCM 356 cells. *= indicates significance vs. non treated control, $\&=$ indicates significance vs. inflammatory control, \#= indicates significance vs plant medium control. Results analyzed utilizing one way ANOVA with Tukeys test $(\mathrm{p}<0.05)$.

A.

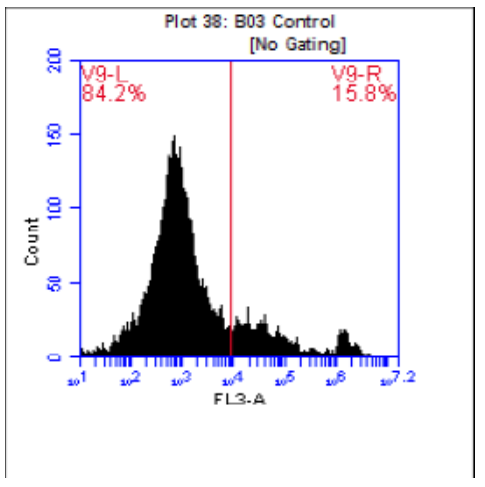

B.

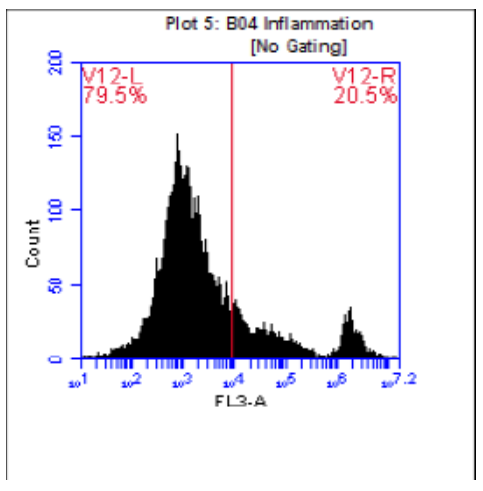


C.

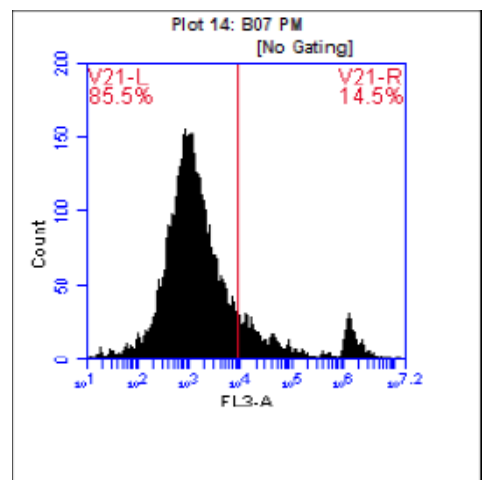

D.

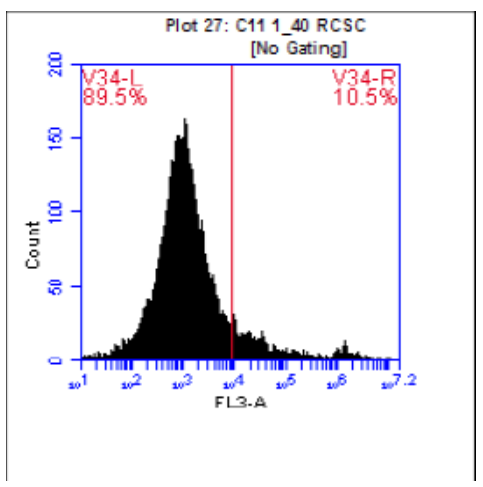

Figure 1.23: RCSC reduced the effect of the pro-inflammatory cytokine cocktail on cell death in CCD 841 CoN cells as analyzed by flow cytometry. CCD 841 CoN cells were analyzed for cell death using propidium iodide cell viability assay following 24hour treatment with the pro-inflammatory cytokine cocktail and 48 hours of a 1:40 dilution of RCSC or plant medium. Results analyzed utilizing one-way ANOVA with Tukeys test, $\mathrm{p}<0.05$. Analyzed under fluorescent laser three (FL3, 670nm filter).

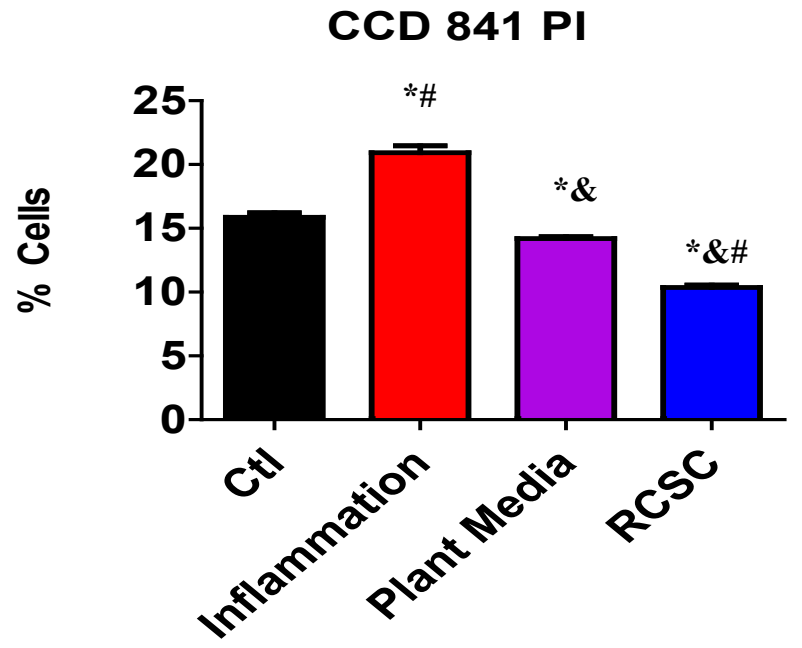

Figure 1.24: Summary data of reduced cell death by RCSC in CCD 841 CoN. Quantification of propidium iodide cell viability assay on CCD $841 \mathrm{CoN}$ cells. *= 
indicates significance vs. non treated control, $\&=$ indicates significance vs. inflammatory control, \#= indicates significance vs plant medium control. Results analyzed utilizing one way ANOVA with Tukeys test $(\mathrm{p}<0.05)$.

\subsubsection{Genes differentially regulated with RCSC treatment identified by quantitative real-time PCR}

Several changes in gene expression (Figure 1.25) were observed in NCM 356 following treatment with the pro-inflammatory cytokine cocktail and RCSC. Five genes

were upregulated two-fold and ten genes were downregulated $\geq$ two-fold. Cluster of differentiation 38 (CD38) was downregulated four-fold, while the cytokine interleukin 2 (IL2) was downregulated 63-fold. Additionally, two genes, nuclear factor kappa-lightchain- enhancer of activated B cells (NF- $\mathrm{kB}$ ) and the oncogene (SKI) were independently upregulated in RCSC versus inflammation and plant medium controls (Table 1.2). Furthermore, four genes, C-C motif ligand 5 (RANTES/CCL5), human leukocyte antigen 1 (HLA1), and inhibitor of kappa light polypeptide gene enhancer in B-cells, kinase beta (IKBKB) were downregulated 2-fold and CD38 was downregulated 4-fold in RCSC versus inflammation and plant medium controls (Table 1.1). 

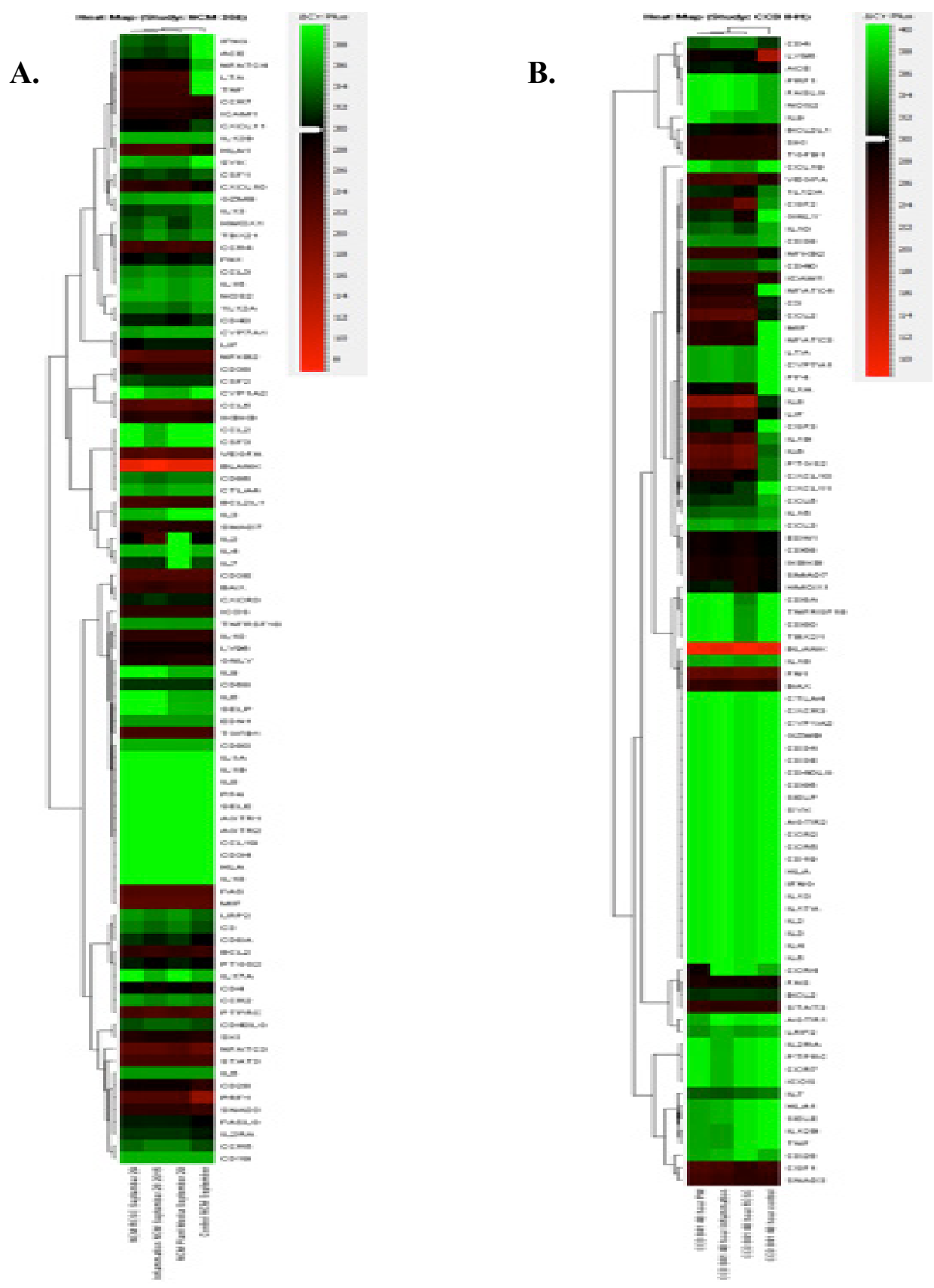

Figure 1.25: Heap map evaluating expression of selected immune responsive genes.

A. NCM 356 treated for 24 hours with the pro-inflammatory cytokine cocktail, followed by 48 hour treatment with RCSC or PM at a 1:40 dilution. B. CCD $841 \mathrm{CoN}$ treated for 24 hours with the pro-inflammatory cytokine cocktail, again followed by 48-hour 
treatment with RCSC or PM at a 1:40 dilution. $\Delta \mathrm{Ct}$ was compared with untreated controls in both groups. Intensity of red or green color indicates up regulation and downregulation respectively. Map generated utilizing Pearson correlation.

Table 1.1: Differentially regulated genes in RCSC vs. inflammatory control in NCM 356

\begin{tabular}{|c|c|}
\hline \multicolumn{2}{|l|}{ NCM 356} \\
\hline Upregulated & Fold Change \\
\hline BAX & $\uparrow^{2}$ \\
\hline CCR4 & $\uparrow^{2}$ \\
\hline CXCL10 & $\uparrow^{2}$ \\
\hline SKI & $\uparrow^{2}$ \\
\hline NF-KB & $\uparrow^{2}$ \\
\hline \multicolumn{2}{|c|}{ Downregulated } \\
\hline $\mathrm{BCL2}$ & $\downarrow_{2}$ \\
\hline CCL5 & $\downarrow_{2}$ \\
\hline CD38 & $\downarrow_{4}$ \\
\hline CD4 & $\downarrow_{2}$ \\
\hline HLA1 & $\downarrow_{2}$ \\
\hline IKBKB & $\downarrow_{2}$ \\
\hline IL -2 & $\downarrow_{63}$ \\
\hline NFATC4 & $\downarrow_{2}$ \\
\hline PTPRC & $\downarrow_{2}$ \\
\hline VEGFA & $\downarrow_{2}$ \\
\hline
\end{tabular}

Table 1.2: Differentially regulated genes in RCSC vs. plant medium control in NCM 356

\begin{tabular}{|ll|}
\hline NCM 356 & \\
\hline Upregulated & Fold Change \\
\hline NF_KB & $\boldsymbol{\uparrow} 2$ \\
\hline SKI & $\boldsymbol{\uparrow} 2$ \\
\hline Downregulated & \\
\hline CCL5 & $\boldsymbol{\downarrow}_{2}$ \\
\hline CD38 & $\boldsymbol{\downarrow}_{4}$ \\
\hline HLA1 & $\boldsymbol{\downarrow}_{2}$ \\
\hline IKBKB & $\boldsymbol{\downarrow}_{2}$ \\
\hline
\end{tabular}

CCD $841 \mathrm{CoN}$ gene expression profile was also investigated in order to identify possible molecular mechanisms. In RCSC versus inflammatory control groups, four genes, interleukin 1 alpha (IL-1A), granulysin (GNLY), colony-stimulating factor 2 (CSF-2), and heme oxygenase decycling 1 (HMOX-1) were upregulated $\geq 4$-fold (Table 1.3). Conversely, 11 genes were downregulated two-fold in RCSC versus inflammatory control, with four genes being downregulated $\geq 4$-fold which include B-cell lymphoma 2 
like 1/BCL2L1, SMAD family member 3/SMAD3, colony stimulating factor 1/CSF1, and C-X-C motif chemokine 10/CXCL10 (Table 1.3).

Table 1.3: Differentially regulated genes in RCSC

vs. inflammatory control in CCD $841 \mathrm{CoN}$

\begin{tabular}{|c|c|}
\hline \multicolumn{2}{|l|}{ CCD $341 \mathrm{CoN}$} \\
\hline Upregulated & Fold Change \\
\hline $\mathrm{CSF}_{2} 2$ & ps \\
\hline GNLT' & T4 \\
\hline HMOK1 & ps \\
\hline$\| \mathrm{L}-1 . \mathrm{A}$ & 14 \\
\hline$\| \mathrm{L}-1 \mathrm{~B}$ & +2 \\
\hline$\| \mathrm{L}-6$ & 12 \\
\hline$\| \mathrm{L}-8$ & $T^{2}$ \\
\hline ㄴ.JF & 12 \\
\hline PTGS2 & +2 \\
\hline \multicolumn{2}{|c|}{ Downregulated } \\
\hline BCL2L1. & 44 \\
\hline $\mathrm{Cs}$ & 42 \\
\hline $\operatorname{cel} 2$ & 42 \\
\hline $\mathrm{CSF}_{-1}$ & 48 \\
\hline CxcL10 & 43 \\
\hline FAS & 42 \\
\hline ICAMI & 42 \\
\hline LY96 & 42 \\
\hline MFATCS & 42 \\
\hline NFATCA & 42 \\
\hline NF_KE & 42 \\
\hline SkI & 42 \\
\hline SMADS & 44 \\
\hline STAT3 & 42 \\
\hline TGFE1 & 42 \\
\hline
\end{tabular}

Likewise, eight genes were upregulated in RCSC versus both inflammatory and plant medium controls, while six genes were downregulated. Of these, three genes, IL1A, GNLY, and CSF-2 were upregulated $\geq 4$-fold (Table 1.4). On the other hand, three genes, SMAD3, CSF-1, and CXCL10 were $\geq 4$-fold were downregulated. Three genes, CXCL10, SKI, and NF-kB were differentially regulated in both NCM 356 and CCD 841 
$\mathrm{CoN}$ in RCSC treated versus inflammatory control group. All three genes were upregulated in NCM 356 while they were downregulated in CCD 841 CoN (Table 1.5).

Table 1.4: Differentially regulated genes in RCSC

vs. inflammation and PM controls

\begin{tabular}{|c|c|}
\hline \multicolumn{2}{|l|}{ CCD $841 \mathrm{CoN}$} \\
\hline Upregulated & Fold Change \\
\hline $\mathrm{CSF}_{-2} 2$ & $\uparrow 8$ \\
\hline GNLY & $\uparrow^{4}$ \\
\hline IL-1A & $\uparrow^{4}$ \\
\hline IL_1B & $\uparrow^{2}$ \\
\hline IL_6 & $\uparrow^{2}$ \\
\hline IL_8 & $\uparrow 2$ \\
\hline LIF & $\uparrow^{2}$ \\
\hline PTGS2 & $\uparrow 2$ \\
\hline \multicolumn{2}{|c|}{ Downregualted } \\
\hline $\mathrm{CSF}_{-1}$ & $\downarrow_{8}$ \\
\hline CXCL10 & $\downarrow_{8}$ \\
\hline FAS & $\boldsymbol{\Downarrow}_{2}$ \\
\hline LY96 & $\downarrow_{2}$ \\
\hline NFATC3 & $\boldsymbol{\Downarrow}_{2}$ \\
\hline SMAD3 & $\downarrow_{4}$ \\
\hline
\end{tabular}

Table 1.5: Differentially regulated genes in NCM356 and CCD 841 CoN when treated with RCSC versus both inflammation and PM controls

\begin{tabular}{|lll|}
\hline Gene & NCM 356 Fold Change & CCD 841 CoN Fold Change \\
\hline CXCL10 & $\uparrow 2$ & $\boldsymbol{\Downarrow}_{8}$ \\
SKI & $\uparrow 2$ & $\boldsymbol{\downarrow}_{2}$ \\
NF-KB & $\uparrow 2$ & $\boldsymbol{\downarrow}_{2}$ \\
\hline
\end{tabular}




\subsection{Discussion}

Maintenance of ROS generation and elimination is a critical mechanism in maintaining cellular homeostasis ${ }^{36}$. High oxidative stress has been shown to play a critical role in a multitude of diseases such as aging, cancer, and neurodegeneration ${ }^{36,37}$. ROS is produced from the reduction of molecular oxygen and includes radical species such as the hydroxyl radical and superoxide. ROS regulate cell survival in a dose dependent manner with moderate levels promoting cell proliferation and survival while high levels disrupting the required homeostasis ${ }^{36}$. Additionally, levels of ROS regulate a multitude of proteins required for cell adhesion, such as cell adhesion molecules (CAMS), which include selectins, integrins and some members of immunoglobulin superfamily ${ }^{37}$

There are two main mechanisms of cell death, notably apoptosis and necrosis. Apoptosis has been characterized as programed cell death dependent on the execution of a family of cysteine dependent aspartate directed proteases, also known as caspases ${ }^{38}$. On the other hand, necrosis has been described as a spontaneous, accidental, uncontrollable process highly dependent on the homeostasis of calcium ${ }^{39}$. Both apoptosis and necrosis are present in a wide range of pathologies from ischemia/reperfusion injuries, to neurodegeneration and inflammation ${ }^{38,39}$. In order to analyze the effects of the proinflammatory cytokine cocktail on apoptotic cell death, propidium iodide was analyzed via fluorescent microscopy and flow cytometer.

InEpC treated with RCSC showed significant decrease in cytoplasmic ROS, while no change was observed in regards to nuclear ROS levels. The greatest reduction in ROS 
was observed in InEpC, when compared to both CCD $841 \mathrm{CoN}$ and NCM 356. Although inflamed NCM 356 cells treated with RCSC showed higher nuclear ROS at 1:40 dilution, following 48 hours incubation, their overall survival increased. Since NCM 356 inappropriately expresses gastrin, and secretes functional gastrin peptides, this could be indicative of a pre-malignant state ${ }^{40}$. Because of the possible pre-malignant state of NCM 356, this could also explain the increase in nuclear ROS. Higher levels of ROS have been correlated with increase in nuclear damage, because of the high reactivity of the free radical. ROS also acts as a transcriptional regulator mainly through interactions with NF$\kappa B$. Treatment with RCSC revealed two fold increase in NF- $\mathrm{kB}$ expression versus all controls, possibly indicative of regulated signaling ${ }^{36}$. Also in both inflammatory and plant medium controls, IKBKB is upregulated two fold, versus RCSC again indicative of regulated signaling outside of canonical signaling which requires IKBKB degradation ${ }^{36}$.

Since RCSC increased nuclear and cytoplasmic ROS and overall survivability increased, this implicates ROS acting in a positive manner. Acting in a positive fashion, ROS could be inhibiting the action of the pro-inflammatory cytokine cocktail. An important point to consider is the concentration of the cytokines (both TNF- $\alpha$ and IL-1 $\beta$ used at $100 \mathrm{ng} / \mathrm{ml}$ ) in the inflammatory cocktail, which is sufficient to directly initiate apoptosis. In RCSC treated samples, the overall number of living cells stained with NucBlue ${ }^{\circledR}$ fluorescent probe was much higher, versus both inflammation and plant medium. Since most cells appeared apoptotic or necrotic in inflammation and plant medium, cells treated with RCSC revealed drastic increase in nuclear generated ROS with minor increase in cytoplasmic ROS. Thus, increase in both nuclear and cytoplasmic 
generated ROS by RCSC, could be the mechanism, which prevents cytokine induced cell death. TNF- $\alpha$ induced inflammation has been shown to induce ROS production through NADPH dependent oxidases ${ }^{41}$. This along with sustained JNK activation, a direct target of TNF- $\alpha$, induces necrosis across multiple cell lines ${ }^{41}$. When NCM 356 was treated with the inflammatory cocktail and RCSC, NF- $\mathrm{KB}$ and BCL2L1 expression increased 2-fold in treated groups, which could account for the increase in survivability since NF-kB activation prevents both apoptosis and sustained JNK activation at normal levels, while BCL2L1 prevents TNF- $\alpha$ activation.

The inflammatory and plant medium control groups both showed increase in nuclear ROS, which could be attributed to treatment with TNF- $\alpha$ as well. Increase in ROS production can hinder the function of MAPK phosphatase (MKP) by altering a critical cysteine residue through oxidation ${ }^{42}$. This impinges MKP function while rendering JNK constitutively active, thus causing cell death as seen in both inflammatory and plant medium controls ${ }^{42}$. IL-2 was upregulated in inflammatory and plant medium control 63-fold and RCSC was able to restore the expression to control levels. Sohn and colleagues have shown that absence of this cytokine can induce colon cancer in a chronic ulcerative colitis setting ${ }^{33}$. IL-2, along with other interleukin class molecules, have also been implicated in regulating the intestinal epithelium through TGF-beta dependent pathways. Therefore, normal regulation of this cytokine is critical in maintaining a healthy population ${ }^{43}$. It has been shown that abnormal increase in cytokines can lead to improper immune responses resulting in inflammation ${ }^{44}$. Mediation of this cytokine as 
well as other by RCSC may be responsible for anti-inflammatory and anti-tumorigenic responses.

CCD $841 \mathrm{CoN}$ showed large increase in nuclear generated reactive oxygen species with mild increase in cytoplasmic generated ROS compared to all controls. This response can possibly be attributed to RCSC having some effect on mitochondrial NADPH oxidases. A major portion of ROS is generated in the mitochondria and is utilized for both cellular defense mechanisms, such as initiating the innate and adaptive immune response as well as cell signaling ${ }^{45}$. In terms of anti-inflammatory effects, the pro-inflammatory cytokine, interferon, stimulated interferon gamma induced protein 10 (CXCL10) 8-fold in both inflammatory and PM controls of CCD $841 \mathrm{CoN}$, whereas RCSC was able to reduce the expression back to control levels. This is a direct response related to the interactions of the pro-inflammatory cytokine cocktail ${ }^{46}$. Furthermore, this chemokine activates the signal transduction pathway involving the constitutively expressed Janus family kinases, Jak, Tyk2, and Stat1, 2, 3 and $5^{47}$. Activation of this pathway has shown to be highly upregulated in inflammatory bowel disease. Treatment with RCSC was shown to further reduce the expression of STAT3, a strong oncogenic transcription factor by two-fold, indicating its immune-modulatory and anti-inflammatory effects.

The pro-inflammatory cytokine TNF- $\alpha$ has been extensively studied in IBD, and has shown to be greatly upregulated, with concentration dependent on disease progression $^{48,49}$. Almost all known members of the TNF family form trimers that interact in a multitude of ways, from initiating a strong inflammatory response, to initiating cell 
death. Most notably, strong interactions of TNF family with FAS work to induce cell death, as well as to initiate strong immune modulatory effects ${ }^{50}$. In both inflammatory and PM groups, FAS was upregulated two-fold, whereas RCSC was able to mitigate this response. This could be one of the main mechanisms in preventing cytokine induced cell death. Interestingly, IL-6 was upregulated in RCSC treated groups in CCD 841 CoN. It binds to form an active IL-6sIL-6R complex, which in turn activates STAT3 ${ }^{51}$. This response results in colitis, as well as triggering oncogenic event. However, IL-6 alone can prevent apoptosis, which could partially account for increased survivability in RCSC treated groups ${ }^{51}$.

\subsection{Conclusion and future work}

RCSC exhibits strong anti-inflammatory effects following high doses of proinflammatory cytokines. Large increase in ROS suggested protective and immune modulating effect of RCSC. The extent to which RCSC impacts cellular function is dependent on downstream targets such as FAS, NF-кB, STAT3, CXCL10, IL-2, IL-6, as well as others, needed to maintain cellular homeostasis. Interleukin 2 expression increased several-fold following pro inflammatory cytokine treatment, and was reduced to control levels following RCSC possibly indicative of its mechanism of action. Additionally, CXCL10 and CSF-1 were downregulated several-fold compared to both inflammation and PM controls, further indicating its mode of action. Further research is needed to fully understand the effects in vivo and in vitro, specifically further elucidation 
of molecular mechanisms, as well as uncovering the underlying metabolite(s) responsible for these effects.

\subsection{Acknowledgements}

This work was supported by the National Institute of Food and Agriculture, U.S. Department of Agriculture, under award number 2014-67018-21767. 
Chapter 2: Isolation of Bioactive Fractions from Rice Callus Suspension Culture (RCSC) 


\subsection{Abstract}

Previous reports on RCSC have indicated strong anti-inflammatory and anticancer activity. We sought to identify the bioactive compounds and/or fractions responsible for such activity. Twenty-five $1 \mathrm{ml}$ fractions were isolated via column chromatography and further analyzed by high performance liquid chromatography (HPLC). A total of 6 peaks were identified in fractions 1-7, whereas fractions 8-25 had 3 peaks. Fractions 10-18 were pooled and re-analyzed by HPLC utilizing an aqueous mobile phase: a total of 8 peaks were identified. Cell viability was assayed with whole RCSC and fractions 10-18 for anticancer activity using the colon adenocarcinoma cell line SW620. Furthermore, reactive oxygen species (ROS) assays were utilized to test for anti-inflammatory activity following treatment with a pro-inflammatory cytokine cocktail. RCSC and fractions 10-18 both significantly reduced the number of viable cells in SW620, while mild decrease was seen in CCD 841 CoN. Additionally, RCSC fractions greatly increased nuclear and cytoplasmic ROS as analyzed by flow cytometry versus both untreated and inflammatory control. In conclusion, RCSC fractions 10-18 consists of compounds that are responsible for the anticancer, anti-inflammatory and immune regulating properties as previously reported. 


\subsection{Introduction}

Natural products are the most important source of medicinal drugs today ${ }^{52}$. World Health Organization (WHO) back in 1985 estimated that about 65\% of the world population relied on plant derived medicinals ${ }^{3}$. Today, a plethora of these compounds are used to treat a wide variety of ailments. Eucalyptol for example, along with camphor, which are found readily in rosemary oil, have shown to contain anti-inflammatory and anti-cancerous activity $^{52}$. The diterpenes, nigilactones, have shown anti-leukemic activity $^{52}$. The anti-mitotic paclitaxel is used extensively to treat breast cancer ${ }^{52}$. Several flavones isolated from a variety of plant species have shown efficacy across the board. The compound acacetin, isolated from black locust, exhibits anti-inflammatory activity ${ }^{52}$. Furthermore, several flavanone derivatives show biological activity. The silymarins, present in milk thistle, are effective agents against liver damage as well as useful in prevention of skin cancer ${ }^{52}$.

The bioactive compounds of natural products are produced as "secondary metabolites" through four major pathways, malonic acid pathway, mevalonic acid pathway, non-mevalonate pathway, and shikimic acid pathway ${ }^{25,27,53}$. These pathways produce three main classes of compounds with several existing derivations, alkaloids, phenylpropanoids and allied phenolic compounds, and terpenoids $1^{1,22,24,26,28}$.

The list of natural products with medicinal properties is seemingly endless; several reviews, books, dictionaries and databases have been specifically dedicated to the understanding of these compounds ${ }^{52}$. The most challenging aspects of natural products, for use in modern medicine, revolve around isolation and purification. There are several 
factors, which affect the final outcome such as type of plant tissue, solvent used, temperature, pressure and time ${ }^{54}$. Additionally, the extraction method chosen greatly affects the final outcome; therefore there is great need for a standard and integrative $\operatorname{approach}^{53}$.

Several different techniques have been utilized for natural product extraction. There are two main approaches, conventional and non-conventional techniques. The current conventional techniques are soxhlet extraction, maceration and/or hydro distillation $^{53}$. The non-conventional techniques are enzyme-assisted extraction, ultrasound assisted extraction, supercritical fluid extraction, pressurized liquid extraction, and pulsed electric field assisted extraction ${ }^{53}$.

Recently, our group has reported potent anti-cancer and anti-inflammatory activity in rice callus suspension culture (RCSC). Our current aim is to isolate, purify, and characterize the bioactive compound in RCSC utilizing conventional extraction and purification techniques. This includes utilizing solvent-based extractions along with following methods for purification and structural determination: thin layer chromatography (TLC), column chromatography, high performance liquid chromatography (HPLC), and nuclear magnetic resonance spectroscopy (NMR.

Inflammatory bowel disease (IBD) has emerged as a dominant inflammatory condition affecting millions across the globe, 1.2 million in the United States alone. Moreover, IBD drastically impacts morbidity and quality of life for those affected ${ }^{55}$. Colorectal cancer, on the other hand, is the third most commonly diagnosed cancer 
worldwide with around 700000 deaths annually ${ }^{56}$. In this study, we utilized an in vitro IBD model, along with a colorectal adenocarcinoma cell line SW 620 to identify possible bioactive metabolites in RCSC consistent with previous reports ${ }^{12,13}$. We identified and characterized an active fraction in RCSC with strong reactive oxygen species (ROS) modulating affects. Furthermore, the fractions had potent anticancer activity in vitro with minimal affects on the normal colon cell line CCD $841 \mathrm{CoN}$.

\subsection{Materials and methods}

\subsubsection{Rice callus suspension culture}

Rice callus suspension culture was produced from Oryza sativa cultivar Nipponbare seeds from the United States Department of Agriculture (USDA) National Plant Germplasm System (NPGS) following the protocol as described by Deshpande et al. ${ }^{13}$. Seeds were incubated on solid MS medium supplemented with Chu's N6 vitamins (PhytoTechnology Lab) and 2,4-dichlorophenoacetic acid (2,4-D) for 3 weeks at $28^{\circ} \mathrm{C}$. Next, one $10 \mathrm{~mm}$ petri dish of callus was added to $250 \mathrm{ml}$ of liquid callus induction medium and grown for an additional 3 weeks at $28^{\circ} \mathrm{C}$ on a shaker. Following 3 weeks, the liquid medium was collected, particulate matter and cell debris was removed through centrifugation at $10,000 \mathrm{rpm}$ for $10 \mathrm{~min}$ at $4^{\circ} \mathrm{C}$ and filter sterilized through .2 micron filters. The culture was further aseptically aliquoted into $50 \mathrm{ml}$ sterile culture tubes and stored at $-20^{\circ} \mathrm{C}$ until further use. UV absorbance at $250 \mathrm{~nm}-300 \mathrm{~nm}$ was measured via Nanodrop. 


\subsubsection{Preparation of RCSC for phytochemical analysis and thin layer chromatography (TLC)}

$1 \mathrm{ml}$ of RCSC was centri-vaped to remove callus liquid induction medium/water and then re-suspended in $1 \mathrm{ml}$ of $100 \%$ methanol, acetone, and/or ether. Samples were then applied to Thin Layer Chromatography (TLC; silica gel plates) and developed in various organic solvent combinations. Following development, compounds were identified by exposure to $I_{2}$ vapors. Rf values were calculated for each compound.

\subsubsection{Column chromatography}

RCSC was further analyzed and separated via silica column chromatography to test and isolate active fractions. $500 \mathrm{ml}$ of RCSC was concentrated via rotary evaporation to $15 \mathrm{ml}$ to create a $33 \mathrm{x}$ concentrated solution. The solution was again filtered through a .2-micron filter and aseptically aliquoted into $50 \mathrm{ml}$ sterile centrifuge tubes. UV-visible spectrum was analyzed via Nanodrop. A 50ml column was packed with $10-40 \mu \mathrm{m}$ silica gel and soaked with a 20/80-methanol/chloroform solvent system. $1 \mathrm{ml}$ of $33 \mathrm{x}$ concentrated RCSC was added to the column and fractions were collected in $1 \mathrm{ml}$ vials. Next, each $1 \mathrm{ml}$ fraction was spotted on TLC plates and analyzed as described above.

\subsubsection{High Performance Liquid Chromatography (HPLC)}

RCSC was fractionated using high performance liquid chromatography (HPLC). RCSC was concentrated $33 \mathrm{x}(660 \mathrm{mg} / \mathrm{ml})$ as described above. $1 \mathrm{ml}$ was further centrivaped and re-suspended in $100 \%$ methanol and/or sterile deionized water as previously

described $^{12} .100 \mu 1$ of the sample was injected into a C18 reverse phase (Kinetex ${ }^{\mathrm{TM}}$ ) 
and/or PFP column (Kinetex ${ }^{\mathrm{TM}}$ ) and fractionated using a photo diode array detector (Agilent Technologies). Compounds were separated using a binary gradient of 50\% methanol (solvent A) and 50\% acetonitrile (solvent B) and/or 5\% acetic acid (Solvent A) and $50 \%$ acetonitrile (solvent $\mathrm{B})^{12}$. Total run time was 60 minutes.

\subsubsection{Human colon adenocarcinoma cell line SW620 culturing, passaging, and seeding}

The cell line selected for high throughput screening of column chromatography and HPLC fractions was SW620 obtained from ATCC (American Type for Culture Collection). SW620 was cultured in Roswell Park Memorial Institute medium (RPMI1640) supplemented with $10 \%$ Fetal Bovine Serum (FBS) and $1 \%$ penicillin, streptomycin and amphotericin B solution (ATCC). Cells were frozen in 95\% complete growth medium supplemented with 5\% DMSO and stored in liquid nitrogen vapor phase until needed.

Cells were removed from liquid nitrogen and quickly thawed in $37^{\circ} \mathrm{C}$ water bath for no more then 1 minute. Next, cells were immediately placed in $10 \mathrm{ml}$ of complete growth medium and centrifuged to remove residual DMSO. $10 \mathrm{ml}$ of complete growth medium was added to the pellet and gently mixed and then further transferred to $100 \times 20$ $\mathrm{mm}$ sterile culture dish. Cells were incubated at $37^{\circ} \mathrm{C}, 5 \% \mathrm{CO}_{2}, 95 \%$ humidity.

Once cells reached $70-90 \%$ confluence, medium was removed and cells were treated for 5-10 minutes with 3-4ml of trypsin-EDTA solution (Hyclone) to detach the monolayer as per manufacturer recommendations. $7 \mathrm{ml}$ of complete growth medium was 
added to the culture dish to inactivate trypsin-EDTA solution. Then, cells were collected and centrifuged at 1000rpm. The pellet was then re-suspended in complete growth medium and either passaged or seeded at the appropriate density in $6,12,24$, or 96 well plates. Cells were sub-cultured at 1:2-1:10 ratio as per manufacturer recommendations with media renewal 2 to 3 times per week.

Cell counting was done using a hemocytometer and trypan blue cell exclusion assay. Cells were first diluted to a 1:20 ratio with cell culture medium: $25 \mu 1$ of cells added to $475 \mu \mathrm{l}$ of cell culture medium. $50 \mu 1$ of the diluted sample was then added to $10 \mu \mathrm{l}$ of the trypan blue stain. Lastly, $10 \mu \mathrm{l}$ was added to the hemocytometer for cell counting. Cells were counted following the formula as described by Deshpande et al. ${ }^{13}$. Number of cells $/ \mathrm{mL}=(\mathrm{X}) *(20) *(1.2) * 10^{4}$, where $\mathrm{X}=$ average number of cells in four squares, $20=$ first dilution factor, and $1.2=$ second dilution factor.

\subsubsection{Normal human colon cell lines culturing, passaging, and seeding}

CCD $841 \mathrm{CoN}$ (ATCC ${ }^{\circledR}$ CRL-1790 ${ }^{\mathrm{TM}}$ ) was cultured in Eagle's minimum essential medium (EMEM, ATCC $® 30-2003$ ) with $10 \% \mathrm{FBS}$ at $37^{\circ} \mathrm{C}$ with $5 \% \mathrm{CO}_{2}$. Cell dilution and counting was done as described above for SW620. Cells were grown until $70-90 \%$ confluence, and then collected using trypsin EDTA. From there, cells were centrifuged and re-suspended in the appropriate cell culture medium for seeding. Seeding was done in $6,12,24$, and/or 96 wells plates at appropriate density's and incubated for 24 hours at $37^{\circ} \mathrm{C}, 5 \% \mathrm{CO}_{2}$ until further use. 
Cell freezing protocol for CCD 841 CoN was done per manufacturer recommendations. The cell monolayer was detached following a 5-15 min treatment of trypsin-EDTA. From there $6-8 \mathrm{ml}$ of complete culture medium was added to inactivate the trypsin-EDTA solution. The cells were then collected and transferred into 50ml conical centrifuge tubes and centrifuged at $125 \mathrm{~g}$ for 5-10 minutes. The resulting cell pellet was furthermore re-suspended in complete growth medium and 5\% DMSO. A stepwise temperature reduction was utilized for cell freezing. First, the cells were placed in $20^{\circ} \mathrm{C}$ for 24 hours, then $-80^{\circ} \mathrm{C}$ for an additional 24 hours, and finally liquid nitrogen for longterm storage.

\subsubsection{Treatment with pro-inflammatory cytokine cocktail}

In order to stimulate an inflammatory response in vitro, cells were treated with a pro-inflammatory cytokine cocktail consisting of TNF- $\alpha$ (100ng/ml, Sigma), IL-1 $\beta$ (100ng/ml, Sigma), LPS (10 $\mu \mathrm{g} / \mathrm{ml}$, Sigma), and IFN- $\gamma(5 \mu \mathrm{g} / \mathrm{ml}$, Sigma) suspended in PBS, for 0-24 hours prior to RCSC or PM treatment.

\subsection{8 in vitro cytotoxicity testing}

Previous literature has indicated strong anti-cancer activity of RCSC in multiple cell lines ${ }^{12,13}$. Therefore in order to test for bioactivity, SW620 was initially assayed with propidium iodide ReadyProbes ${ }^{\circledR}$ reagent using fluorescent microscopy and flow cytometry following treatment with each fraction. The fractions with like Rf values were pooled and rotary evaporated to remove methanol, then re-suspended in PBS and tested for bioactivity. SW620 was trypsinized and collected. Cells were then seeded in 12 well 
plates and grown until 70-90\% confluent. Cells were treated with a 1:5 dilution of each fraction for $24-48$ hours and incubated at $37^{\circ} \mathrm{C}, 5 \% \mathrm{CO}_{2}, 95 \%$ humidity. Following the incubation period, the medium was removed, cells trypsinized and then collected and resuspended in HEPES based live cell imaging solution. Lastly, cells were treated with propidium iodide and analyzed via flow cytometry or fluorescent microscopy.

Cytotoxicity and inflammatory responses were tested in normal colon cells (CCD $841 \mathrm{CoN})$. Cells were cultured and seeded as per manufacturer recommendation. The cells were then treated for 48 hours with a 1:5 dilution of fractionated RCSC (HPLC fractions 10-18) and analyzed via flow cytometer with propidium iodide ReadyProbes ${ }^{\circledR}$. Furthermore, reactive oxygen species (ROS) assays were utilized to analyze free radical scavenging and anti-inflammatory effects following treatment with a pro-inflammatory cytokine cocktail. Cells were treated with the pro-inflammatory cytokine cocktail for 24 hours prior to 48-hour treatment with a 1:20 dilution of RCSC fraction. Following treatments, cells were analyzed for free radical scavenging capabilities utilizing CellROX ${ }^{\circledR}$ orange cytoplasmic and green nuclear ROS probes via flow cytometer under fluorescent laser 3 and 1 (FL3 and FL1), respectively. Statistical analysis of data was performed with one way ANOVA with Tukey's test $(\mathrm{p}<0.05)$. 


\subsection{Results}

\subsubsection{TLC plates identified spots in RCSC with different Rf values}

Thin layer chromatography was performed with RCSC, and $3 x$ and $33 x$ concentrated RCSC solution (Figure 2.1) in order to determine approximately the number of compounds in RCSC. Different running solvents and ratios were utilized. Distilled water was the initial extraction solvent and methanol was used thereafter. The $\mathrm{Rf}$ values for compounds identified are listed below in Table 2.1.

A.

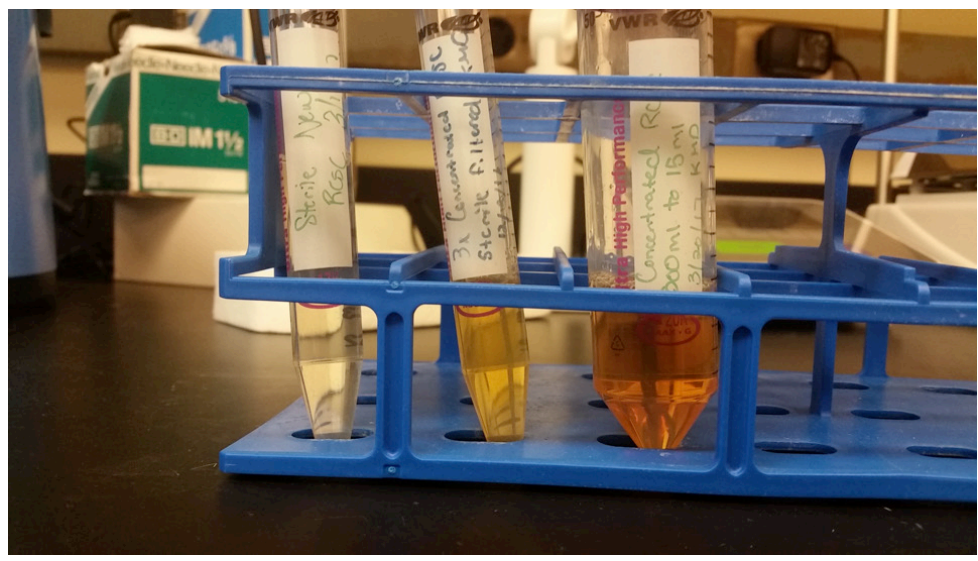


B.

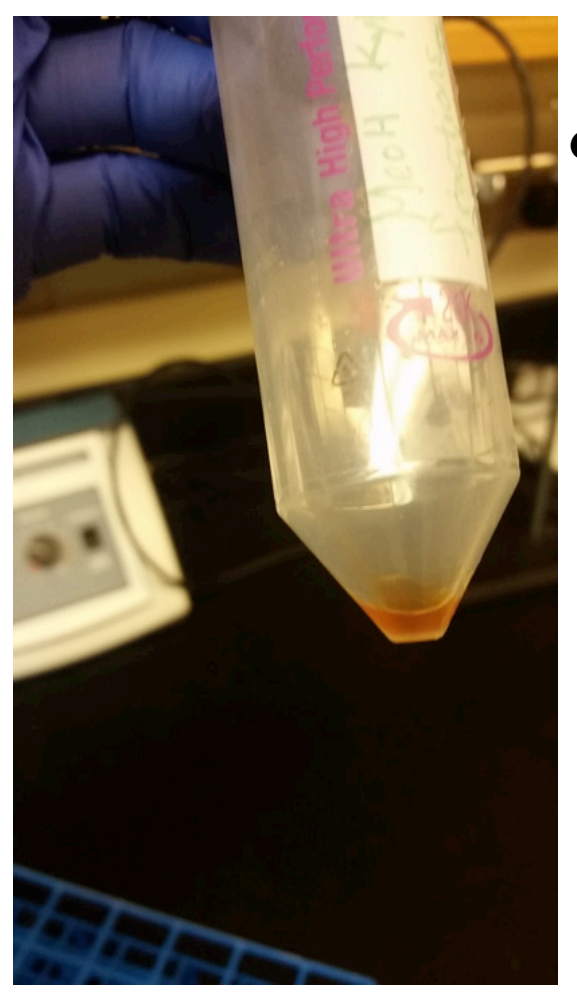

C.

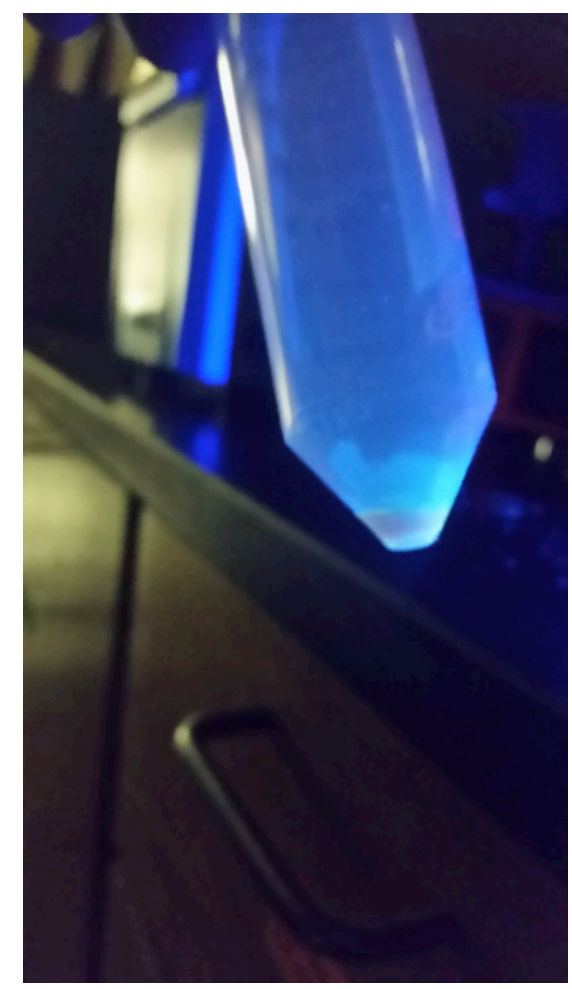

D.

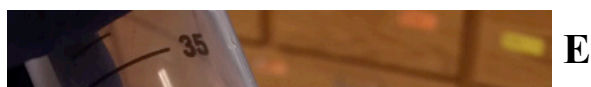

E.

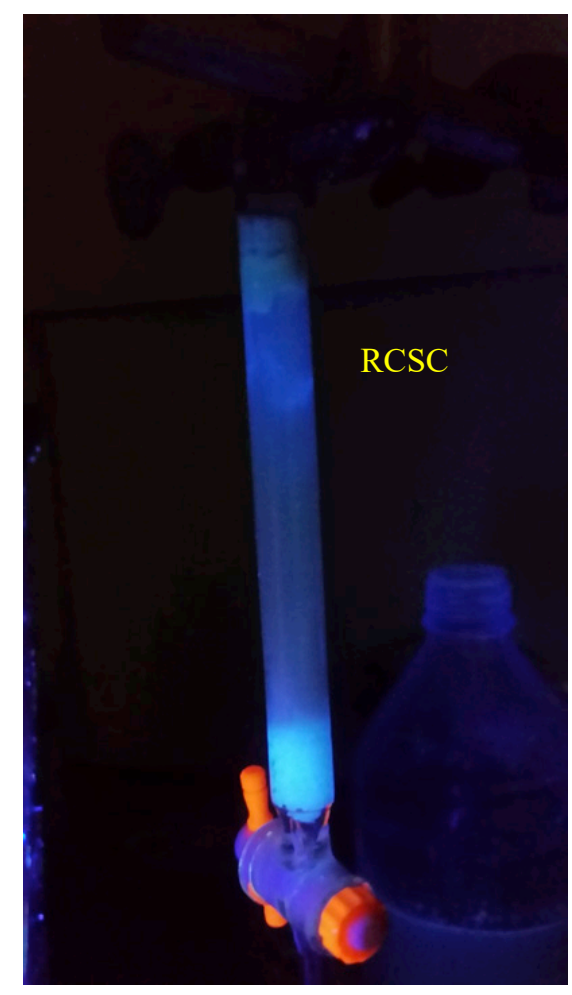


Figure 2.1: Images of RCSC. A. From left to right imaged RCSC, 3x, and 33x concentrated RCSC. B. Methanol extract of concentrated RCSC, which produced an oily sap like substance. C. Methanol extract showing UV activity. D. Methanol extract in $\mathrm{DDH}_{2}$, poor solubitlity. E. Column chromatography of 33x Concentrated RCSC running through silica gel.

Table 2.1 Rf values for compounds identified in RCSC

\begin{tabular}{|c|c|c|c|}
\hline \multirow[t]{4}{*}{ Sample } & Extraction Solvent & Running Solvent & Rf Value \\
\hline & & & Compound $1=.83$ \\
\hline & & & Compound $2=.61$ \\
\hline & & & Compound $3=.19$ \\
\hline & & & Compound $1=.9$ \\
\hline & & & Compound $2=.7$ \\
\hline & & & Compound $1=.93$ \\
\hline & & & Compound $2=.58$ \\
\hline & & & Compound $1=.54$ \\
\hline & & & Compound $2=.33$ \\
\hline $\begin{array}{l}\mathrm{RCSC} \\
3 \mathrm{x} \\
\end{array}$ & Aqueous & $\begin{array}{l}20 / 80 \\
\text { Methanol/Chloroform }\end{array}$ & .93 \\
\hline $\begin{array}{l}\text { RCSC } \\
33 \mathrm{x}\end{array}$ & Aqueous & $\begin{array}{l}\text { 20/80 } \\
\text { Methanol/Chloroform }\end{array}$ & .41 \\
\hline
\end{tabular}




\subsubsection{Fractionation of RCSC with column chromatography and HPLC}

Column chromatography was utilized to separate compounds from the $33 \mathrm{X}$ concentrated RCSC solution. A total of twenty-five $1 \mathrm{ml}$ fractions (Figure 2.2) were collected with varying mobile phases. Fractions 1-7 were collected utilizing a 20/80 methanol/chloroform mobile phase. Fractions $8-25$ were collected via a $100 \%$ methanol mobile phase. Each fraction was further analyzed by HPLC on a C18 column with 50/50 methanol/acetonitrile mobile phase, with UV $255 \mathrm{~nm}$ for compound identification (Figure 1). Fractions 1-7 yielded 6 different peaks, while fractions $8-25$ yielded 3 different peaks. Fractions 10-18 were pooled and further analyzed by HPLC on a C18 column with $100 \%$ HPLC grade water as the mobile phase. A total of 8 peaks were identified, peak 6 was UV excited (Figure 2.3) whose bioactivity has to be tested.

Fraction 1

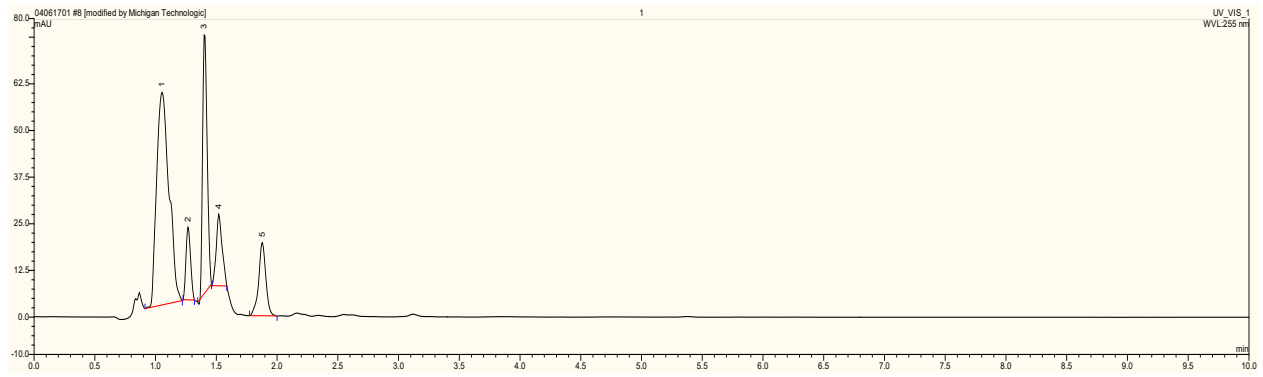

Fraction 2

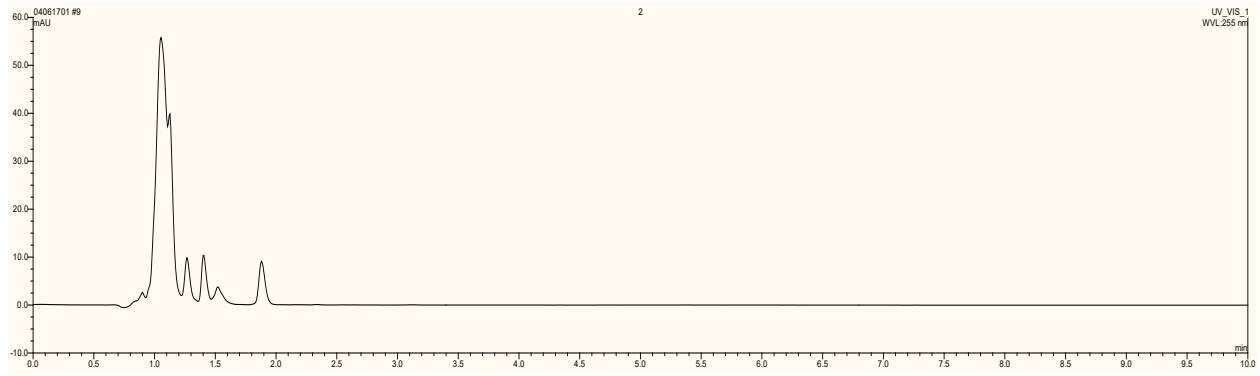


Fraction 3

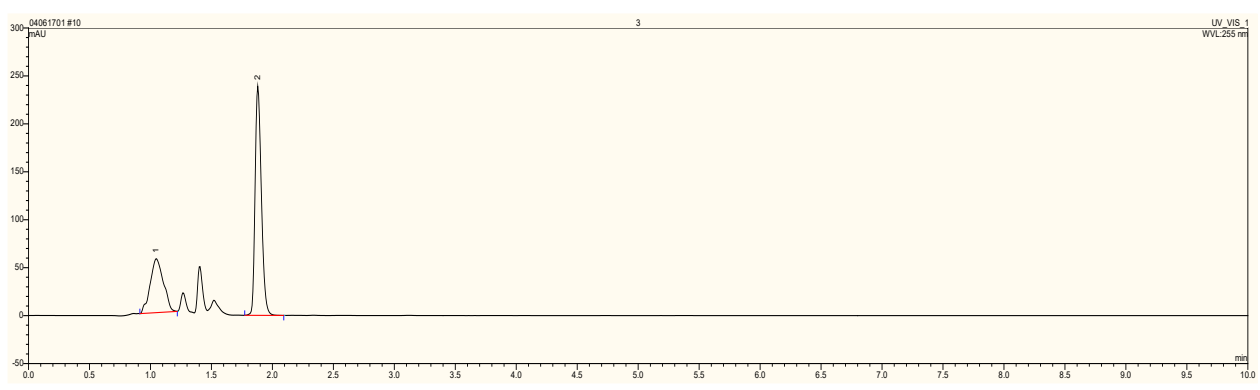

Fraction 4

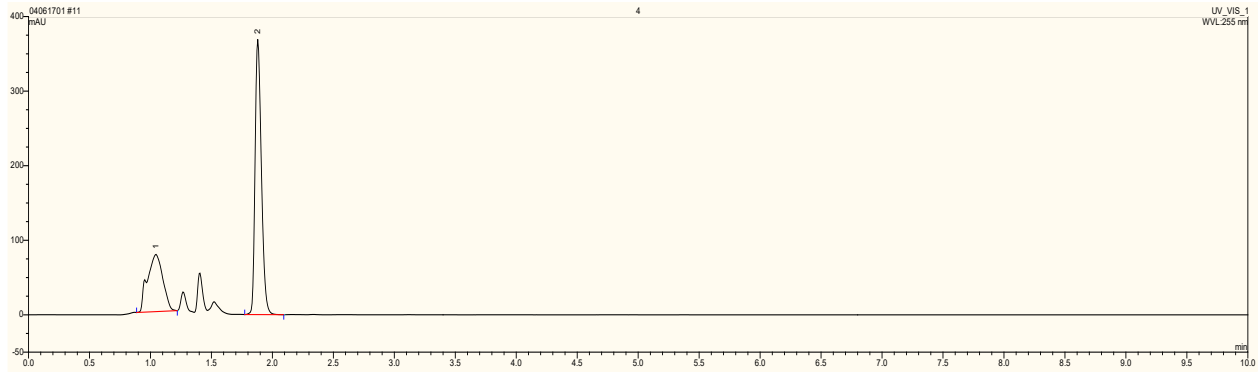

Fraction 7

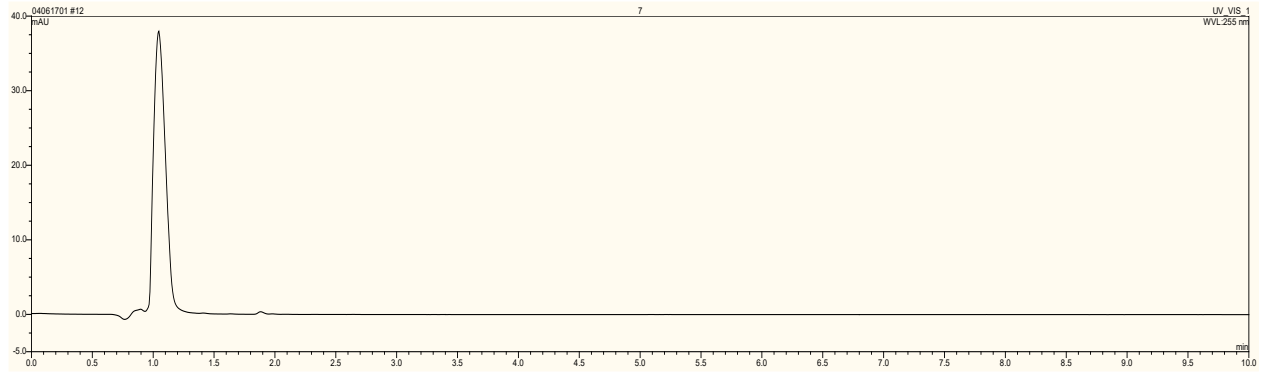

Fraction 8

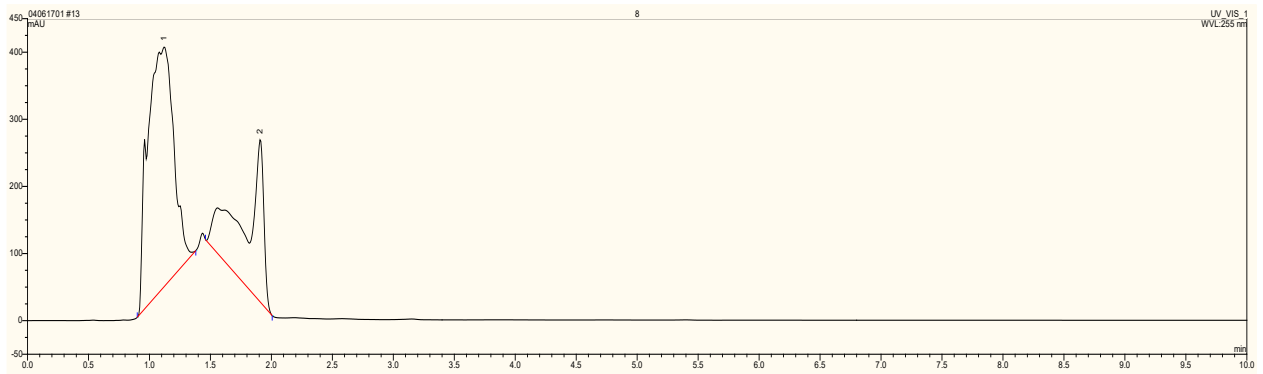


Fraction 9

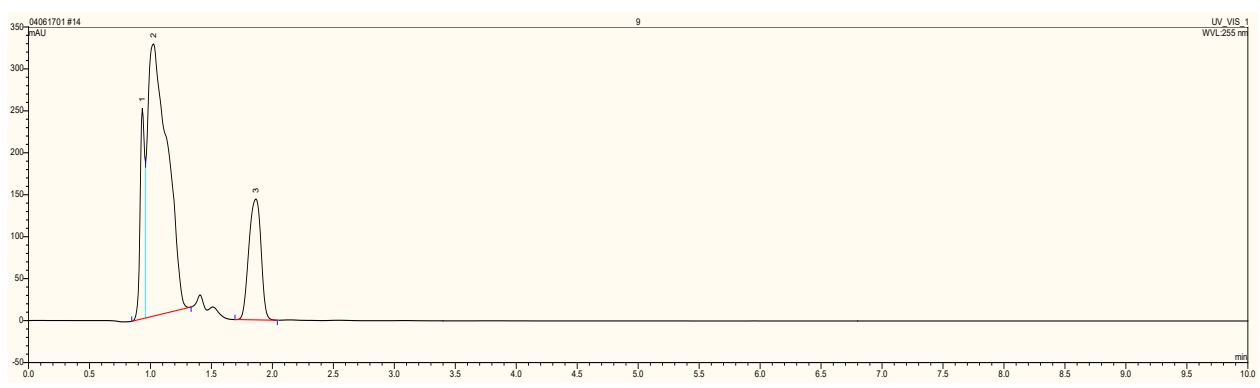

Fraction 10

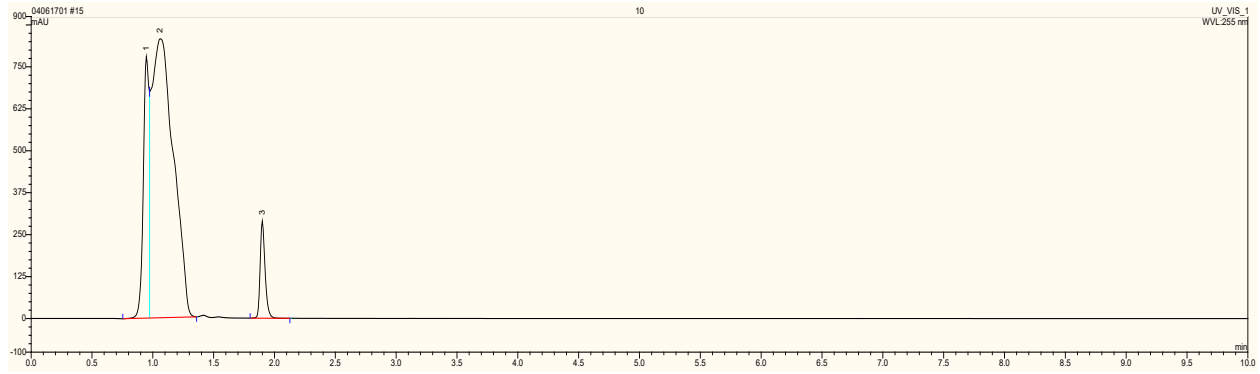

Fraction 11

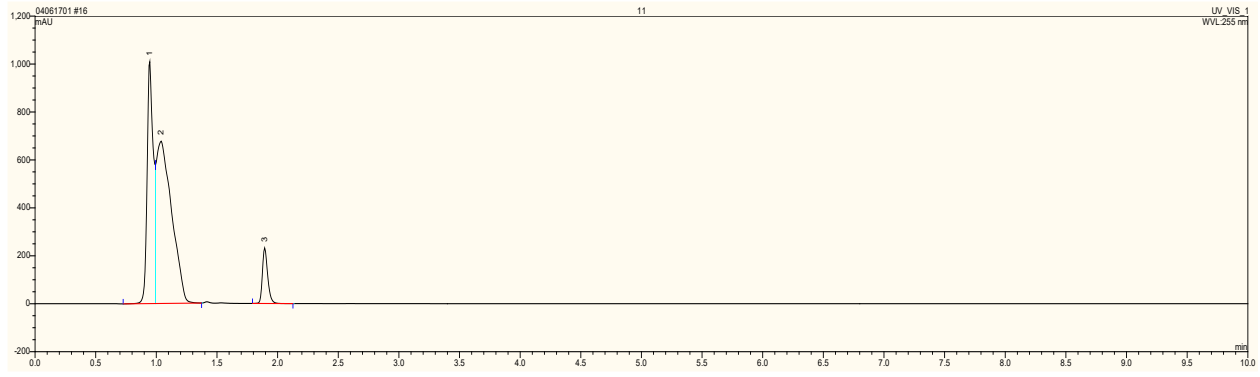

Fraction 12

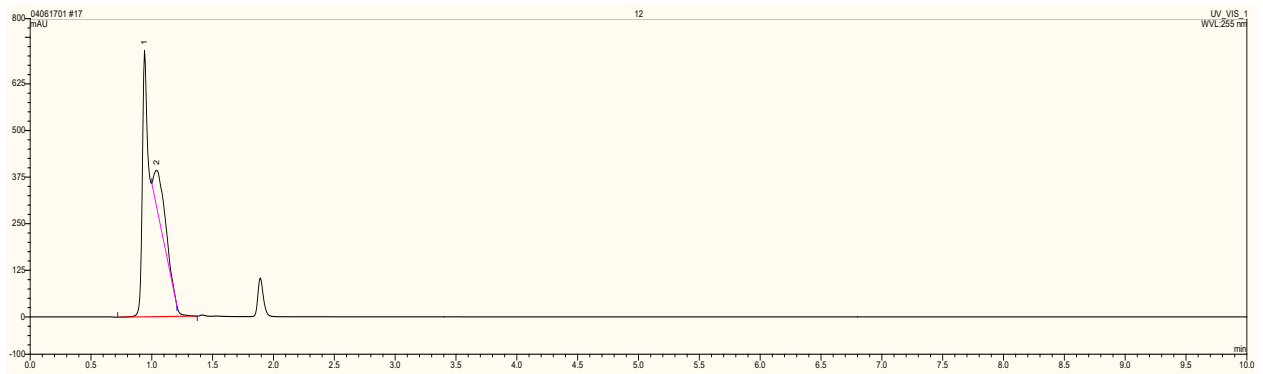


Fraction 13

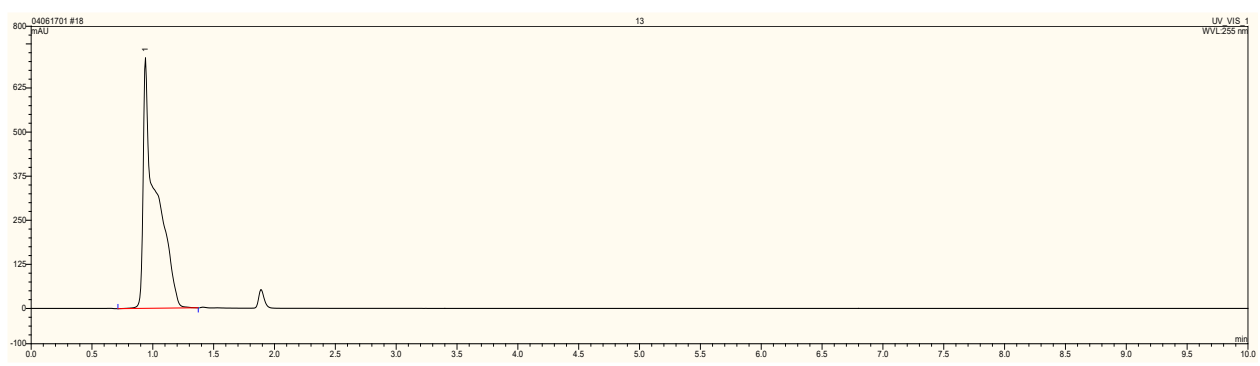

Fraction 14

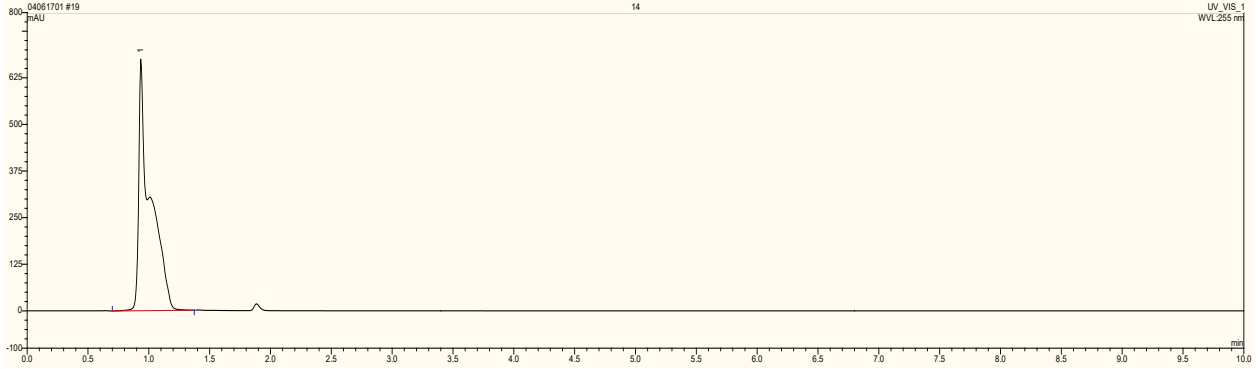

Fraction 15

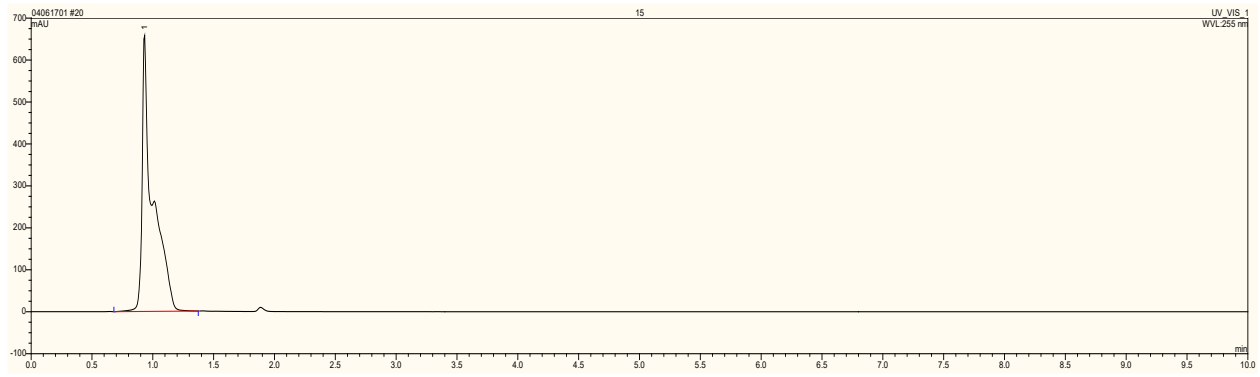

Fraction 16

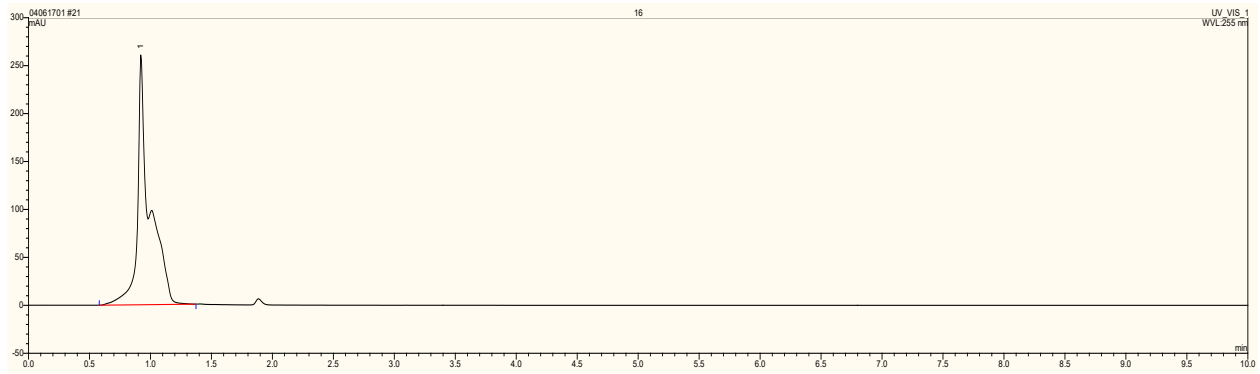


Fraction 17

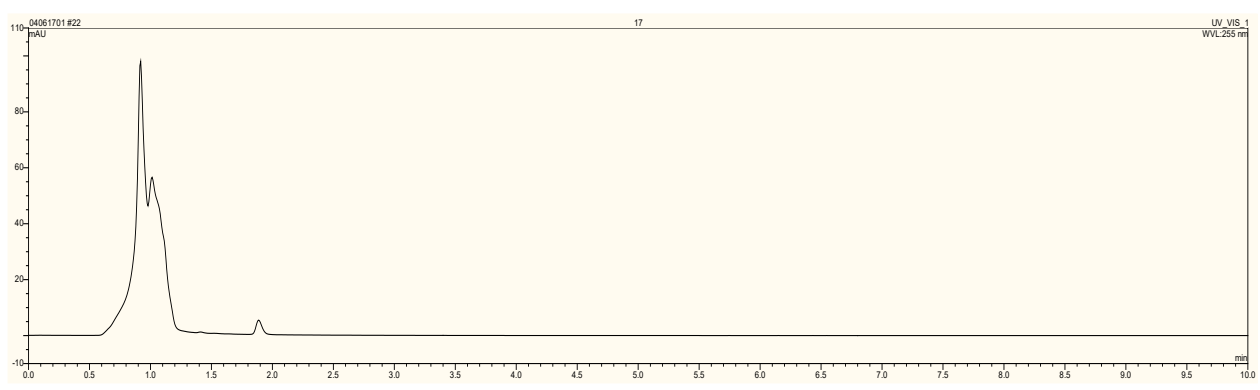

Fraction 18

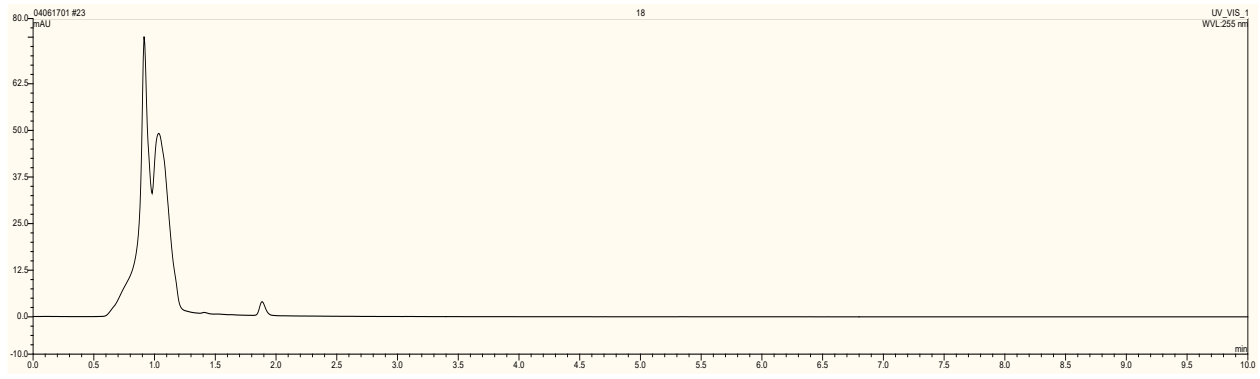

Fraction 19

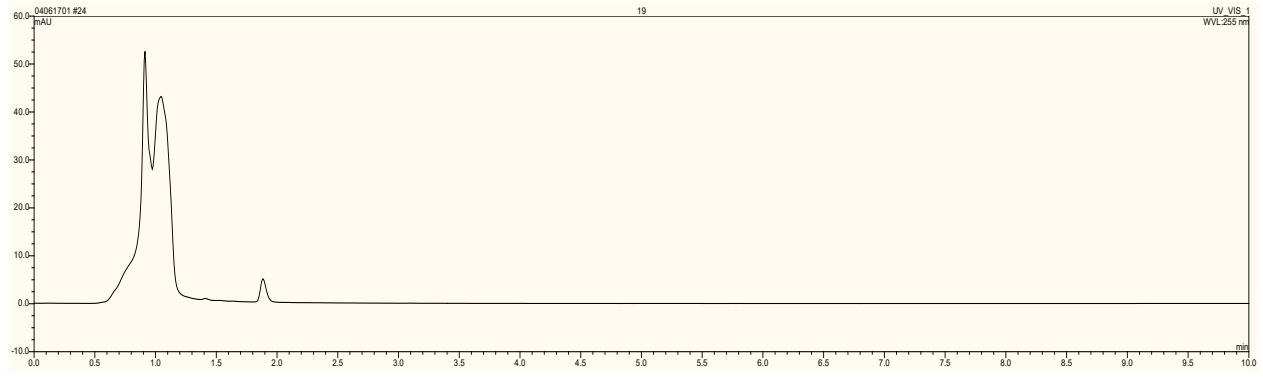

Fraction 20

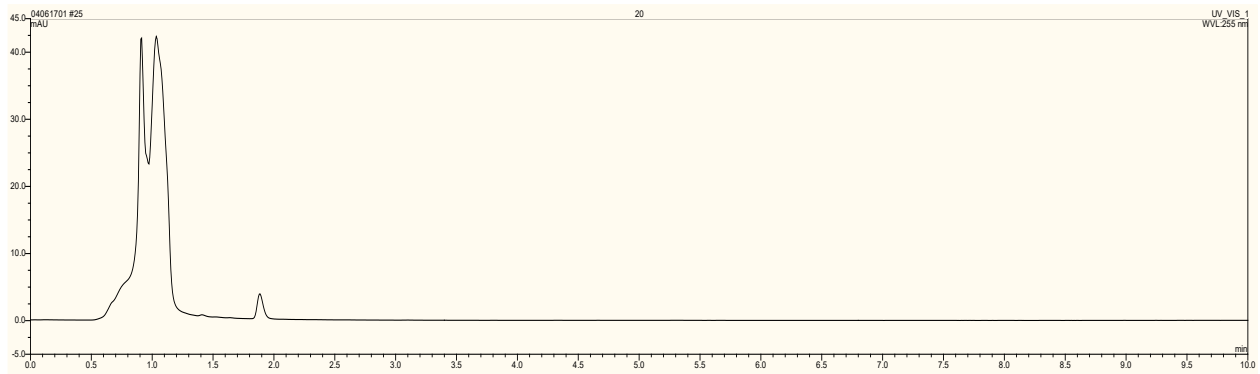


Fraction 21

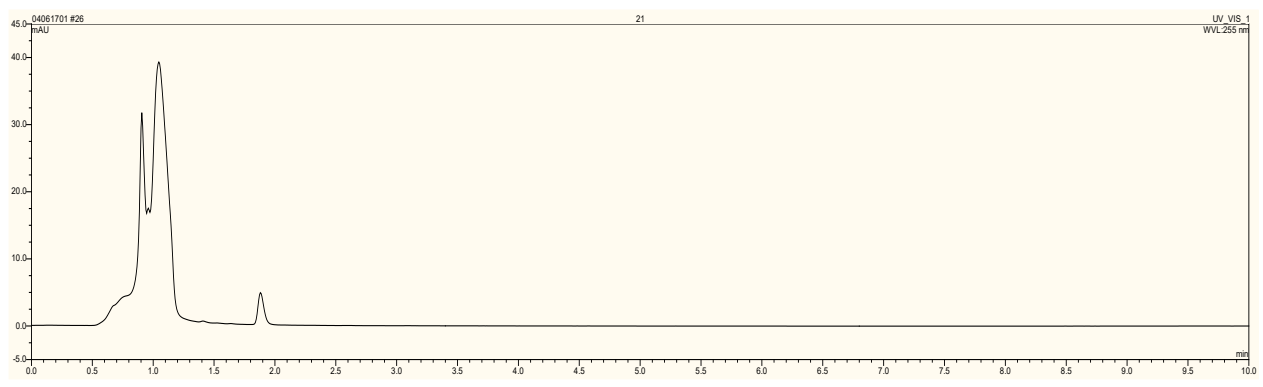

Fraction 22

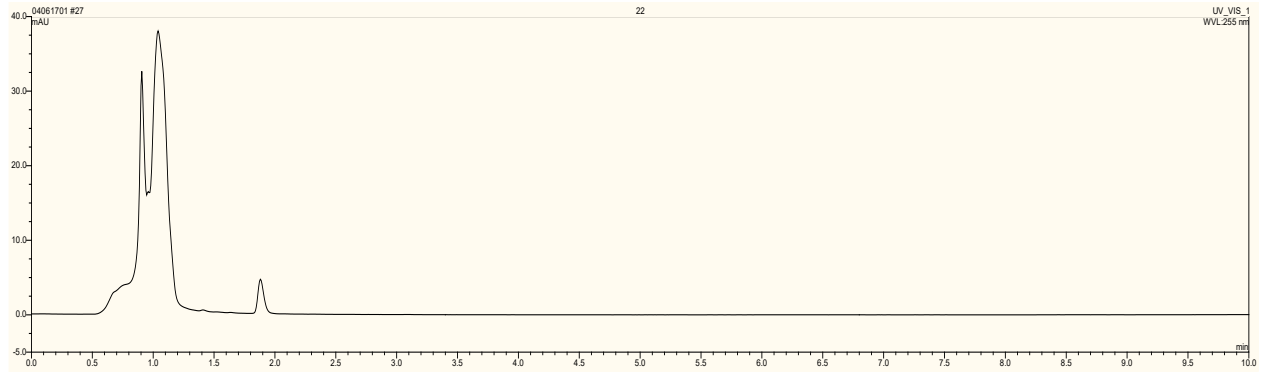

Fraction 23

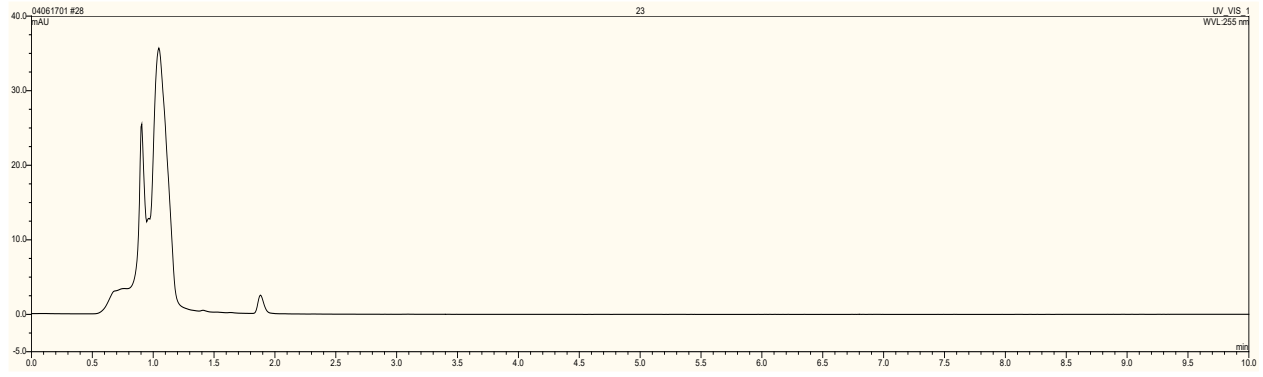

Fraction 24

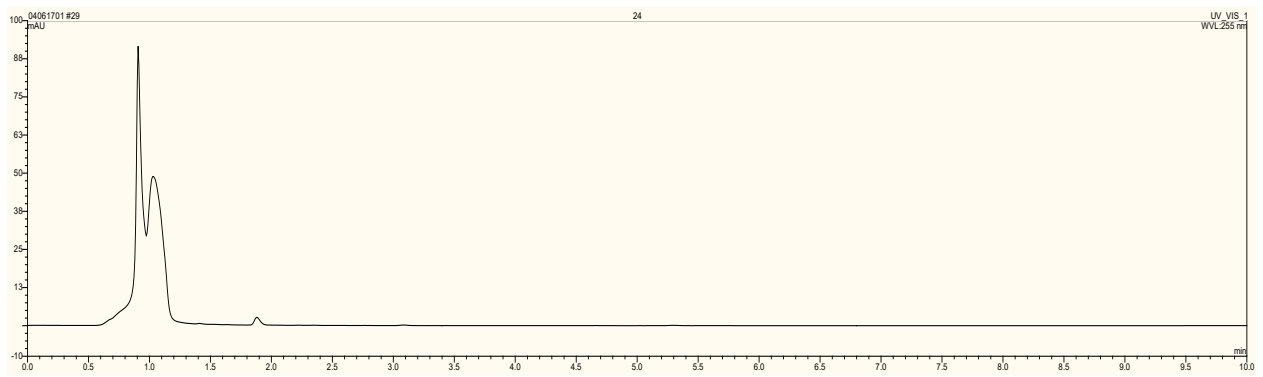


Fraction 25

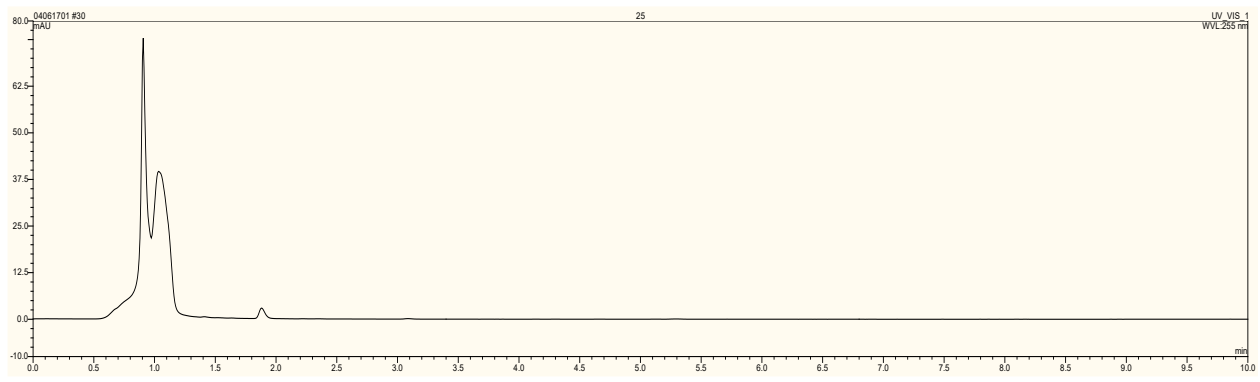

Figure 2.2: HPLC chromatogram of the 25 fractions collected via column chromatography. Each fraction was analyzed on a C18 column with a 50/50 methanol/acetonitrile mobile phase, flow rate $1 \mathrm{ml} / \mathrm{min}$, UV $255 \mathrm{~nm}$.

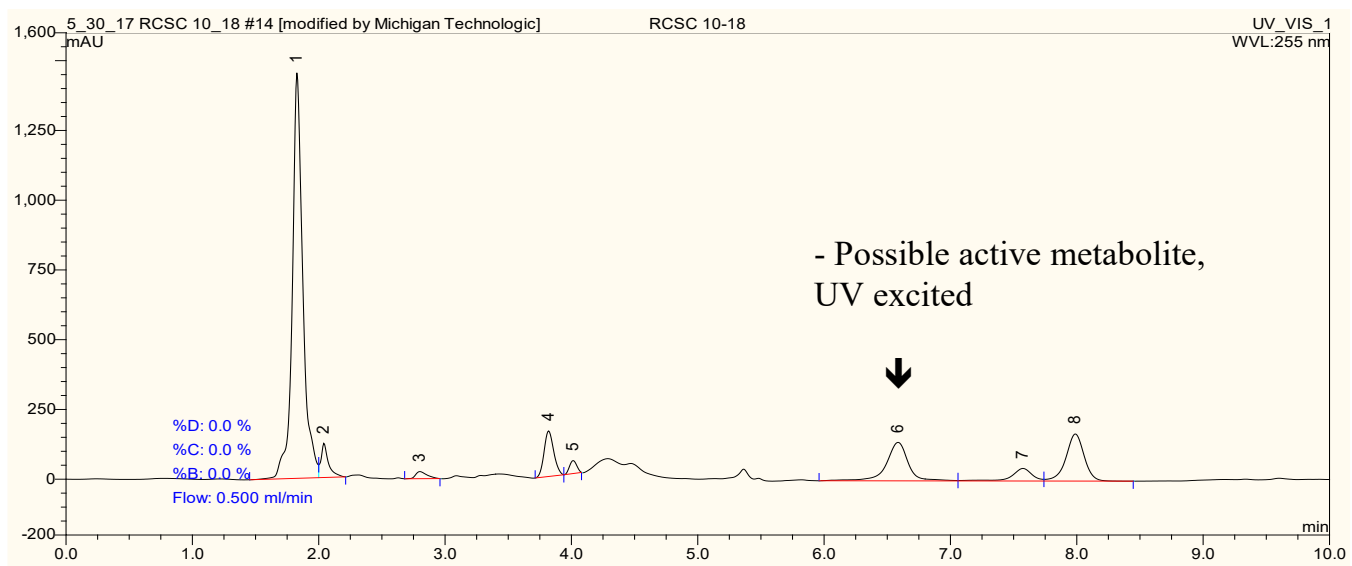

Figure 2.3: HPLC chromatogram of fractions 10-18. Fractions analyzed on a C18 column with a $100 \%$ HPLC grade water mobile phase, $.5 \mathrm{ml} / \mathrm{min}$ flow rate, $10 \mathrm{~min}$ total run time, UV $255 \mathrm{~nm}$. 


\subsubsection{RCSC and concentrates induced rapid cell death in human colon adenocarcinoma cell line SW620}

RCSC was tested for anticancer activity using propidium iodide staining with fluorescent microscopy and flow cytometry against the human colon adenocarcinoma cell line, SW620. Furthermore, a dose response curve was constructed using 1x, 3x, 6x and 33x concentrated RCSC. Treatment of SW620 with a 1:5 dilution of RCSC after 5 minutes resulted in minor increase in fluorescence, while after 10 minutes significant increase in fluorescence was observed (Figure 2.4).

A.

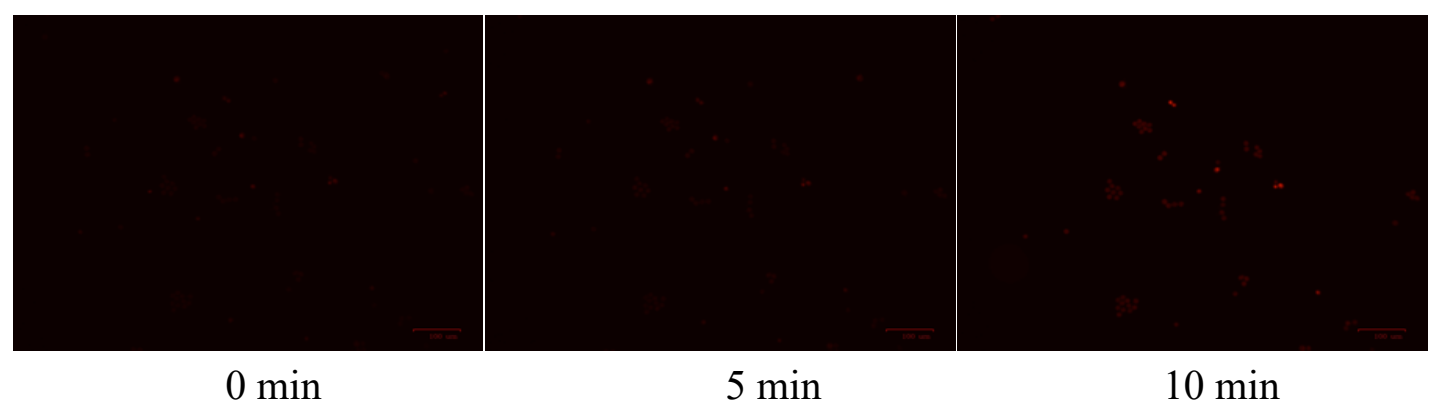

B.

Time course normal RSCS

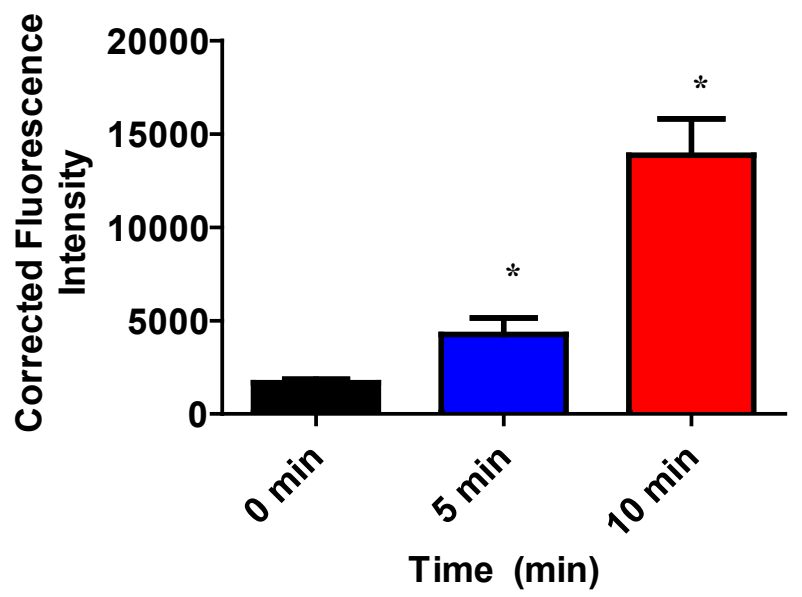


Figure 2.4: RCSC induces rapid cell death on the human colon adenocarcinoma cell line SW620 as analyzed by fluorescent microscopy. A. Real time imaging of SW620 assayed with propidium iodide cell viability assay following treatment with a 1:5 dilution of RCSC. Pictures acquired at 5 min intervals. Images taken using a Leica inverted fluorescent microscope at 200x, excitation/emission 533nm/617nm. B. Quantification of real time imaging of $\mathrm{RCSC}$ treatment with propidium iodide cell viability assay. *= indicates significance vs. non treated control. Results analyzed utilizing one way ANOVA with Tukeys test $(\mathrm{n}=5, \mathrm{p}<0.05)$.

SW620 analyzed by flow cytometer following a 1:5 dilution for 30 minutes of 1x, $3 x, 6 x$, and 33x RCSC resulted in a dose dependent death response based on propidium iodide staining (Figure 2.5). RCSC increased cell death to $7.7 \%$, 3x concentrated RCSC to $18.9 \%, 6 \mathrm{x}$ RCSC to $77.1 \%$, and $33 \mathrm{x}$ to $78 \%$ versus untreated control at $5 \%$ (Figure 4A). Additionally, the dose response curve calculated using the above concentrations resulted in an $\mathrm{EC}_{50}$ dilution factor being 3.64 (Figure 2.5). The $\mathrm{EC}_{50}$ value represents the dilution factor of the compound in which $50 \%$ of the maximal effect is observed, in this case $50 \%$ of the SW620 cells would die following treatment with RCSC at a 1:3.64 dilution of the $3 \mathrm{x} / 6 \mathrm{x}$ concentrated RCSC. Data was analyzed utilizing one-way ANOVA with Tukey's test $(\mathrm{p}<0.05)$ for both fluorescent microscopy and flow cytometry $(n=5)$. 
A.

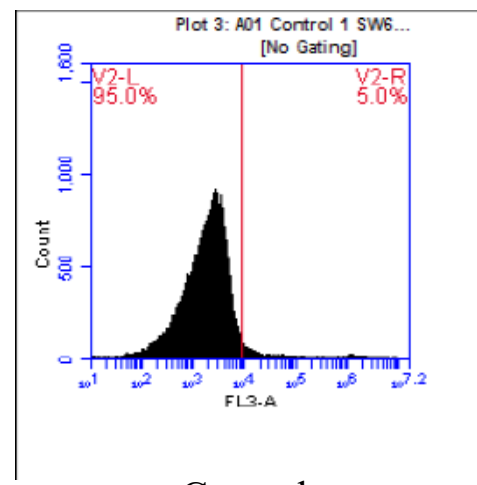

Control

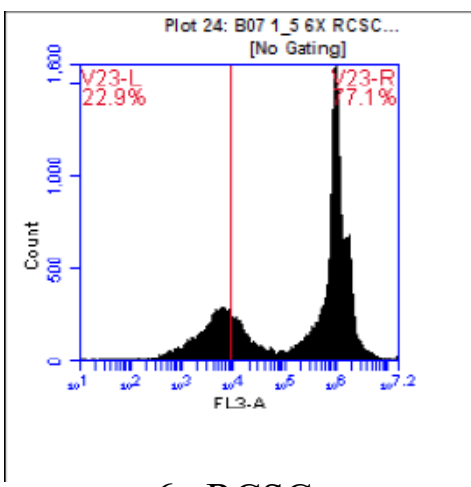

6x RCSC

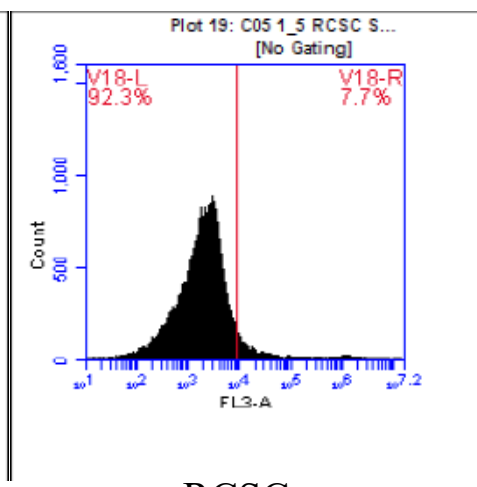

RCSC

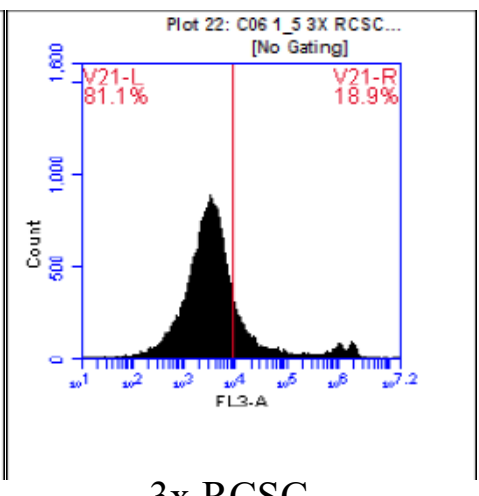

3x RCSC

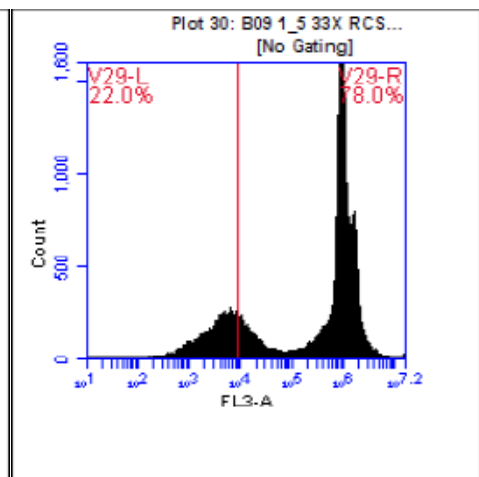

33x RCSC

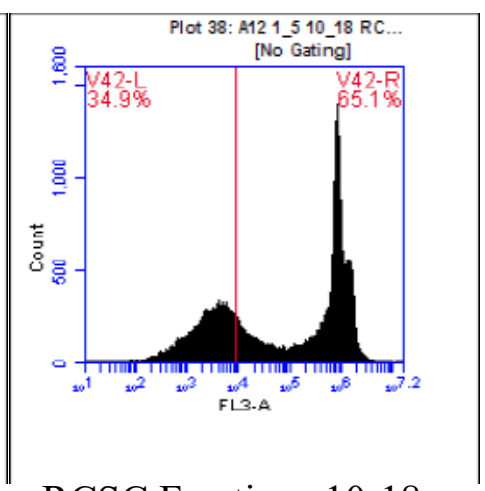

RCSC Fractions 10-18

B.

RCSC Dose Response log SW 620

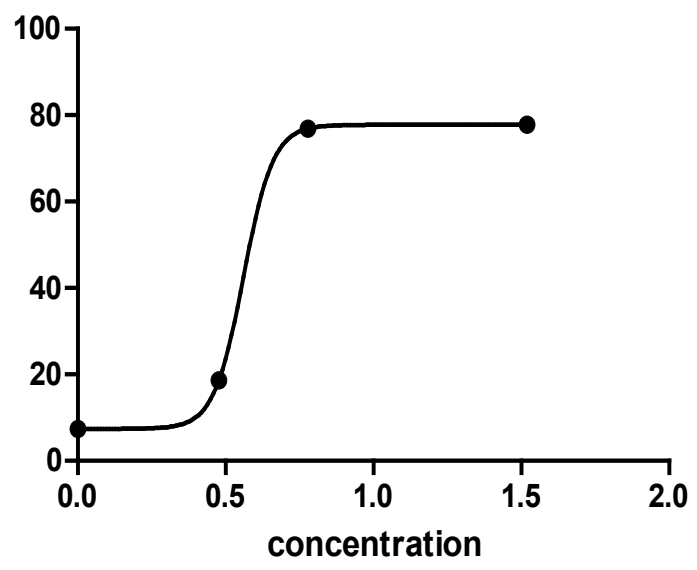

$\mathrm{EC}_{50}=3.64$ dilution factor 
C.

SW620 RCSC Dose

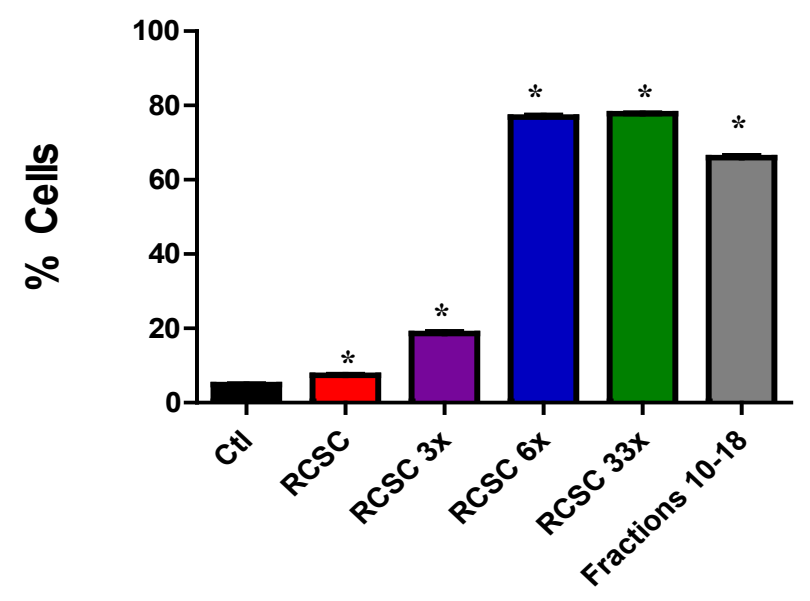

Figure 2.5: RCSC, concentrates, and fractions 10-18 rapidly induce cell death following 30-minute treatments of a 1:5 dilution. A. The colon adenocarcinoma cell line SW620 treated for 30 minutes with RCSC, concentrates, and fractions 10-18 at a 1:5 dilution utilizing propidium iodide cell viability assay. B. Graphical representation of the lowest effective dose, $\mathrm{EC}_{50}$ value, calculated using the varying concentrates. C. Bar graph showing dose response of RCSC concentrates on SW620 cell line. *= indicates significance vs. non treated control. Results analyzed utilizing one way ANOVA with Tukeys test (FL3 laser 670nm, p<0.05).

\subsubsection{RCSC and fractions 10-18 significantly increased cell death in human colon adenocarcinoma cell line SW620 comparable to whole RCSC}

Fractions 10-18 were analyzed by fluorescent microscopy and flow cytometry with propidium iodide staining for anti-cancer activity against the adenocarcinoma cell line, SW620. Similar HPLC peaks resulted in pooling of the selected fractions for testing, 
as they likely contained the same compounds. A 1:5 dilution was utilized for testing of the fractions, as this dilution factor resulted in the greatest inhibitory effect by RCSC on SW620. Following a 30-minute treatment with fractions 10-18, significant increases in fluorescence versus untreated control was observed when viewed under Leica fluorescent microscope indicating a reduction in the number of viable cells (Figure 2.6). Additionally, cells showed characteristics of apoptosis such as nuclear condensation and visual apoptotic bodies (Figure 2.6).

A.

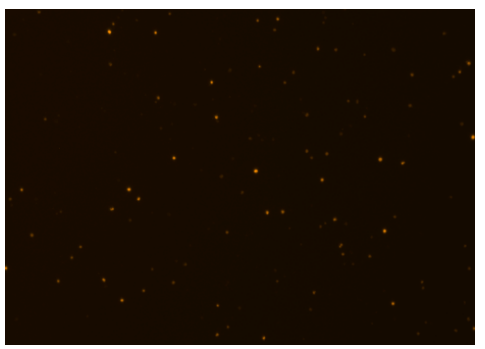

Control

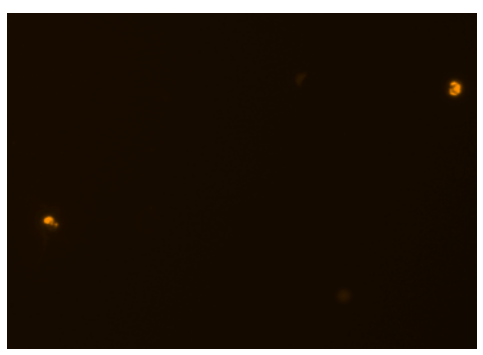

Control

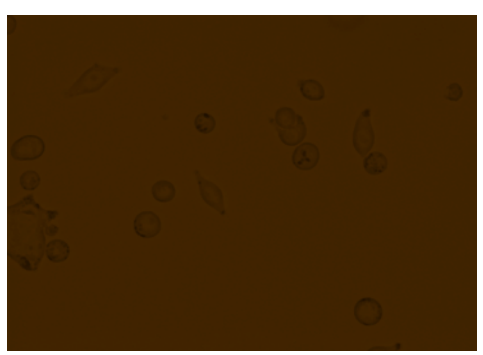

Control

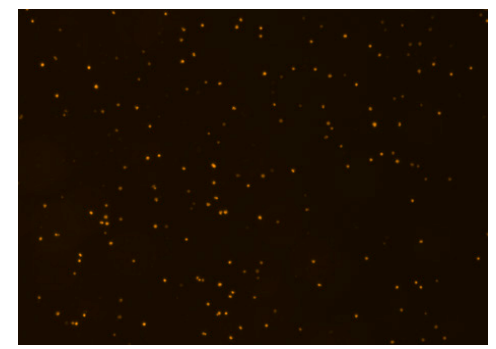

RCSC Fractions 10-18

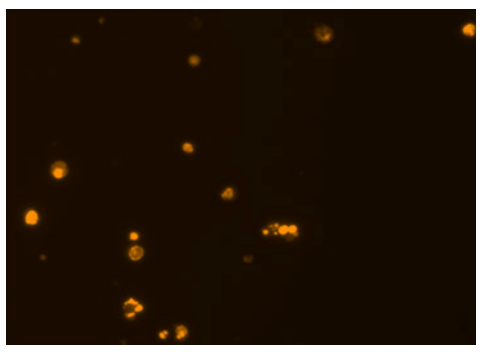

RCSC Fractions 10-18

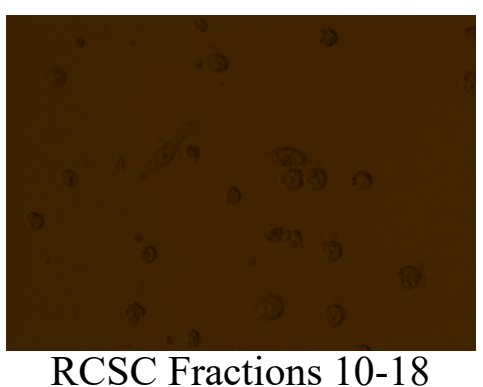




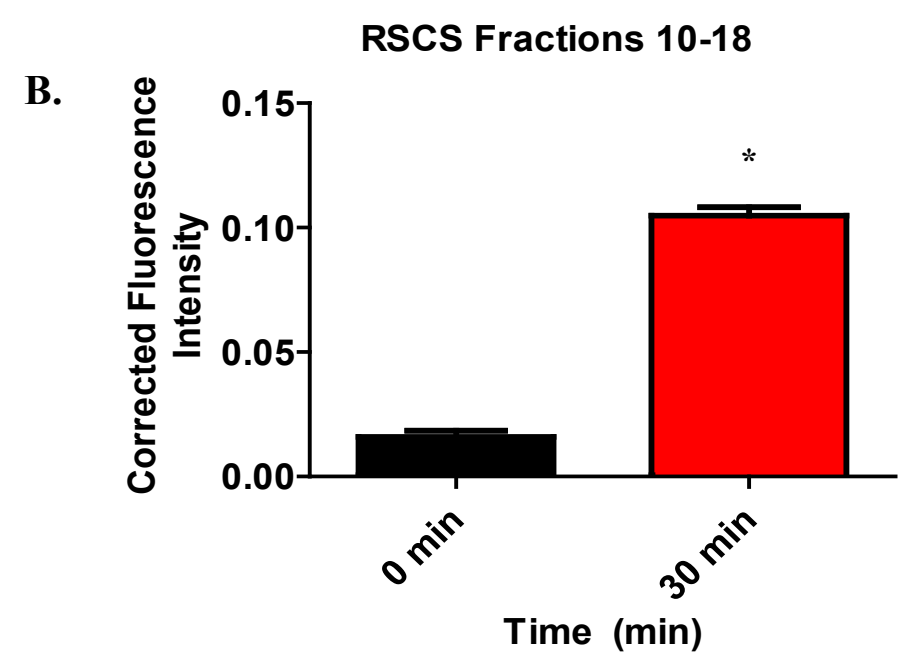

Figure 2.6: RCSC fractions 10-18 induced rapid cell death in the human colon adenocarcinoma cell line SW620 as analyzed by fluorescent microscopy. A. Real time imaging of SW620 assayed with propidium iodide cell viability assay following treatment with a 1:5 dilution of RCSC fractions 10-18. Pictures acquired at 30 min intervals. Images taken using a Leica inverted fluorescent microscope at 200x, excitation/emission $533 \mathrm{~nm} / 617 \mathrm{~nm}$. B. Quantification of real time imaging of RCSC fractions $10-18$ treatment with propidium iodide cell viability assay. $*=$ indicates significance vs. non treated control. Results analyzed utilizing one way ANOVA with Tukeys test $(\mathrm{n}=5, \mathrm{p}<0.05)$.

Furthermore, when flow cytometry was used to analyze propidium iodide stained cells, fractions 10-18 revealed significant increase in cell death versus untreated control, recording $65.1 \%$ and $5 \%$ dead cells, respectively (Figure 2.5). Data was analyzed utilizing one-way ANOVA with Tukey's test $(n=5, p<0.05)$ for both fluorescent microscopy and flow cytometry. 


\subsubsection{RCSC and concentrates mildly increased cell death in normal human colon cell line CCD 841 CoN}

RCSC and concentrates were tested for effects on cell death using the normal colon cell line CCD $841 \mathrm{CoN}$ with propidium iodide staining and flow cytometry. CCD $841 \mathrm{CoN}$ was treated for 48 hours with $1 \mathrm{x}, 3 \mathrm{x}, 6 \mathrm{x}$, and $33 \mathrm{x}$ concentrated RCSC for 48 hours. Cell death increased slightly in the $1 \mathrm{x}$ RCSC group to $9.6 \%, 11.8 \%$ in the $3 \mathrm{x}$ treated, $6 \mathrm{x}$ to $21.4 \%$, and $33 \mathrm{x}$ to $22.1 \%$ versus $4.4 \%$ in the untreated control (Figure 2.7 ). Furthermore, a dose response curve was generated using the above concentrates and resulted in $\mathrm{EC}_{50}$ dilution factor of 4.38 (Figure 2.7). Data was analyzed utilizing one-way ANOVA with Tukey's test $(\mathrm{p}<0.05)$.

A.

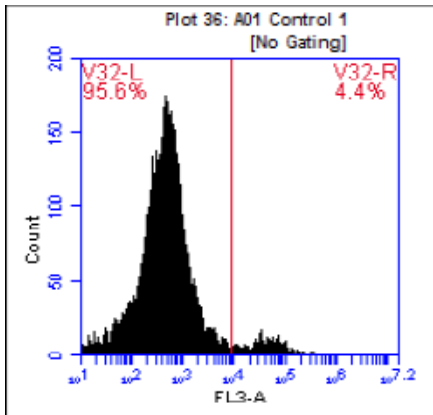

Control

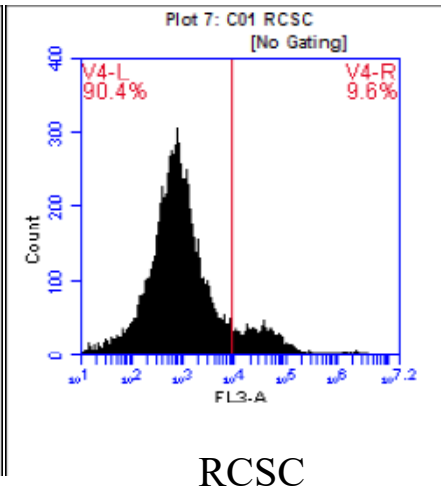

RCSC

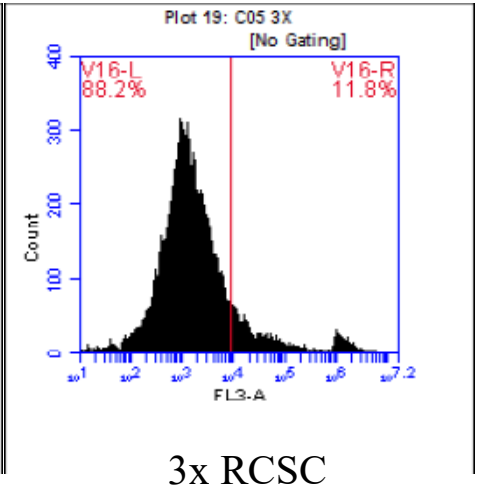

3x RCSC 


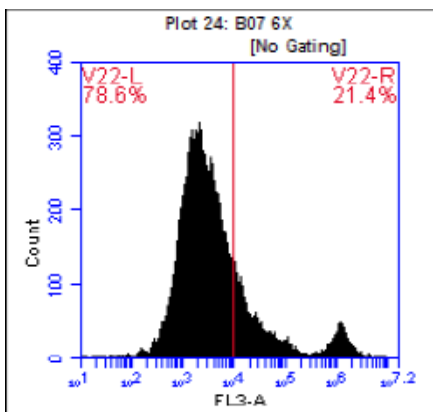

6x RCSC

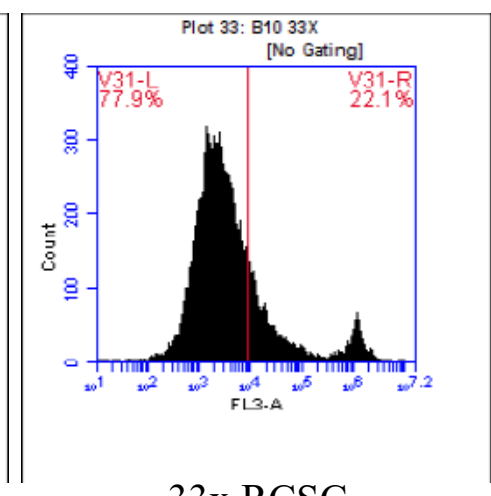

33x RCSC

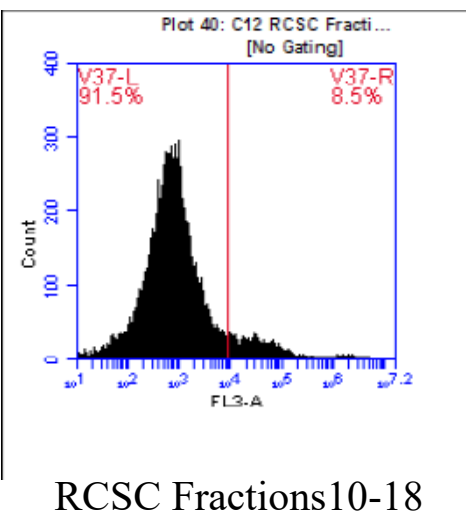

RCSC Fractions10-18

B.

RCSC Dose Response log

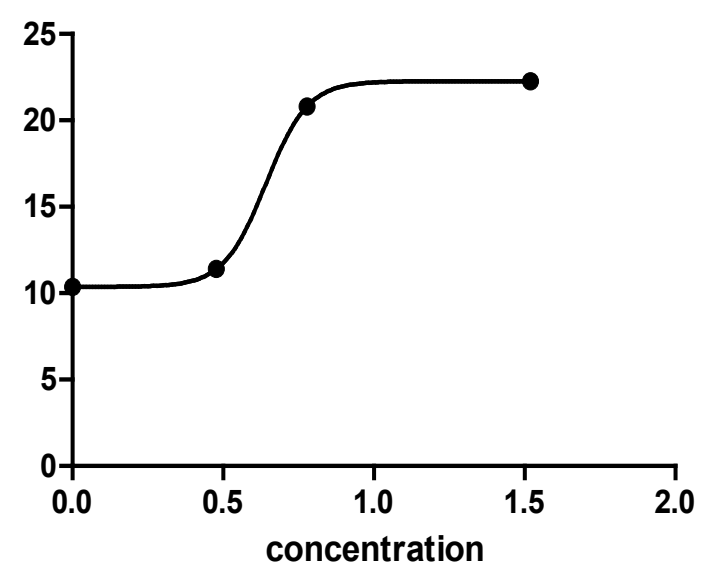

$\mathrm{EC}_{50}=4.38$ dilution factor

C. CCD RCSC Dose

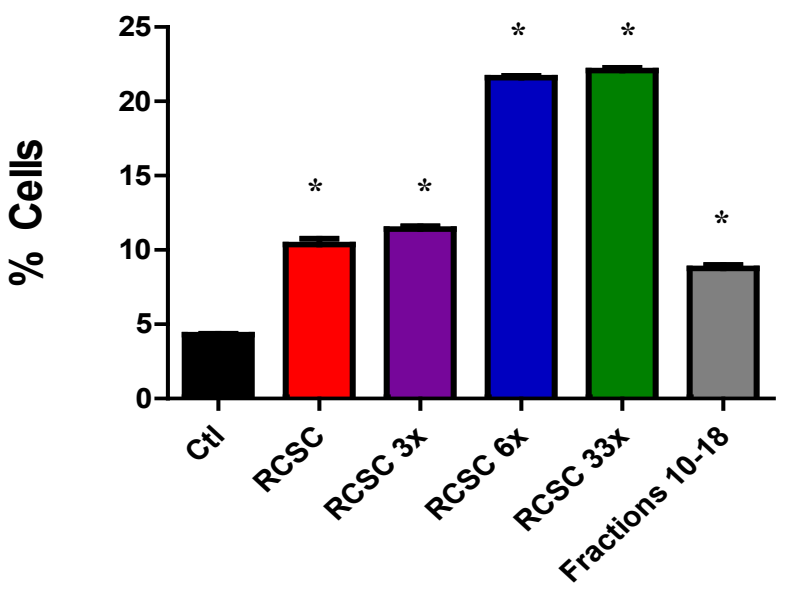


Figure 2.7: RCSC, concentrates, and fractions 10-18 had minimal toxicity in the normal colon cell line CCD 841 CoN. A. The normal colon cell line CCD 841 CoN was treated for 30 minutes with RCSC, concentrates, and fractions 10-18 at a 1:5 dilution utilizing propidium iodide cell viability assay. B. Graphical representation of the lowest effective dose, $\mathrm{EC}_{50}$ value, calculated using the varying concentrates. C. Bar graph showing dose response of RCSC, concentrates, and fractions 10-18 on CCD $841 \mathrm{CoN}$ cell line. $*=$ indicates significance vs. non treated control. Results analyzed utilizing one way ANOVA with Tukeys test (FL3 laser 670nm, p<0.05).

\subsubsection{RCSC fractions $10-18$ resulted in mild increases in cell death, but showed less toxicity than whole RCSC on the normal human colon cell line CCD 841 CoN}

Fractions 10-18 were tested for cell death on normal human colon cell line CCD $841 \mathrm{CoN}$ using propidium iodide and flow cytometry. Mild increase in fractions $10-18$ versus untreated control fluorescence $(8.5 \%$ versus $4.4 \%)$ due to propidium iodide was observed (Figure 2.7). Additionally, fractions 10-18 were collected using 33x concentrated RCSC that showed $22.1 \%$ fluorescence corresponding to dead cells. These results indicate that the purification of fractions $10-18$ reduced the toxicity in $33 \mathrm{x}$ concentrated RCSC. Data was analyzed utilizing one-way ANOVA with Tukey's test $(\mathrm{p}<0.05)$ for flow cytometry $(\mathrm{n}=5)$. 


\subsubsection{RCSC fractions 10-18 increased nuclear and cytoplasmic ROS in the normal human colon cell line CCD 841 CoN}

RCSC fractions 10-18 were tested for ROS scavenging capabilities following 24hour treatment with the pro-inflammatory cytokines, TNF- $\alpha$, IL- $1 \beta$, IFN- $\gamma$, and LPS. Following a 48-hour treatment period, nuclear ROS increased to $8.8 \%$ compared to $4.8 \%$ in untreated control, and 5.8\% in inflammation treated control, as analyzed by flow cytometry under FL1 (Figure 2.8).

A.

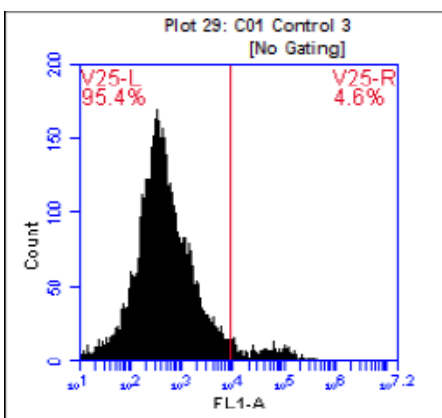

Control
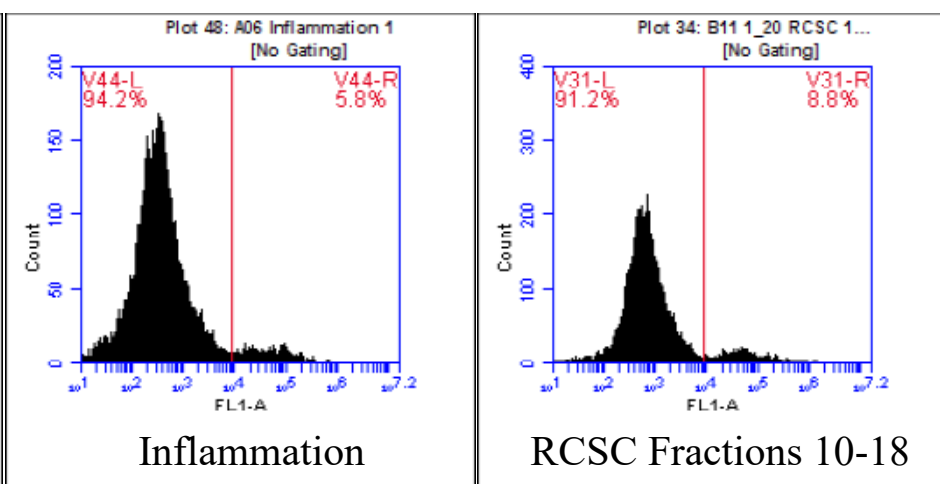

B.

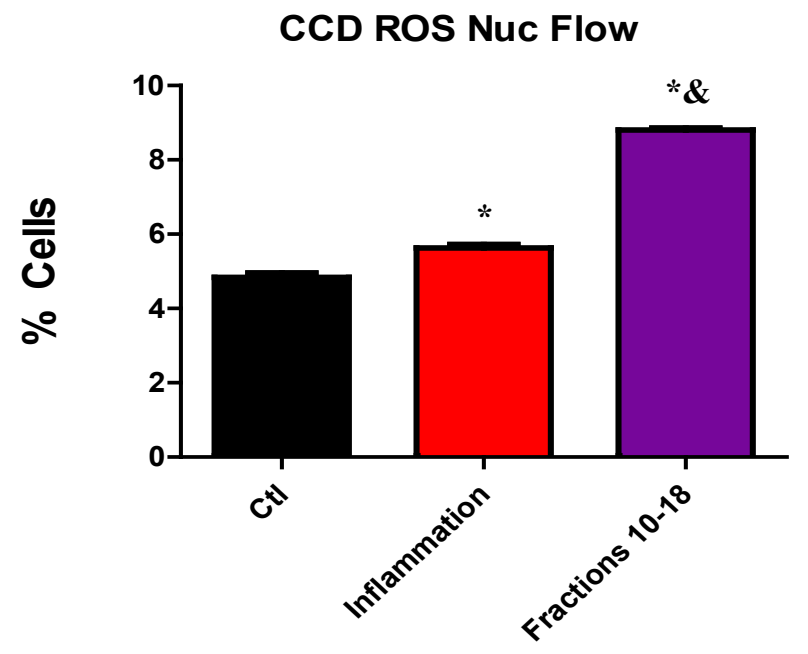


Figure 2.8: RCSC fractions 10-18 increased nuclear ROS in CCD 841 CoN similarly to whole RCSC as analyzed by flow cytometer. A. CCD $841 \mathrm{CoN}$ cells were treated with the pro inflammatory cytokine cocktail for 24 hours and RCSC fractions $10-18$ for an additional 48 hours before analysis using CellROX® Green nuclear stain under fluorescent laser one $(530 \mathrm{~nm}) . *=$ indicates significance vs. non treated control, $\&=$ indicates significance vs. inflammatory control. Results analyzed utilizing one-way ANOVA with Tukeys test, $\mathrm{p}<0.05$. Analysis was done on $\mathrm{BD}$ Accuri ${ }^{\mathrm{TM}} \mathrm{C} 6$ flow cytometer.

Cytoplasmic ROS also increased following treatment with fractions 10-18 to $7.4 \%$ versus $3.7 \%$ in untreated control group, $4.8 \%$ in inflammation treated group, as analyzed by flow cytometer under FL3 (Figure 2.9). Data was analyzed utilizing one-way ANOVA with Tukey's test $(\mathrm{p}<0.05)$ for flow cytometry $(\mathrm{n}=5)$.

A.
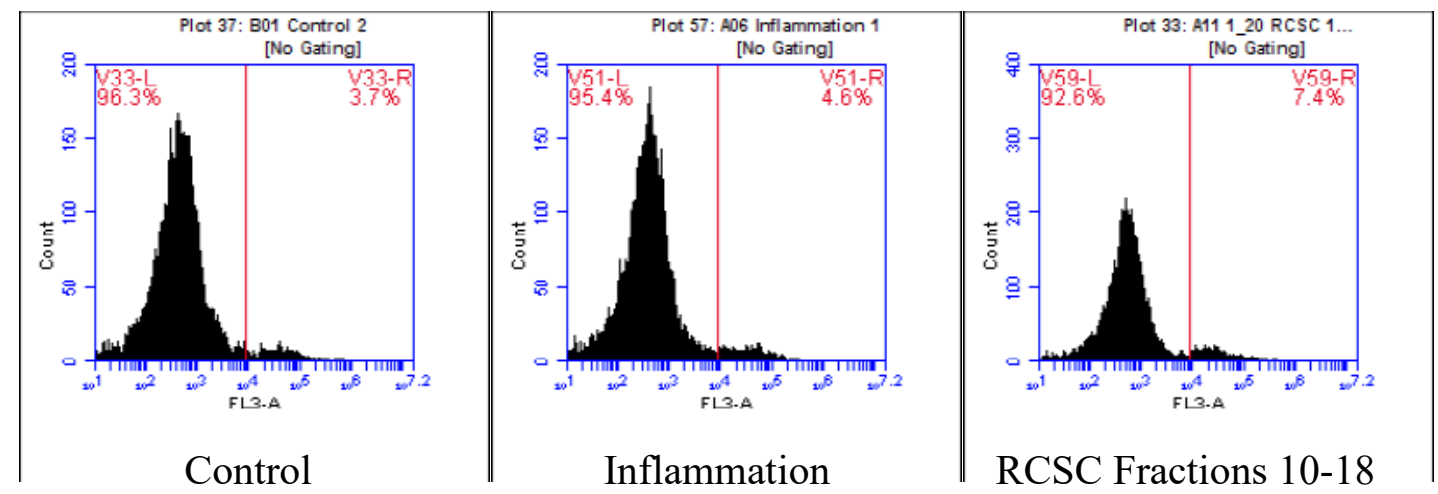
B.

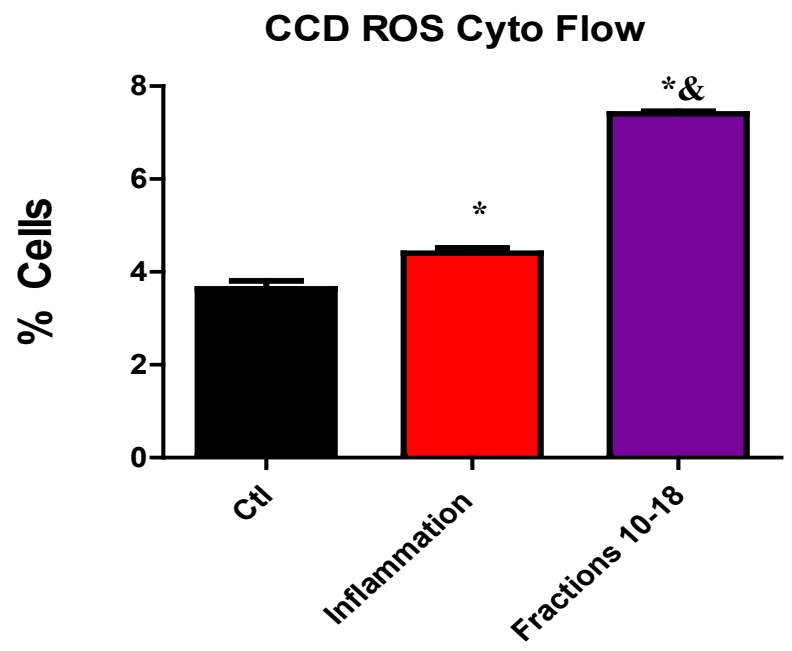

Figure 2.9: RCSC fractions 10-18 increased cytoplasmic ROS in CCD 841 CoN similarly to whole RCSC as analyzed by flow cytometer. A. CCD $841 \mathrm{CoN}$ cells were treated with the pro inflammatory cytokine cocktail for 24 hours and RCSC fractions 1018 for an additional 48 hours before analysis using CellROX ${ }^{\circledR}$ orange cytoplasmic stain under fluorescent laser three $(670 \mathrm{~nm}) . *=$ indicates significance vs. non treated control, $\&=$ indicates significance vs. inflammatory control. Results analyzed utilizing one-way ANOVA with Tukeys test, $\mathrm{p}<0.05$. Analysis was done on BD Accuri ${ }^{\mathrm{TM}} \mathrm{C6}$ flow cytometer.

\subsection{Discussion}

Our current aim was to identify the possible bioactive compounds in RCSC responsible for the anti-cancer, and anti-inflammatory effects as described in previous literature published and unpublished ${ }^{12,13}$. Although we were unable to identify a specific 
compound responsible for such activity, we were able to identify 8 peaks which most likely correspond to specific compounds, as described above, with activity similar to previous reports.

Several different extraction solvents were used and then spotted for possible compound identification, methanol and/or methanol/chloroform however seemed to be superior in separating the compounds isolated from the aqueous solution. When running the samples with HPLC on a C18 column, an aqueous mobile phase seemed to increase separation on the column compared to using methanol alone as the mobile phase. Additionally, we knew the compound shows absorbance in UV range, as the longer the incubation period for generating RCSC, the greater the absorbency. This gave us another clue as to how to identify the possible fraction based on UV absorption spectrum. Furthermore, absorbance in UV spectrum provides information about the chemical structure of the compound or compounds. RCSC fractions 10-18 showed absorbance in UV range and when further analyzed by HPLC, peak 6 maintained the absorbance when exposed to UV light. In most cases, this is indicative of conjugation or functional groups within the compounds. Furthermore, when methanol was rotary-evaporated, a sap like substance was produced which exhibited poor solubility in water and PBS, suggestive of a more hydrophobic compound.

In order to understand if RCSC fractions 10-18 were responsible for anti-cancer and anti-inflammatory activity, we utilized the colon adenocarcinoma cell line, SW620 and the normal colon cell line, CCD $841 \mathrm{CoN}$ with various assays as described above. Fractions 10-18 were collected using the $33 \mathrm{X}$ concentrated RCSC sample, therefore both 
should contain roughly the same concentrations for testing purposes. When it came to testing RCSC and/or the fractions on SW620, both reported high anti-cancerous activity (>65\% death). Furthermore, when RCSC and fractions $10-18$ were tested on the normal colon cell line CCD $841 \mathrm{CoN}$, fractions $10-18$ were less toxic on the normal cell line versus all other RCSC samples (1X, 3X, 6X, and 33X). This is possibly due to the concentration of the active compound(s) (unknown), or from the removal of any toxic compounds in the aqueous RCSC solution. In either case, RCSC fractions 10-18 continued to exhibit selectivity towards cancerous cell lines, with minimal toxicity towards normal cells.

Previous unpublished literature suggests RCSC to have anti-inflammatory and immune-modulating properties. To identify if these properties were maintained in RCSC fractions 10-18, CellROX ${ }^{\mathrm{TM}}$ green and orange dyes were tested for free radical scavenging capabilities. RCSC alone increased both nuclear and cytoplasmic generated ROS, while increasing cell survivability following treatment with a pro-inflammatory cytokine cocktail. We concluded fractions 10-18 to exhibit similar results, however the response was reduced.

ROS has been shown to participate in a multitude of signaling pathways responsible for both the induction and removal of the inflammatory response ${ }^{57}$. To this day, there is no clear-cut answer as to whether or not it plays a beneficial or detrimental role in biological systems. We do however know cancer cells generate and utilize high amounts of free radicals to initiate proliferation as well as increase antioxidant defense mechanisms ${ }^{57}$. This crucial need to maintain homeostasis can result in the explosion or 
elimination of tumorigenic cells. In regards to cancer therapy, a review on treatments with antioxidants $\beta$-carotene, vitamin $A$, and vitamin $C$ resulted in no significant benefit and in some cases resulted in deleterious effects ${ }^{57,58}$. Moreover, there is accumulating evidence for the use of small molecules, which are able to selectively target and disable the antioxidant pathways utilized in cancer cells ${ }^{59-61}$.

In inflammatory systems, ROS homeostasis is crucial for generating the appropriate immune response. ROS seems to be intimately linked with both T- and Bcell function. Administration of antioxidants seems to diminish both T- and B- cell receptor signaling, which is crucial for mounting the proper immune response. As in tumorigenic tissue, generation and elimination of ROS determines if the response is beneficial or detrimental. For example, in mice lacking uncoupling protein 2 (UCP2), elevated ROS levels increased their immunity to bacterial infections, thus indicating a beneficial role of moderate increases in ROS levels. Additionally, mice heterozygous for mitochondrial hydroxylase necessary for ubiquinone synthesis (Mclk1) have elevated mitochondrial ROS levels along with elevated innate and adaptive immune responses that aid in pathogen defenses without harming surrounding tissue ${ }^{62}$. On the contrary, antioxidants have shown to be beneficial in NRF2-deficient mice, because of their inability to regulate ROS generation and elimination ${ }^{63,64}$. Lastly, ROS is an inevitable byproduct of aerobic metabolism, while several decades of research have been focused on the notion of ROS being responsible for several deleterious biological effects; this seems not to be the case ${ }^{65-67}$. Instead, recent evidence is strongly suggestive of ROS being required for proper maintenance of immune function and positively promotes healthy 
tissue generation and repair ${ }^{68,69}$. In conclusion, the responses observed in our study of increased ROS levels as a result of treatment with RCSC fractions 10-18 containing the bioactive compound or compounds responsible suggest anti-cancerous and antiinflammatory activity.

\subsection{Conclusion and future directions}

RCSC fractions 10-18 exhibit similar responses as whole RCSC on both colon adenocarcinoma cell line SW620 and the normal colon cell line CCD 841 CoN indicating the possibility of one or more of the compounds corresponding to eight peaks being responsible for the observed effects. The next step would be to isolate the individual compounds and test for efficacy. From there, structural determinations would need to be performed to gain a greater understanding of possible biochemical and molecular targets. Finally, isolation of a single compound could provide a new anticancer and antiinflammatory compound with future clinical applications.

\subsection{Acknowledgements}

This work was supported by the National Institute of Food and Agriculture, U.S. Department of Agriculture, under award number 2014-67018-21767. 
Chapter 3: Anti-inflammatory Effects of Northern Highbush Blueberry Extract on Two Inflamed Colon Epithelial Cell Lines 


\subsection{Abstract}

Blueberries have been described for decades as a "super food". Several epidemiological studies have indicated blueberry polyphenols to exhibit potent antiinflammatory, anti-tumorigenic, and anti-oxidant capabilities in-vivo and in-vitro. While found abundantly within the human diet, the bioavailability of these compounds remains localized to the gastrointestinal tract (GI). Several studies have indicated that blueberry anthocyanins have the ability to efficiently reach the GI tract and exhibit a broad range of biochemical effects. Furthermore, in the context of inflammatory bowel disease (IBD), blueberry anthocyanins remain a promising complement to current IBD treatments. Here, we investigated the antiinflammatory and antioxidant capabilities of Highbush blueberries in-vitro on two normal colon epithelial cell lines, NCM 356 and CCD 841 CoN, following stimulation with a pro-inflammatory cytokine cocktail. Antioxidant and antiinflammatory properties were examined via fluorescent microscopy and flow cytometry utilizing various fluorescent probes. Treatment with phenolic rich blueberry extract revealed drastic decrease in nuclear and cytoplasmic generated reactive oxygen species (ROS) compared to controls. Additionally, the phenolic rich blueberry extract increased cell viability following treatment with the proinflammatory cytokine cocktail. Here, in conjunction with current literature, we describe a phenolic rich blueberry extract to exhibit potent anti-inflammatory and anti-oxidant capabilities. 


\subsection{Introduction}

\subsubsection{Inflammation and Inflammatory Bowel Disease (IBD)}

Inflammation has been described for as long as medicine itself has been around. In ancient times, it was simply described as red, hot, swollen skin ${ }^{70}$. It wasn't until the late 1800s when Julius Friedrich Cohnheim first revealed the classical understanding of inflammation; vasculature changes, infiltration of white blood cells, and systemic changes $^{71}$. Furthermore, fast forward 100 years later to 1977 and this still holds true, albeit a more specific and modified understanding ${ }^{70}$. Today, our understanding has vastly expanded. Inflammation, in general, is a response to any injury with a sole purpose to heal the affected tissue. This begins with a multitude of events, including activation and migration of neutrophils, monocytes, and eosinophils ${ }^{71,72}$. From there, a more specific response is initiated via the cross talk with cytokines and chemokines. These work as chemoattractant molecules to signal specific leukocyte populations, dependent on injury $^{72}$. Additionally, this response can either be acute or chronic, dependent on the duration of the mediating factors. Wound healing for example is usually an acute response, while viral infections can lead to a more chronic response and possible neoplastic growth ${ }^{72}$.

Inflammatory bowel disease (IBD) includes a multitude of inflammatory conditions affecting the gastrointestinal tract, notably of which Crohn's disease (CD) and ulcerative colitis (UC) are the most common $^{73}$. These conditions affect roughly 1.4 million people in the US and Canada, with increasing incidents worldwide ${ }^{18,73}$. Ulcerative colitis is defined by specific pathological features, which include diffused inflamed 
mucosa, severe inflammation, and production of a plethora of inflammatory chemical mediators. On the other hand, the pathology of CD is characterized by the presence of macrophage aggregates, which produce non-necrotizing granulomas ${ }^{18}$. In either case, the general attribute of IBD is disrupted inflamed intestinal epithelium ${ }^{74}$.

The current treatment for IBD has been focused around maintenance of several pro-inflammatory cytokines, specifically $\mathrm{TNF} \alpha$ and IFN $\gamma^{20}$. Several studies in mouse models have implicated these cytokines, as well as the interleukin class of molecules in the pathogenesis of $\mathrm{IBD}^{20,75}$. These compounds are able to strongly regulate the immune response as well as drive intestinal and systemic inflammation ${ }^{20}$. For example, TNF $\alpha$ is able to regulate angiogenesis, Paneth cell death, macrophages, effector T cells, as well as the production of matrix metalloproteinases, all through binding to TNFR1 and TNFR2 receptors ${ }^{20,76-79}$. These actions, in turn, result in disruption of the epithelial barrier as well as apoptosis ${ }^{77}$. Additionally, IFN $\gamma$ has been shown to strongly increase vascular permeability and regulate cellular angiostasis in Dextran Sodium Sulfate (DSS) model of colitis in mice ${ }^{80}$.

Furthermore, while a plethora of literature suggests these cytokines and others as good targets for treatments, the reality is not as straightforward. Currently, the anti-TNF therapy drug, infliximab, has had mixed results, but it continues to be the first line of defense in treatment of $\mathrm{IBD}^{20}$. On the other hand, the anti-IFN $\gamma$ drug, fontolizumab, was unsuccessful in patients with $\mathrm{CD}^{20,81,82}$. This, along with the strong adverse side effects of similar anti-cytokine therapies, suggests a need for alternative and/or cooperative 
therapies $^{83}$. Dietary interventions are one of those alternatives, which have been shown to reduce and even eliminate inflammation through several different means.

\subsubsection{Anti-inflammatory effects of northern highbush blueberry extract polyphenols}

For decades, blueberries have been touted for their beneficial health claims, from anti-inflammatory and anti-tumorigenic, to providing protection against oxidative stress and chronic conditions ${ }^{84}$. These effects are known to come from a complex class of compounds, termed secondary metabolites ${ }^{29}$. Secondary metabolites are divided primarily into three groups, which include terpenoids, alkaloids, and phenylpropanoids and other

phenolic compounds ${ }^{1,5}$. Blueberries are an abundant source of the phenolic class of bioactive compounds. Over 10 compounds have been identified, with as many as 15 , with the content varying dependent on genetic and environmental factors ${ }^{85,86}$. For instance, highbush blueberries (Vaccinium corymbosum, L.) contain 15 different bioactive compounds, mainly delphinidin, cyanidin, petunidin, peonidin, and malvidin, with variation existing in number of hydroxyl groups, amount of methylation, as well as type and location of sugar molecules ${ }^{85,86}$. Wild blueberries (Vaccinium angustifolium), additionally, contain 15 different compounds, with differences being the identification of chlorogenic acid and an unknown compound ${ }^{84}$.

Our current aim is to investigate the anti-inflammatory properties of highbush blueberries on two inflamed colon epithelial cell lines: NCM 356 and CCD 841 CoN. Furthermore, we aim to mimic in vivo conditions of IBD by in vitro treatment with pro- 
inflammatory cytokines: interlukin 1 beta (IL-1 $\beta)$, tumor necrosis factor alpha (TNF- $\alpha$ ), interferon gamma (IFN-Y), and lipopolysaccharide (LPS).

\subsection{Materials and methods}

\subsubsection{Human colon cell lines}

Two human colon cell lines were used, NCM 356 and CCD 841 CoN. NCM 356 was licensed and obtained from INCELL (San Antonio, TX) for three years. NCM 356 was cultured and maintained in INCELL's M300A, 10\% fetal bovine serum (FBS) at $37^{\circ} \mathrm{C}, 5 \% \mathrm{CO}_{2}$ as per manufacturer recommendations. $\mathrm{CCD} 841 \mathrm{CoN}$ (ATCC® $\mathrm{CRL}-$ $1790^{\mathrm{TM}}$ ) was purchased from ATCC (Manassas, VA) and cultured in Eagle's minimum essential medium (EMEM, ATCC® $30-2003$ ) with $10 \% \mathrm{FBS}$ at $37^{\circ} \mathrm{C}, 5 \% \mathrm{CO}_{2}$ as per manufacturer recommendations. Penicillin-streptomycin-amphotericin B solution was purchased from ATCC (ATCC® PCS-999-002).

\subsubsection{Cell counting, freezing, and seeding protocol}

Cells were counted using a hemocytometer following the general trypan blue cell exclusion assay. CCD $841 \mathrm{CoN}$ was grown until $70-90 \%$ confluent and collected using trypsin EDTA. Cells were centrifuged and then re-suspended in the appropriate culture medium. Cells were counted utilizing the following formula: Number of cells $/ \mathrm{mL}=(\mathrm{X})$ $*(20) *(1.2) * 10^{4}$, where $\mathrm{X}=$ average number of cells in four squares, 20 = first dilution factor, and $1.2=$ second dilution factor. Seeding was done in 6, 12, 24, or 96 well plates at the needed cell density and further incubated for 24 hours at $37^{\circ} \mathrm{C}, 5 \% \mathrm{CO}_{2}$. 
CCD $841 \mathrm{CoN}$ was frozen and stored in liquid nitrogen following the protocol below: cells were collected using 3-4ml of trypsin EDTA for 5-15 minutes followed by treatment with $6-8 \mathrm{ml}$ of complete cell culture medium to inactivate the trypsin. Cells were then placed in $50 \mathrm{ml}$ conical centrifuge tubes and centrifuged at $125 \mathrm{x} \mathrm{g}$ for 5-10 minutes as per manufacturer recommendation. The cell pellet was re-suspended in complete growth medium with 5\% DMSO. A stepwise temperature reduction was utilized for freezing: $-20^{\circ} \mathrm{C}$ for 24 hours, $-80^{\circ} \mathrm{C}$ for an additional 24 hours, and then liquid nitrogen for long-term storage.

NCM 356 was cultured and frozen as per manufacturer recommendation. The floating cells and medium were collected and transferred to $50 \mathrm{ml}$ tubes and centrifuged at $500 \mathrm{x} \mathrm{g}$ for 5-10 min. The cell monolayer was washed with calcium and magnesium free phosphate buffered saline.

\subsubsection{Phenolic rich blueberry extract}

Northern highbush blueberries were collected from Gierke Blueberry Farm (37915 Klingville Road, Chassell, MI 49916) and immediately frozen in liquid nitrogen. For long-term storage, blueberries were placed in $-80^{\circ} \mathrm{C}$ freezer. From there, 20 grams of frozen blueberries were ground in liquid nitrogen and placed into $50 \mathrm{ml}$ VWR® SuperClear ${ }^{\mathrm{TM}}$ ultra-high performance centrifuge tubes. $10 \mathrm{~g}$ of ground blueberry was dissolved in $20 \mathrm{ml}$ of a 70/29/1 ethanol, DI water, and 3\% trifluoroacetic acid (TFA) solution. The solution was further filtered through Corning® $500 \mathrm{ml} \quad 0.22 \mu \mathrm{m}$ polyethersulfone (PES) membrane and stored in $-80^{\circ} \mathrm{C}$ freezer. $1 \mathrm{ml}$ of blueberry extract 
was centrifuged under vacuum to remove solution and then re-suspended in $1 \mathrm{ml}$ of phosphate buffered saline (PBS) and stored in $-80^{\circ} \mathrm{C}$ freezer until use on cells.

\subsubsection{Treatment of normal human colon cell lines with pro-inflammatory cytokine cocktail and blueberry extract}

Two normal colon epithelial cell lines were utilized to determine the antiinflammatory and anti-oxidant capabilities of a phenolic rich blueberry extract. In order to simulate conditions of IBD in vitro, cells were treated with a pro-inflammatory cytokine cocktail consisting of TNF- $\alpha$ (100ng/ml, Sigma), IL-1 $\beta$ (100ng/ml, Sigma), LPS $(10 \mu \mathrm{g} / \mathrm{ml}$, Sigma), and IFN- $\gamma(5 \mu \mathrm{g} / \mathrm{ml}$, Sigma) suspended in PBS for 24 hours prior to treatment with blueberry extract.

\subsubsection{Fluorescent microscopy}

NCM 356 and CCD 841 CoN were cultured as per manufacturers recommendations. Cells were seeded in triplicates in $6,12,24$, or 96 well plates in appropriate medium with 3\% heat-inactivated FBS at 25,000-100,000 cells per milliliter. Cells were imaged using either a Zeiss or Leica inverted fluorescence microscope following 24-hour treatment with a pro-inflammatory cytokine cocktail consisting of TNF- $\alpha$, IL-1 $\beta$, LPS, and IFN- $\gamma$ prior to treatment for $24-96$ hours with blueberry extract or PBS. Three fluorescent probes were used: Propidium iodide cell viability dye (ThermoFisher Scientific), CellROX ${ }^{\circledR}$ Green Reagent and CellROX ${ }^{\circledR}$ Orange Reagent for nuclear and cytoplasmic oxidative stress detection. Real time imaging was performed with propidium iodide on a Leica inverted fluorescence microscope at 1 min intervals for 
$10 \mathrm{~min}$, and then $10 \mathrm{~min}$ intervals until 1 hour. Cell fluorescence was quantified utilizing Image J software. Calculated total cell fluorescence $(\mathrm{CTCF})=$ Integrated Density $-($ Area of selected cell X Mean fluorescence of background readings). In order to compare fluorescence within and between groups, t-test, and 2-way ANOVA with Tukey's post hoc test for pairwise comparison was utilized. All values are represented as mean \pm SEM and considered significant at $\mathrm{p}<0.05$.

\subsubsection{Flow cytometry}

NCM 356 and CCD 841 CoN were seeded in 6, 12 or 24 well Nunc ${ }^{\mathrm{TM}}$ cell culture treated plates (ThermoFisher Scientific) until 70-90\% confluent. Cells were then treated with a pro-inflammatory cytokine cocktail for 24 hours prior to $24-96$ hour treatment with blueberry extract or PBS. Cells were assayed with propidium iodide for cell viability analysis under FL3, CellROX ${ }^{\circledR}$ Green Reagent and CellROX® Orange Reagent for nuclear and cytoplasmic oxidative stress detection under FL1 and FL3, respectively. Data was analyzed utilizing t-test, and 2-way ANOVA with Tukey's post hoc test for pairwise comparison. Values are represented as mean \pm SEM with significance considered at $\mathrm{p}<0.05$.

\subsection{Results}

In order to test the anti-inflammatory and antioxidant capabilities of a phenolic rich blueberry extract, we employed fluorescence microscopy and flow cytometry to 
determine their free radical scavenging capabilities and effect on cell viability following the addition of inflammatory mediators.

\subsubsection{Blueberry extract significantly reduced nuclear and cytoplasmic ROS in inflamed human colon epithelial cells based on fluorescent microscopy}

NCM 356 cells subjected to 24-hour inflammatory cocktail treatment followed by treatment with 1:20 dilution of blueberry extract for 48 hours revealed drastic decrease in CellROX ${ }^{\circledR}$ green nuclear ROS reagent in blueberry treated groups versus inflammatory control (Figure 3.1 and 3.2). Similar treatment on CCD $841 \mathrm{CoN}$ with blueberry extract showed significant decreases in CellROX ${ }^{\circledR}$ green nuclear ROS reagent fluorescence compared to inflammatory and vehicle control groups (Figure 3.7 and 3.8). NCM treatment with CellROX ${ }^{\circledR}$ orange cytoplasmic ROS reagent revealed significant decrease in cytoplasmic ROS following blueberry treatment versus both inflammatory and vehicle controls (Figure 3.3 and 3.4). Similar observation was made in CCD $841 \mathrm{CoN}$ treated with blueberry extract, which showed significant decrease in cytoplasmic ROS versus vehicle control (Figure 3.9 and 3.10). Additionally nuclear staining with Hoechst 33342 resulted in less nuclear condensation in blueberry treated groups versus all controls in NCM 356 (Figure 3.5 and 3.6) 
A.

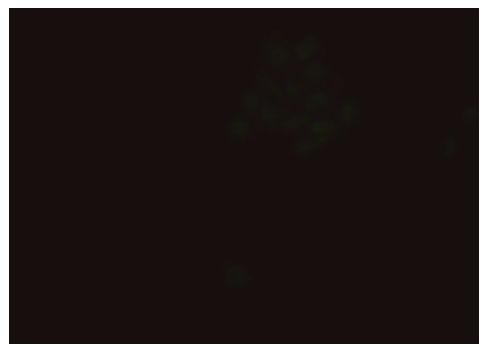

C.

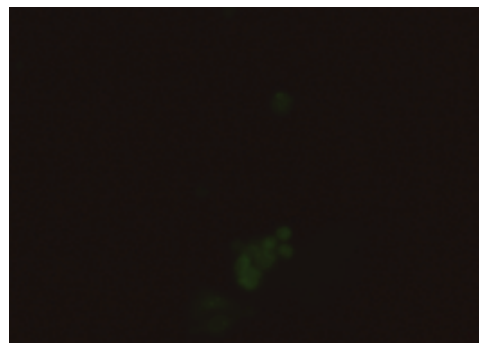

B.

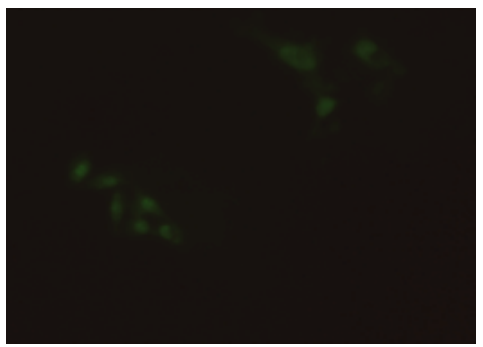

D.

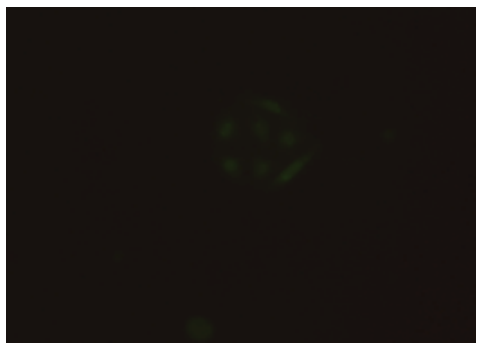

Figure 3.1: Phenolic rich blueberry extract greatly reduced nuclear ROS in NCM 356 cell line. NCM 356 cells were treated for 24 hours with the pro-inflammatory cytokine cocktail, followed by a 1:20 dilution of PBS or blueberry extract and assayed with CellROX ${ }^{\circledR}$ green nuclear ROS staining. (A) Control (B) Inflammatory control (C) 1:20 PBS control and (D) 1:20 blueberry extract. Images were taken at 20x magnification using a Zeiss inverted fluorescent microscope.

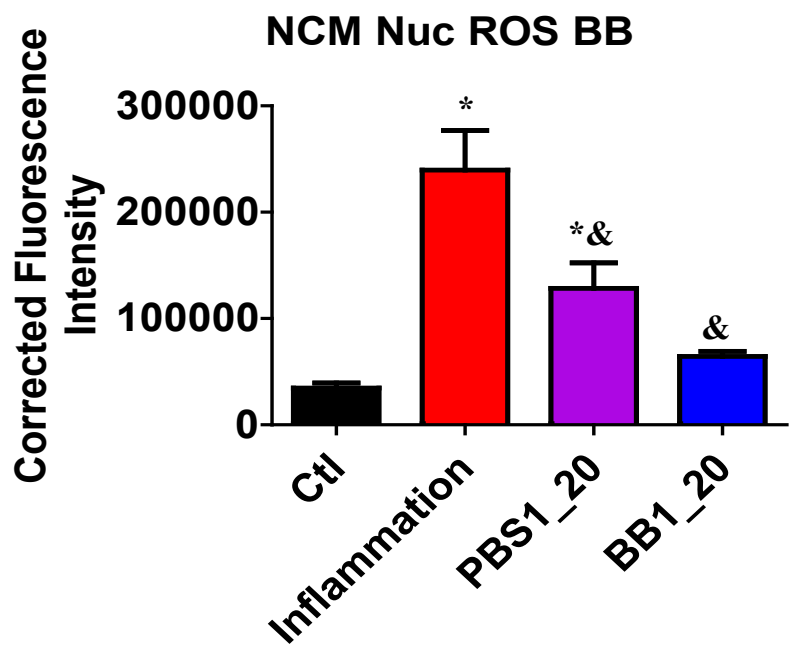


Figure 3.2 Summary data of blueberry extract nuclear ROS in NCM 356 cell line.

Quantification of CellROX ${ }^{\circledR}$ green nuclear ROS assay; all images were taken at 20x magnification using Zeiss inverted fluorescent microscope. $*=$ indicates significance vs. non treated control, $\&=$ indicates significance vs. inflammatory control, $\#=$ indicates significance vs PBS control. Results analyzed utilizing one-way ANOVA with Tukeys test, $\mathrm{n}=5 \mathrm{p}<0.05$.

A.

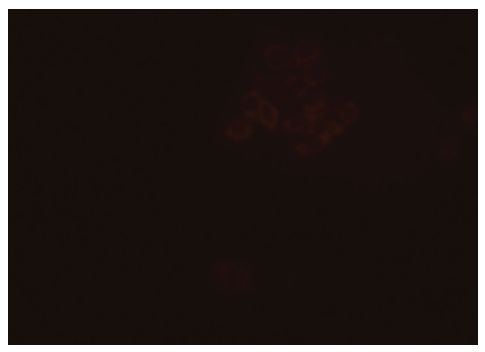

C.

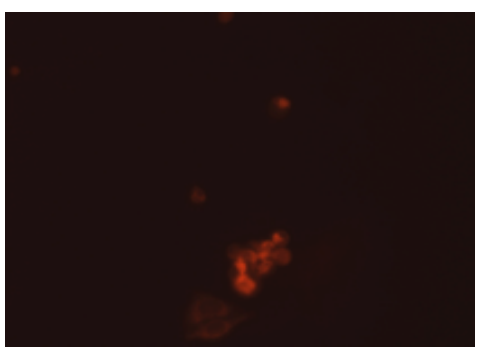

B.

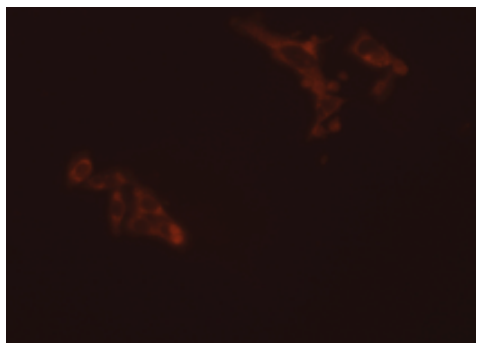

D.

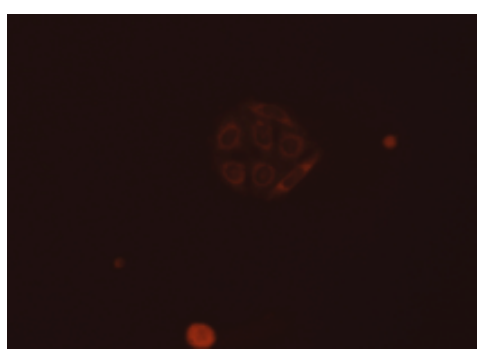

Figure 3.3: Phenolic rich blueberry extract greatly reduced cytoplasmic ROS in NCM 356 cell line. NCM 356 cells were treated for 24 hours with the pro-inflammatory cytokine cocktail, followed by a 1:20 dilution of PBS or blueberry extract and assayed with CellROX ${ }^{\circledR}$ orange cytoplasmic ROS staining. (A) Control (B) Inflammatory control (C) 1:20 PBS control and (D) 1:20 blueberry extract. Images were taken at 20x magnification using a Zeiss inverted fluorescent microscope. 


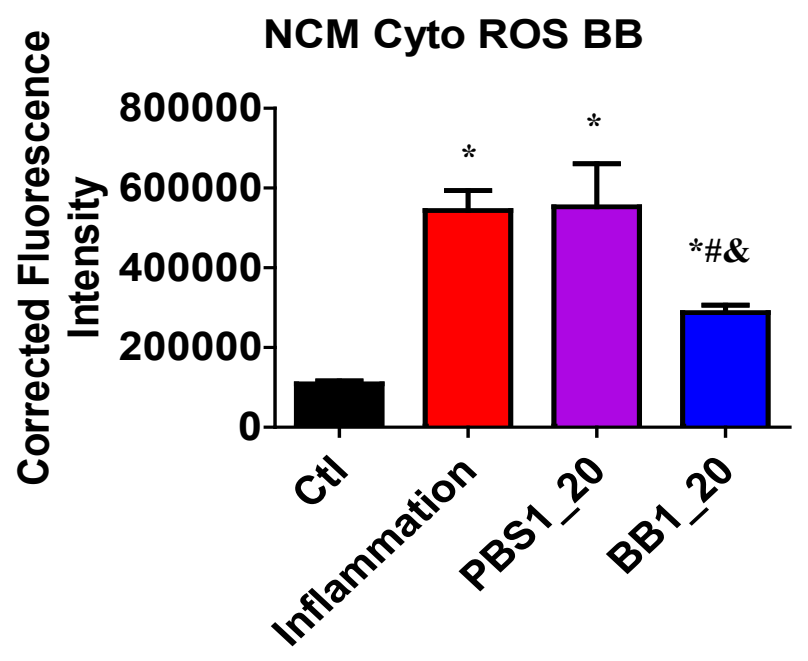

Figure 3.4 Summary data of blueberry extract cytoplasmic ROS in NCM 356 cell line. Quantification of CellROX ${ }^{\circledR}$ orange cytoplasmic ROS assay; all images were taken at 20x magnification using Zeiss inverted fluorescent microscope. $*=$ indicates significance vs. non treated control, $\&=$ indicates significance vs. inflammatory control, \#= indicates significance vs PBS control. Results analyzed utilizing one-way ANOVA with Tukeys test, $\mathrm{n}=5 \mathrm{p}<0.05$.

A.

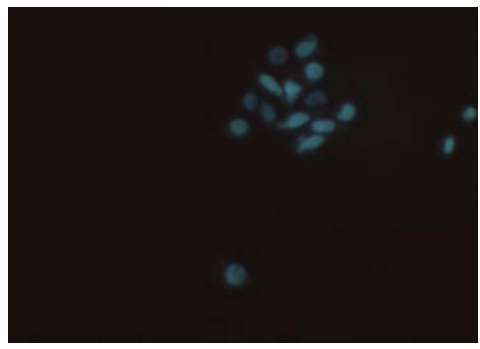

C.

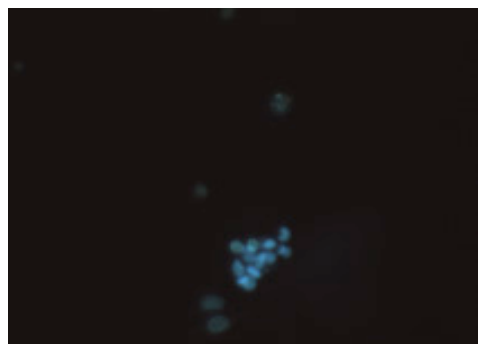

B.

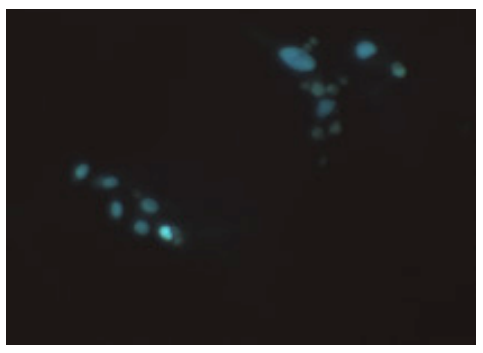

D.

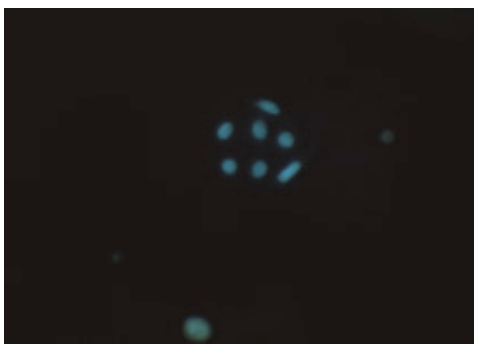


Figure 3.5: Phenolic rich blueberry extract reduced nuclear condensation in NCM 356 cell line. NCM 356 cells were treated for 24 hours with the pro-inflammatory cytokine cocktail, followed by a 1:20 dilution of PBS or blueberry extract and assayed with Hoechst 33342 nuclear stain. (A) Control (B) Inflammatory control (C) 1:20 PBS control and (D) 1:20 blueberry extract. Images were taken at 20x magnification using a Zeiss inverted fluorescent microscope.

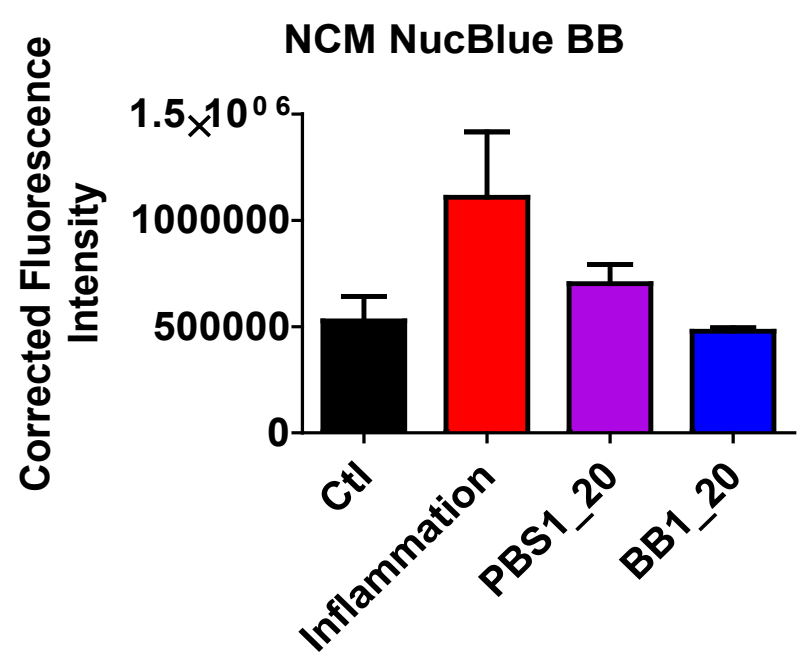

Figure 3.6 Summary data of blueberry extract nuclear condensation in NCM 356 cell line. Quantification of Hoechst 33342 nuclear staining; all images were taken at 20x magnification using Zeiss inverted fluorescent microscope, $\mathrm{n}=5$.

A.

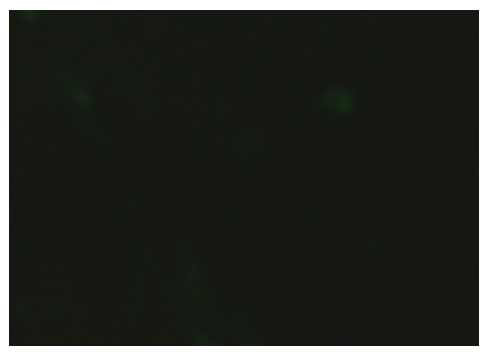

B.

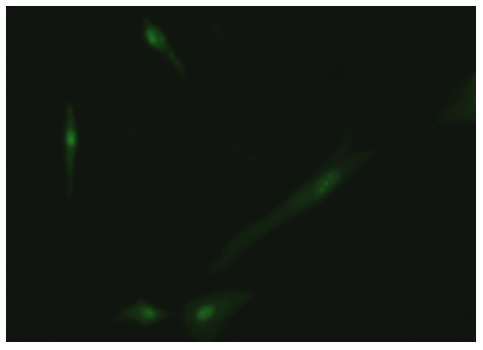


C.

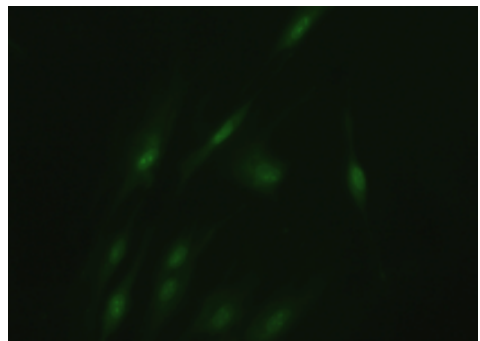

D.

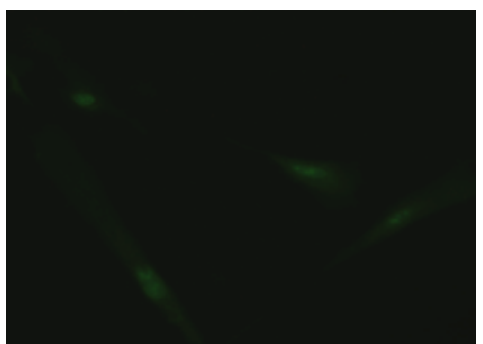

Figure 3.7: Phenolic rich blueberry extract greatly reduced nuclear ROS in CCD 841 CoN cell line. CCD $841 \mathrm{CoN}$ cells were treated for 24 hours with the proinflammatory cytokine cocktail, followed by a 1:20 dilution of PBS or blueberry extract and assayed with CellROX ${ }^{\circledR}$ green nuclear ROS staining. (A) Control (B) Inflammatory control (C) 1:20 PBS control and (D) 1:20 blueberry extract. Images were taken at 20x magnification using a Zeiss inverted fluorescent microscope.

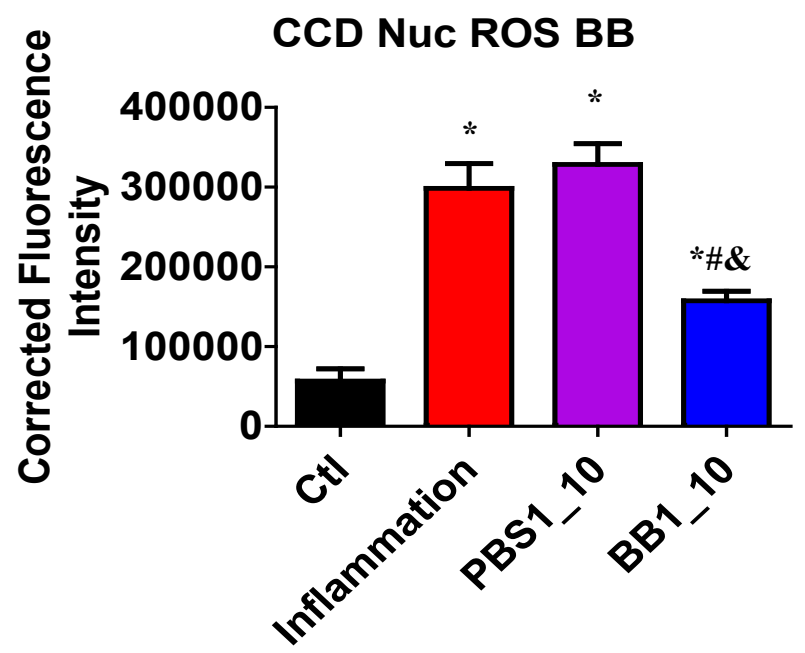

Figure 3.8 Summary data of blueberry extract nuclear ROS in CCD 841 CoN. Quantification of CellROX ${ }^{\circledR}$ green nuclear ROS assay; all images were taken at 20x magnification using Zeiss inverted fluorescent microscope. ${ }^{*}=$ indicates significance vs. non treated control, $\&=$ indicates significance vs. inflammatory control, \#= indicates 
significance vs PBS control. Results analyzed utilizing one-way ANOVA with Tukeys test, $\mathrm{n}=5 \mathrm{p}<0.05$.

A.

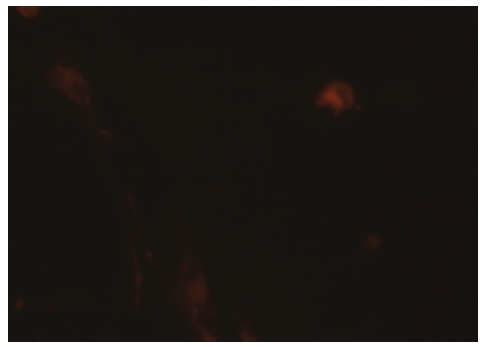

C.

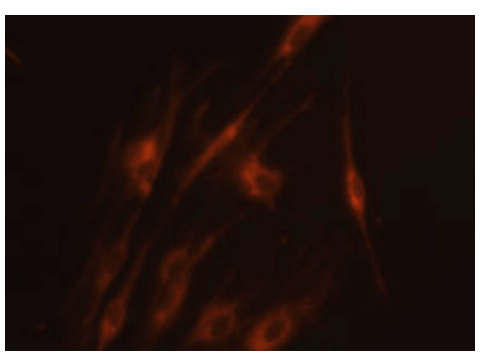

B.

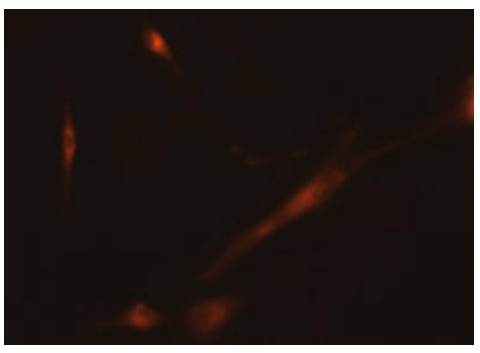

D.

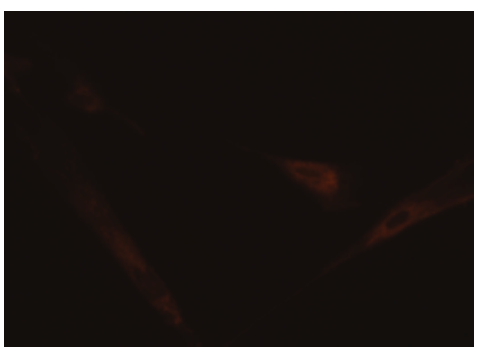

Figure 3.9: Phenolic rich blueberry extract greatly reduced cytoplasmic ROS in CCD 841 CoN cell line. CCD 841 CoN cells were treated for 24 hours with the proinflammatory cytokine cocktail, followed by a 1:20 dilution of PBS or blueberry extract and assayed with CellROX ${ }^{\circledR}$ orange cytoplasmic ROS staining. (A) Control (B) Inflammatory control (C) 1:20 PBS control and (D) 1:20 blueberry extract. Images were taken at 20x magnification using a Zeiss inverted fluorescent microscope. 


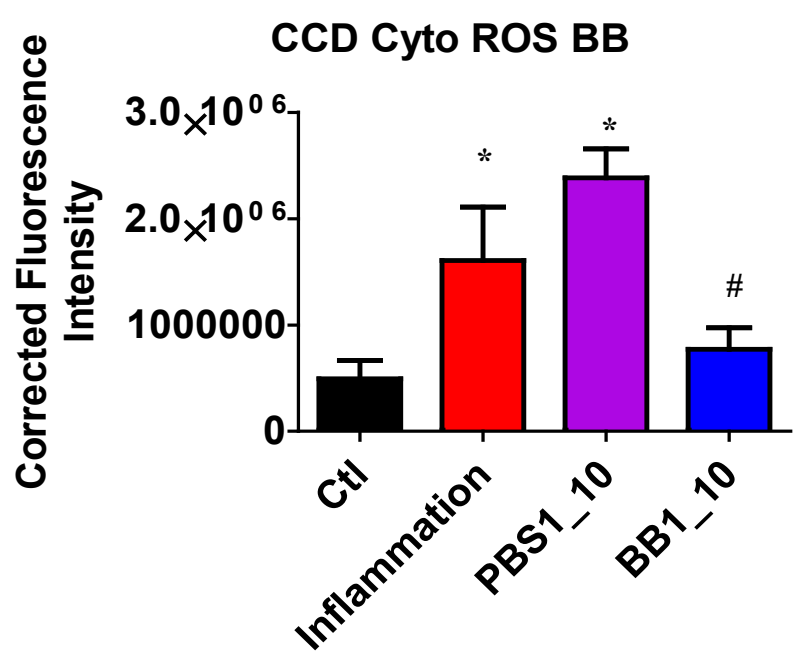

Figure 3.10 Summary data of blueberry extract cytoplasmic ROS in CCD 841 CoN cell line. Quantification of CellROX ${ }^{\circledR}$ orange cytoplasmic ROS assay; all images were taken at 20x magnification using Zeiss inverted fluorescent microscope. $*=$ indicates significance vs. non treated control, $\&=$ indicates significance vs. inflammatory control, \#= indicates significance vs PBS control. Results analyzed utilizing one-way ANOVA with Tukeys test, $\mathrm{n}=5 \mathrm{p}<0.05$.

\subsubsection{Blueberry extract significantly reduced nuclear and cytoplasmic ROS in inflamed colon epithelial cells based on flow cytometry analysis}

Results from fluorescent microscopy were verified utilizing CellROX ${ }^{\circledR}$ orange cytoplasmic and CellROX ${ }^{\circledR}$ green nuclear ROS reagent on BD Accurita C6 flow cytometer. NCM 356 treatment with 1:40 dilution of blueberry extract resulted in significantly reduction in nuclear generated ROS after 48 hours of treatment when compared to inflammatory and vehicle control groups. The cells fluorescing above 
baseline were $33.5 \%, 65.8 \%, 60.6 \%$, and $28.7 \%$ for non-treated control, inflammatory control, vehicle control and blueberry treated groups, respectively (Figure 3.11 and 3.12, $\mathrm{p}<0.05)$. Following the same conditions, blueberry extract treatment again significantly inhibited cytoplasmic generated ROS in NCM 356 with fluorescence above baseline of $25.9 \%, 60.6 \%$, and $23.1 \%$ for non-treated control, vehicle control and blueberry treated groups, respectively (Figure 3.13 and 3.14, $\mathrm{p}<0.05$ ). When NCM 356 cells are plotted against both nuclear and cytoplasmic stains, drastic reductions in cellular ROS are seen in conjunction with previous reports (Figure 3.15)

The same response was observed in CCD $841 \mathrm{CoN}$, with blueberry extract significantly reducing both cytoplasmic and nuclear generated ROS. Nuclear generated ROS was reduced from, $4.9 \%$ in non-treated control, $5.6 \%$ inflammatory control, $7.9 \%$ in inflammatory vehicle control/PBS to $3.3 \%$ above baseline following blueberry treatment (Figure 3.16 and 3.17, p<0.05). Cytoplasmic ROS was again reduced from 3.7\%, 4.4\%, and $7.6 \%$ in non-treated control, inflammatory control, and inflammatory vehicle control/PBS, respectively to $2.5 \%$ following 48 hours treatment of $1: 40$ dilution of blueberry extract (Figure 3.18 and $3.19, \mathrm{p}<0.05$ ).

A.

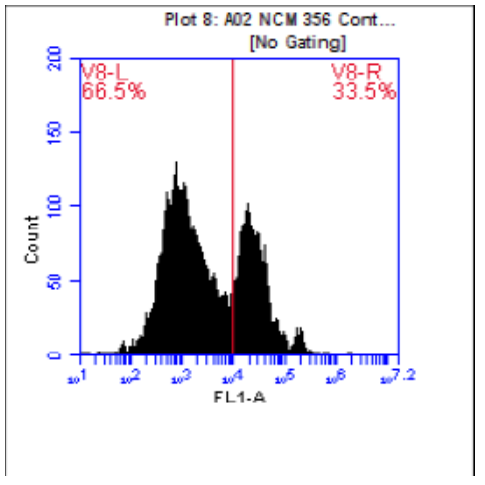

B.

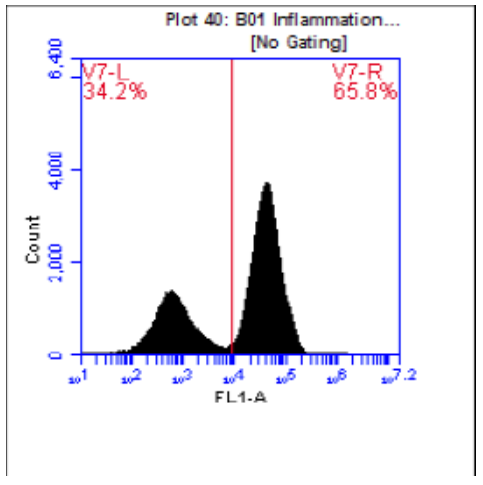


C.

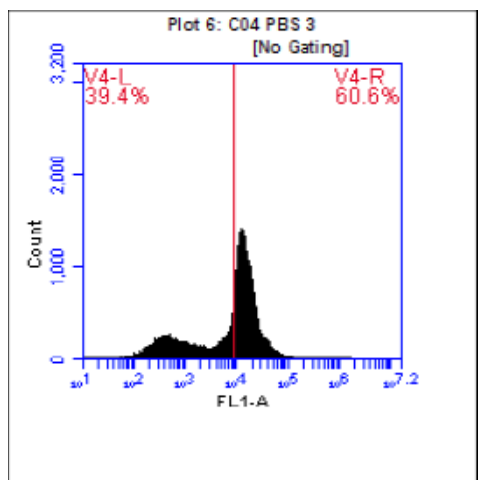

D.

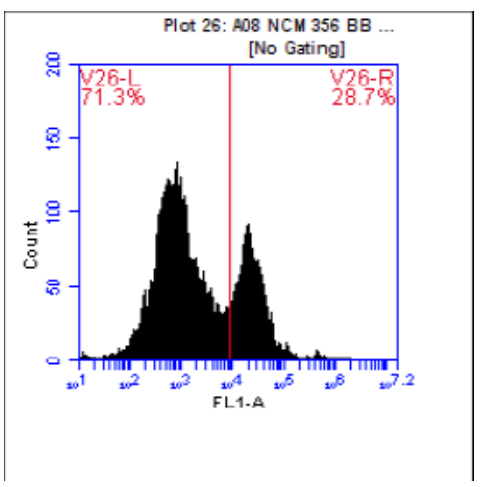

Figure 3.11: Blueberry extract decreases nuclear ROS in NCM 356 cell line as analyzed by flow cytometry. NCM 356 cells were analyzed for ROS with CellROX® green nuclear stain following 24 hour treatment with the pro inflammatory cytokine cocktail and an additional 48 hours with a 1:20 diulution of PBS or blueberry extract. (A) Control (B) Inflammatory control (C) 1:20 PBS control and (D) 1:20 blueberry extract. Cells were analyzed using an BD Accuri ${ }^{\mathrm{TM}} \mathrm{C} 6$ flow cytometer.

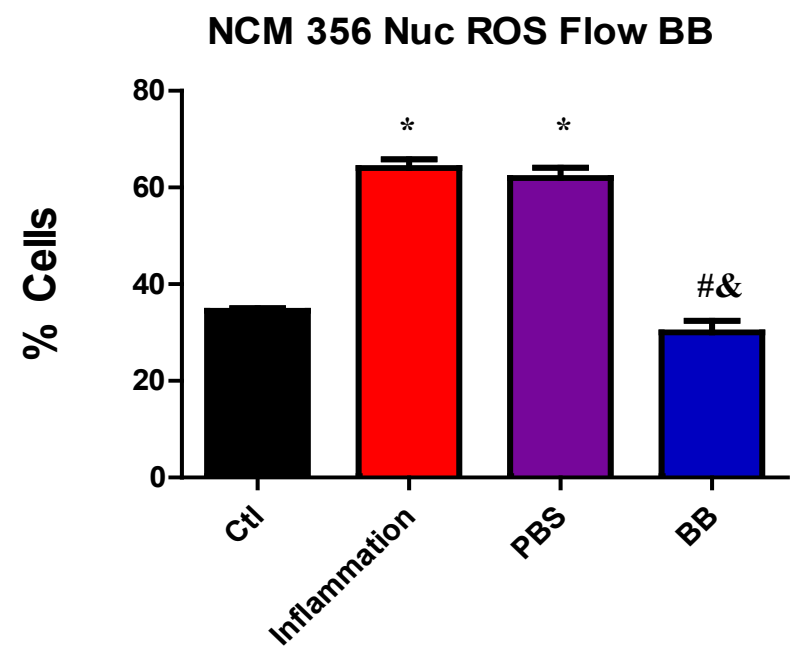

Figure 3.12: Summary data of blueberry nuclear ROS in NCM 356 as analyzed by flow cytometry. Quantification of CellROX ${ }^{\circledR}$ green nuclear ROS staining on NCM 356 
cell line. $*=$ indicates significance vs. non treated control, $\&=$ indicates significance vs. inflammatory control, \#= indicates significance vs PBS control. Results analyzed utilizing one way ANOVA with Tukeys test $(\mathrm{p}<0.05)$.

A.

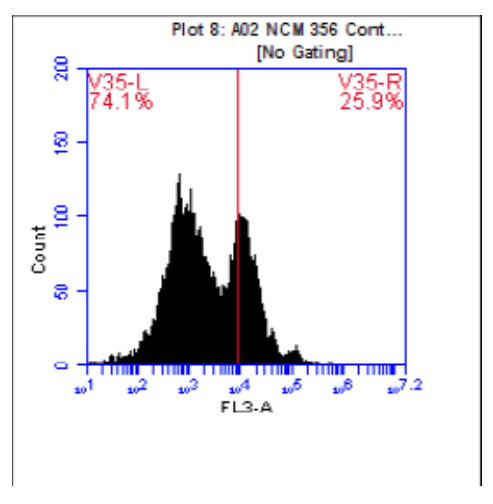

C.

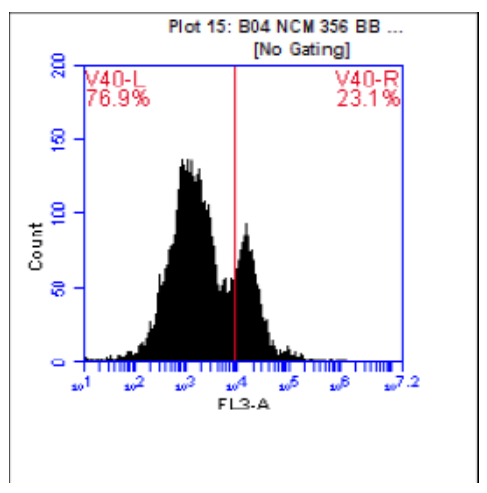

B.

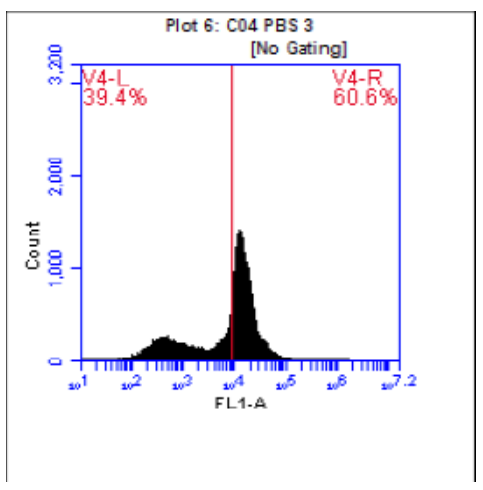

Figure 3.13: Blueberry extract decreases cytoplasmic ROS in NCM 356 cell line as analyzed by flow cytometry. NCM 356 cells were analyzed for ROS with CellROX® orange cytoplasmic stain following 24 hour treatment with the pro inflammatory cytokine cocktail and an additional 48 hours with a 1:20 diulution of PBS or blueberry extract. (A) Control (B) 1:20 PBS control and (C) 1:20 blueberry extract. Cells were analyzed using an BD Accuri ${ }^{\mathrm{TM}} \mathrm{C} 6$ flow cytometer. 
NCM 356 Cyto ROS Flow BB

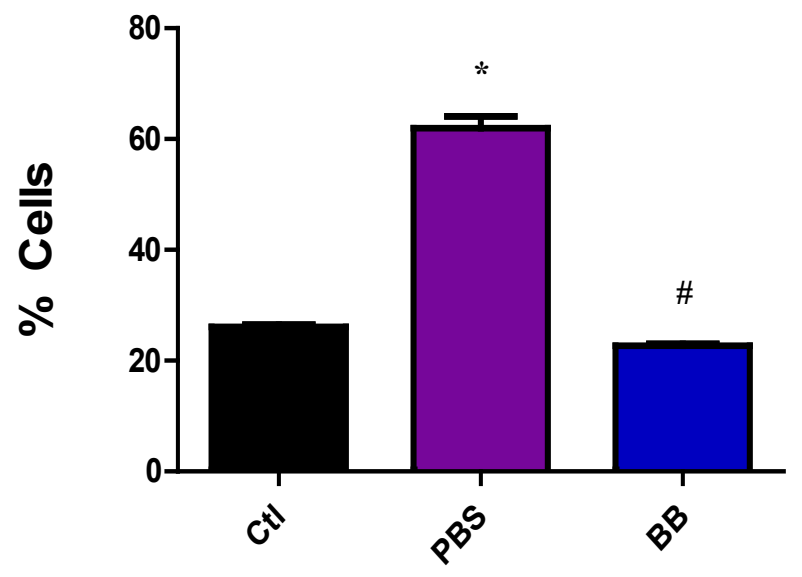

Figure 3.14: Summary data of blueberry cytoplasmic ROS in NCM 356 as analyzed by flow cytometer. Quantification of CellROX ${ }^{\circledR}$ orange cytoplasmic ROS staining on NCM 356 cell line. $*=$ indicates significance vs. non treated control, \&= indicates significance vs. inflammatory control, \#= indicates significance vs PBS control. Results analyzed utilizing one way ANOVA with Tukeys test $(\mathrm{p}<0.05)$.

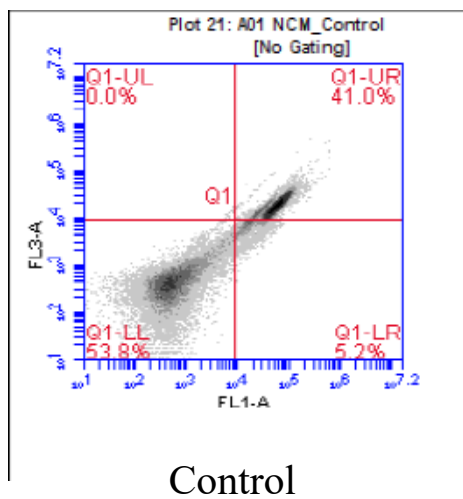

Control

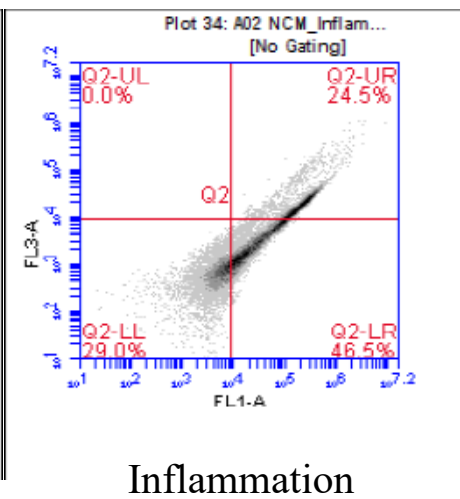

Inflammation

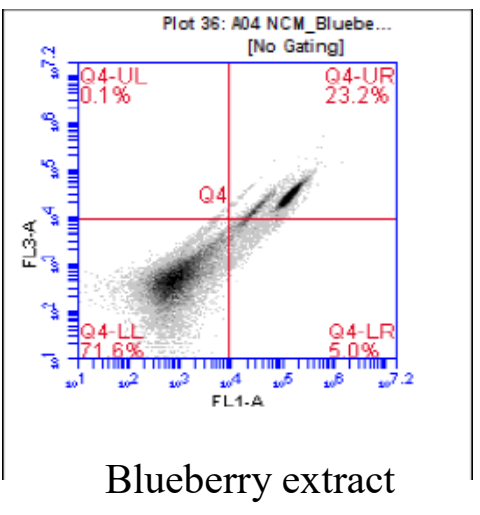

Blueberry extract

Figure 3.15: Blueberry extract decreases nuclear and cytoplasmic ROS in NCM 356 cell line as analyzed by flow cytometry. NCM 356 cells were analyzed with both CellROX ${ }^{\circledR}$ green nuclear (x-axis) and orange cytoplasmic (y-axis) stain stain following 
24 hour treatment with the pro inflammatory cytokine cocktail and an additional 48 hours with a 1:20 diulution of PBS or blueberry extract. Cells were analyzed using an BD Accuri ${ }^{\mathrm{TM}} \mathrm{C} 6$ flow cytometer.

A.

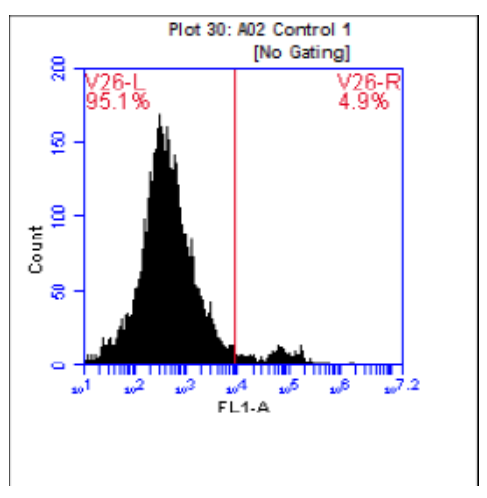

C.

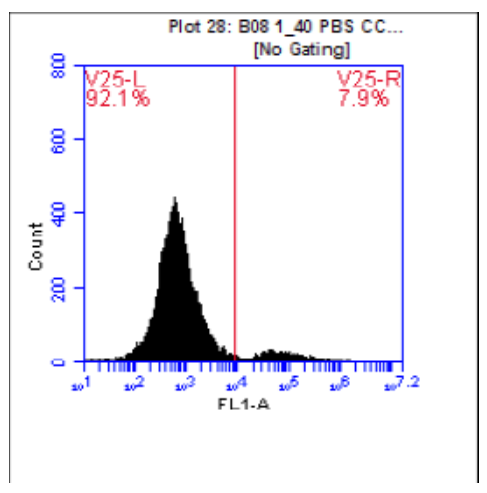

B.

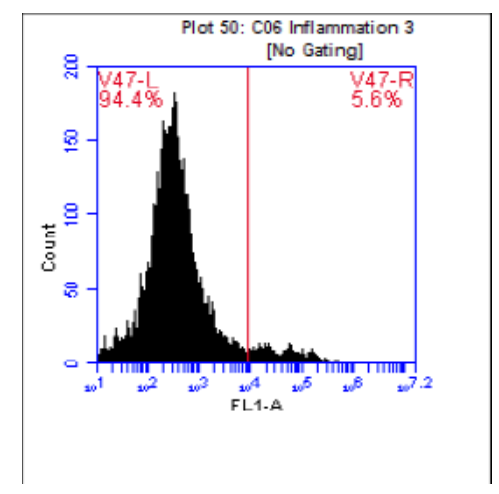

D.

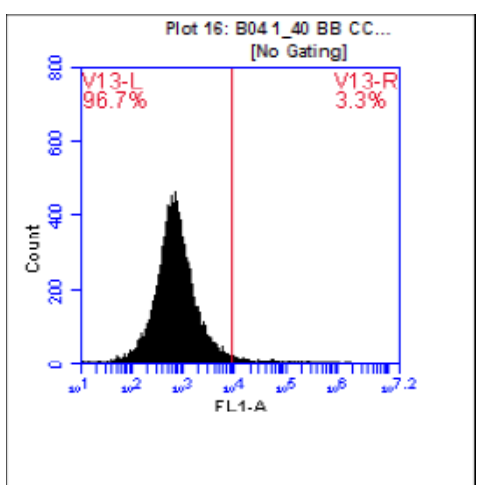

Figure 3.16: Blueberry extract decreases nuclear ROS in CCD $841 \mathrm{CoN}$ cell line as analyzed by flow cytometry. CCD $841 \mathrm{CoN}$ cells were analyzed for ROS with CellROX® green nuclear stain following 24 hour treatment with the pro inflammatory cytokine cocktail and an additional 48 hours with a 1:20 diulution of PBS or blueberry extract. (A) Control (B) Inflammatory control (C) 1:20 PBS control and (D) 1:20 
blueberry extract. Cells were analyzed using an BD Accuri ${ }^{\mathrm{TM}}$ C6 flow cytometer.

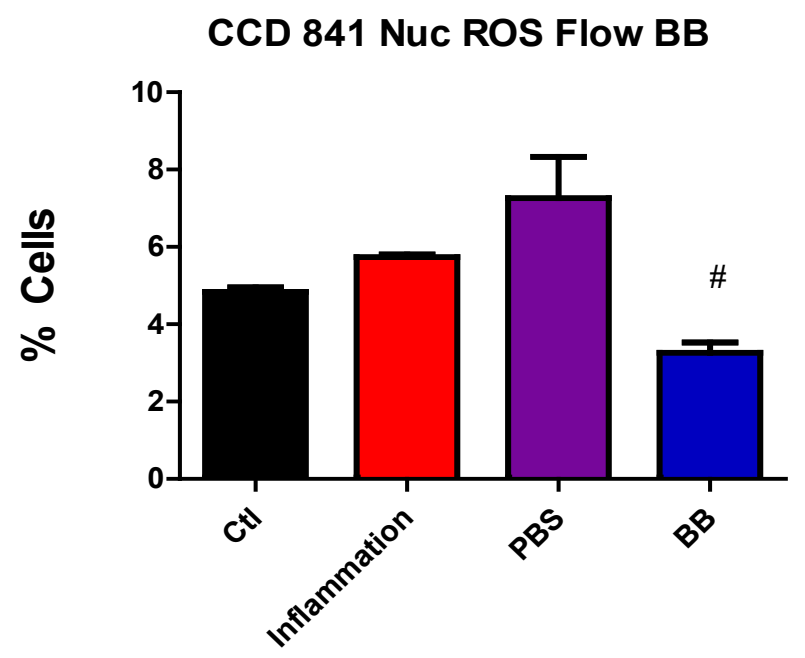

Figure 3.17: Summary data of blueberry nuclear ROS in CCD 841 CoN cell line as analyzed by flow cytometry. Quantification of CellROX ${ }^{\circledR}$ green nuclear ROS staining on CCD $841 \mathrm{CoN}$ cell line. $*=$ indicates significance vs. non treated control, $\&=$ indicates significance vs. inflammatory control, \#= indicates significance vs PBS control. Results analyzed utilizing one way ANOVA with Tukeys test $(\mathrm{p}<0.05)$.

A.

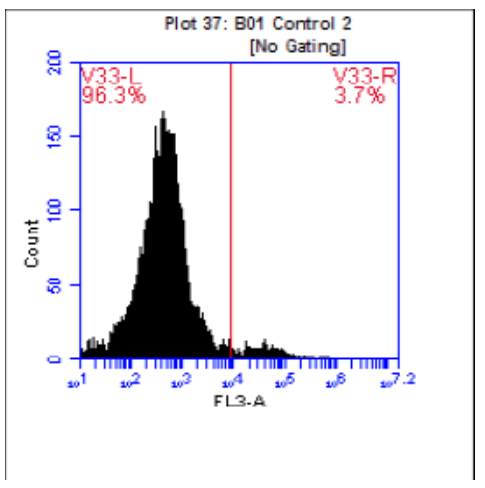

B.

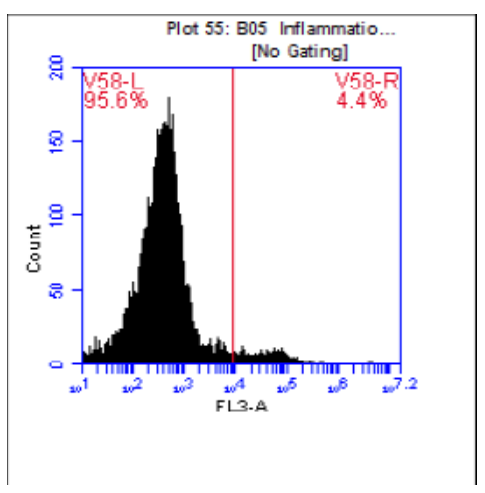


C.

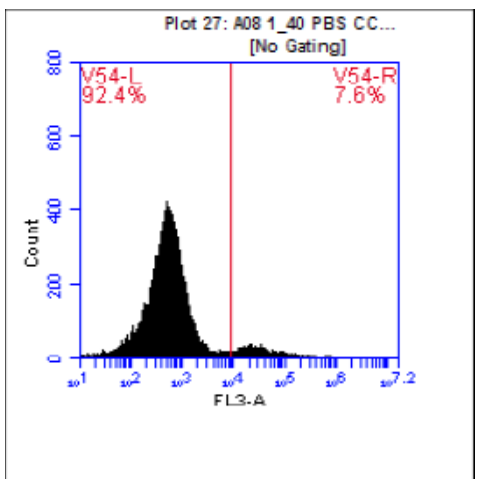

D.

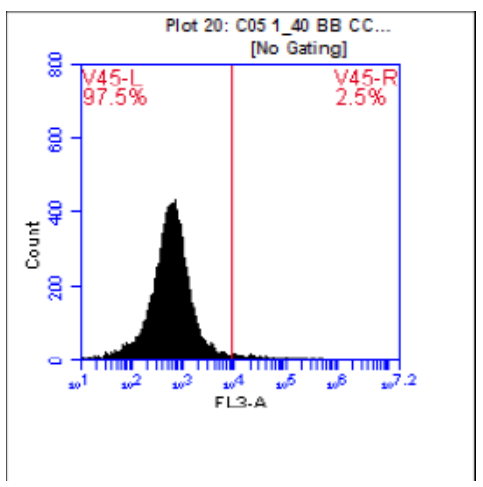

Figure 3.18: Blueberry extract decreases cytoplasmic ROS in CCD 841 CoN cell line as analyzed by flow cytometry. CCD $841 \mathrm{CoN}$ cells were analyzed for ROS with CellROX ${ }^{\circledR}$ orange cytoplasmic stain following 24 hour treatment with the pro inflammatory cytokine cocktail and an additional 48 hours with a 1:20 diulution of PBS or blueberry extract. (A) Control (B) Inflammatory control (C) 1:20 PBS control and (D) 1:20 blueberry extract. Cells were analyzed using an BD Accuri' ${ }^{\mathrm{TM}}$ C6 flow cytometer.

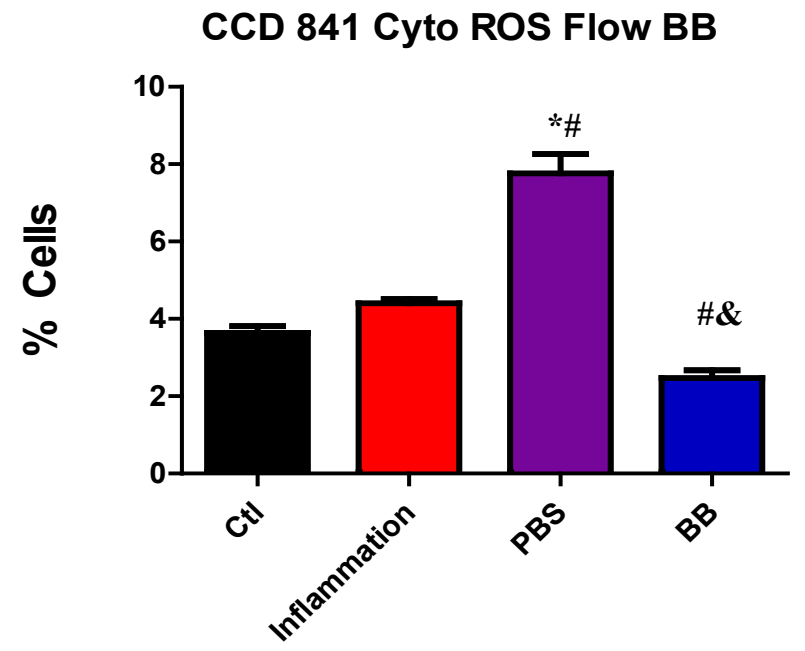

Figure 3.19: Summary data of blueberry extract cytoplasmic ROS in CCD 841 CoN cell line as analyzed by flow cytometry. Quantification of CellROX ${ }^{\circledR}$ orange 
cytoplasmic ROS staining on CCD $841 \mathrm{CoN}$ cell line. ${ }^{*}=$ indicates significance vs. non treated control, $\&=$ indicates significance vs. inflammatory control, \#= indicates significance vs PBS control. Results analyzed utilizing one way ANOVA with Tukeys test $(\mathrm{p}<0.05)$.

\subsubsection{Bluberry Exract Significantly reduced cell death following treatment with the pro-inflammatory cytokine cocktail based on flow cytometry analysis}

CCD 841 CoN cells were assayed for viability utilizing propidium iodide staining following treatment with the pro-inflammatory cytokine cocktail and blueberry extract. Non-treated control cells showed $16.5 \%$ of the population not being viable compared to $20.5 \%$ following treatment with the pro-inflammatory cytokine cocktail, whereas blueberry extract treatment reduced cell death to $11.6 \%$ (Figure 3.20 and $3.21, \mathrm{p}<0.05$ ).

A.

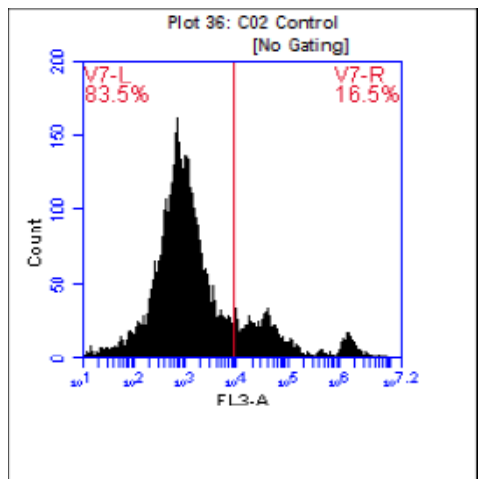

B.

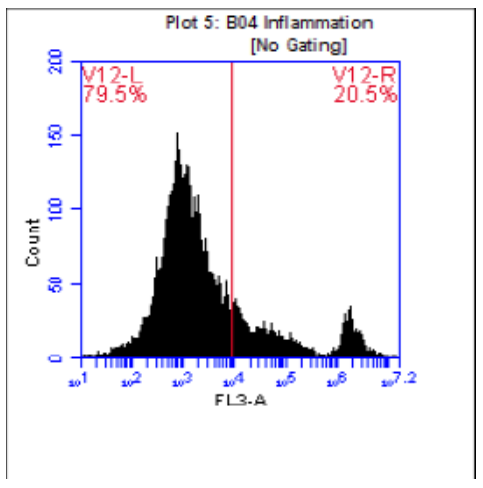


C.

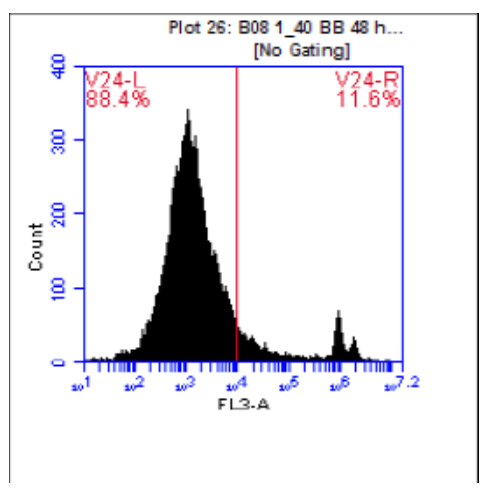

Figure 3.20 Blueberry extract reduced the effects of the pro-inflammatory cytokine cocktail on cell death in CCD 841 CoN cell line. CCD 841 CoN were treated for 24 hours with the pro-inflammatory cytokine cocktail followed by 48 hours of a 1:20 dilution of blueberry extract and then assayed with propidium iodide cell viability assay. (A) Control (B) Inflammatory control and (C) 1:40 blueberry extract.

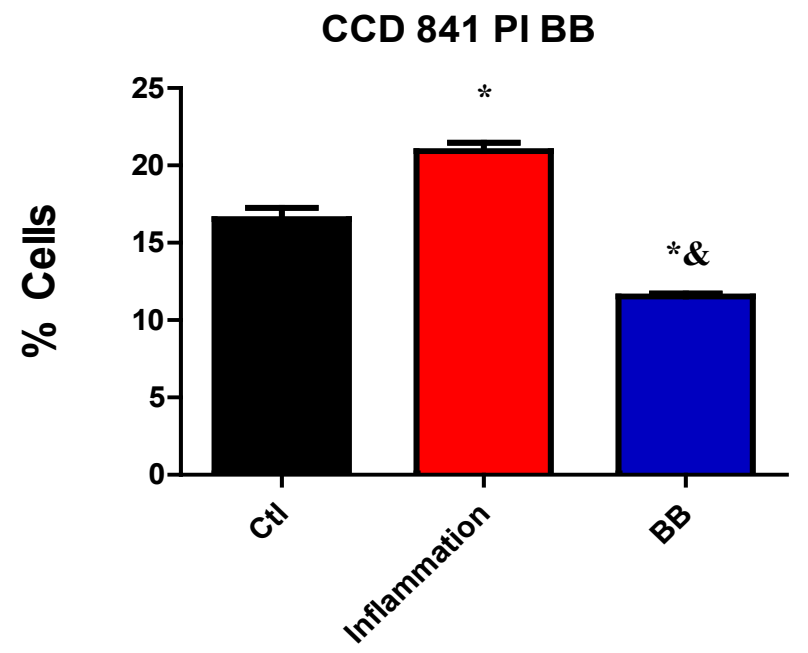

Figure 3.21: Summary data of reduced cell death by blueberry extract on CCD 841 CoN. Quantification of propidium iodide cell viablity assay on CCD $841 \mathrm{CoN}$ cell line, *= indicates significance vs. non treated control, $\&=$ indicates significance vs. 
inflammatory control, \#= indicates significance vs PBS control. Results analyzed utilizing one way ANOVA with Tukeys test $(\mathrm{p}<0.05)$.

\subsection{Discussion}

Free radicals are increasingly being associated with the occurrence and progression of chronic diseases in humans ${ }^{87}$. ROS generation and elimination play a critical role in maintenance of cellular homeostasis with high oxidative stress attributed to cancer, aging, and neurodegeneration ${ }^{36,37}$. ROS is produced from oxygen and includes several species such as superoxide and the hydroxyl radical. ROS is produced intrinsically from normal cellular metabolism. Additionally, it is produced from several different cell types such as polymorphonuclear neutrophils, as well as others involved in host-defense responses ${ }^{36,37}$. These species play a crucial role in regulating cellular functions, such as cell proliferation by oxidizing signaling proteins. Thus, these attributes make ROS very important in understanding processes involved in aging, inflammation and cancer.

Treatment of both NCM 356 and CCD 841 CoN with pro-inflammatory cytokine cocktail resulted in increase in both cytoplasmic and nuclear generated ROS. The cytokine TNF- $\alpha$ has been shown to induce ROS in several different cell systems, such as hepatocytes, rat glomeruli, HeLa and HEK 293 cells among others ${ }^{88-90}$. Additionally, a plethora of research suggests that both IL-1 $\beta$ and LPS induce ROS ${ }^{91-93}$. For example, IL$1 \beta$ was shown to increase ROS production in TMK-1 cells through IL-8 and MAP kinase dependent mechanisms ${ }^{94}$. LPS induced the production of ROS resulting in the activation 
of TRAF6-ASK1-p38 signaling mechanisms, which are crucial for innate immunity in ASK1-deficient mice ${ }^{91}$. These studies show the role played by ROS in cell signaling cascades as well as various inflammatory processes. Treatment of both NCM 356 and CCD $841 \mathrm{CoN}$ with the phenolic rich blueberry extract was able to greatly reduce nuclear and cytoplasmic ROS. The mechanisms by which blueberry fractions work vary in various cell systems. Blueberry polyphenols inhibit inflammatory responses in brain microglial cells by regulating several inflammatory cytokines, TNF- $\alpha$, IL-1 $\beta$, LPS, and nitric oxide ${ }^{95}$. Another study showed blueberry extract to be effective in inhibiting lipid peroxidation in a liposomal membrane system ${ }^{96}$.

Several different compounds have been identified in blueberry extracts, with the most abundant being delphinidin, malvidin, and cyanidin, which exhibit strong antiinflammatory and anti-tumorigenic effects. Delphinidin is an anthocyanidin found not only in blueberries but also in concord grapes, cranberries, and pomegranates. Delphinidin acts through a wide range of pathways such as caspase activation, cell cycle arrest, cytokine inhibition among others ${ }^{97-99}$. Delphinidin inhibited Interleukin 1 beta (IL$1 \beta$ ) induced activation of NF- $\mathrm{KB}$, and COX-2 expression in human chondrocytes. IL-1 $\beta$ and NF- $\mathrm{kB}$ are major mediators of inflammation and disease progression ${ }^{97}$. Consumption of red wine extract, which included delphinidin, reduced monocyte chemo-attractant protein 1 (MCP-1) levels and enhanced antioxidant activity in blood plasma ${ }^{99}$. Another study found that the combination of cyanidin, malvidin, peonidin, petunidin and delphinidin suppressed the growth of aggressive non-small-cell lung cancer with very little effect on normal cells by inducing cell cycle arrest and apoptosis mediated by 
oncogenic notch and WNT pathways as well as their downstream targets ${ }^{100}$. Delphinidin also showed positive results with regards to inflammation of human airway epithelial cells (NCI-H292) ${ }^{98}$. Another study found delphinidin to induce apoptosis in human colon carcinoma cells (HCT116) and human leukemia cells (HL60) ${ }^{101}$. Delphinidin was shown to downregulate the expression of matrix metalloproteinase 9 in MCF-7 human breast cancer cell line following inflammatory induction by phorbol 12-myristate 13 -acetate ${ }^{102}$. This metalloproteinase has a role in metastasis mediated through NF- $\mathrm{kB}$.

Malvidin is another anthocyanidin found in berries, such as blueberries and cranberries. It also exhibits antioxidants, chemo-preventative, and cardio protective properties ${ }^{103}$. Malvidin drastically decreased the expression of TNF- $\alpha$, IL1, IL-6 and iNOS in human peripheral blood mononuclear cells $(\mathrm{PBMC})^{104}$. Macrophages stimulated by bacterial LPS, when treated with malvidin, decreased expression of NF- $\mathrm{BB}$ and ROS production and upregulated levels of Akt and MAPK phosphatase- $1^{105}$.

Cyanidin, more specifically, cyanidin-3-O- $\beta$-D-glucoside $(\mathrm{C} 3 \mathrm{G})$ is a watersoluble anthocyanin pigment with antioxidant, anti-carcinogenic, and anti-inflammatory properties $^{106,107}$. For example, in human THP-1 cells, C3G inhibited the expression of IL6 and TNF- $\alpha$ in LPS stimulated inflammatory model ${ }^{107}$. Additionally, C3G showed strong anti-inflammatory properties in HT-29 cell line treated with a pro-inflammatory cytokine cocktail consisting of IL-1 $\alpha$, TNF- $\alpha$, and IFN- $\gamma$ which reduced the expression of NO, PGE 2 IL-8, iNOS, and COX-2 at lower concentrations than the NSAID, 5aminosalicyclic acid ${ }^{108}$. This study suggests that anthocyanins have the ability to reach the gastrointestinal tract and exhibit potent anti-inflammatory effects ${ }^{108}$. C3G inhibited 
UVB-induced skin damage in mice by reducing glutathione depletion and lipid peroxidation as well as IL- 6 , and TNF- $\alpha$, which are associated with inflammatory $\operatorname{processes}^{109}$.

The anti-inflammatory effects, based on decrease in ROS, in cell lines treated with blueberry extract is likely due to the anthocyanidins present in blueberry extract. Furthermore, our research group examined the comparative effects of novel rice callus suspension culture (RCSC) against blueberry extract in free radical scavenging capabilities. In comparing the two, blueberry extract was greatly more efficient at decreasing nuclear and cytoplasmic ROS versus RCSC. However, RCSC was better at preventing cytokine induced cell death as analyzed by flow cytometry with propidium iodide staining. Furthermore, while a plethora of literature exists on the benefits of maintenance of ROS, in general decreases have shown to be beneficial to cell systems.

Increases in nuclear and cytoplasmic ROS could be a beneficial response to the pro-inflammatory cytokines by disrupting cell-signaling pathways that trigger cell death through oxidation of key molecular targets, thus increasing cell viability. This was seen following treatment with RCSC. On the other hand, since the pro-inflammatory cytokine cocktail increases oxidative stress, a mechanism that often leads to cell death, decreasing oxidative stress by blueberry extract could additionally be a mechanism of survival. 


\subsection{Conclusion and future work}

Blueberry extract exhibited strong anti-inflammatory and anti-oxidant capabilities following treatments of two colon epithelial cell lines with a pro-inflammatory cytokine cocktail followed by cytoplasmic and nuclear ROS assays. These results align with previous research as well as the importance of consuming antioxidant rich foods. While several compounds have been identified in blueberry extracts, only a few are described above. Some of these compounds have been implicated as possible drug candidates for inflammatory conditions such as IBD where maintenance of redox homeostasis is of crucial importance. Further research is needed to fully understand their effects in vivo. Development of blueberry callus suspension culture and its use as an anti-inflammatory agent is another area of future research. If blueberry callus suspension culture works as a better anti-inflammatory agent than blueberry extract, novel drugs can be developed with little or no side effects. The chemical constituents of blueberry callus suspension culture can be identified and compared with that of blueberry extract and the biochemical pathways and molecular mechanisms employed need to be investigated.

\subsection{Acknowledgements}

This work was supported by the National Institute of Food and Agriculture, U.S. Department of Agriculture, under award number 2014-67018-21767. 


\section{References}

1 Croteau, R., Kutchan, T. M. \& Lewis, N. G. Natural products (secondary metabolites). Biochemistry and Molecular Biology of Plants 24, 1250-1319 (2000).

2 Bourgaud, F., Gravot, A., Milesi, S. \& Gontier, E. Production of plant secondary metabolites: a historical perspective. Plant Science 161, 839-851, doi:https://doi.org/10.1016/S0168-9452(01)00490-3 (2001).

3 Cragg, G. M. \& Newman, D. J. Natural products: a continuing source of novel drug leads. Biochimica et Biophysica Acta (BBA)-General Subjects 1830, 36703695 (2013).

4 Newman, D. J. \& Cragg, G. M. Natural products as sources of new drugs over the 30 years from 1981 to 2010. Journal of Natural Products 75, 311-335, doi:10.1021/np200906s (2012).

5 Ramakrishna, A. \& Ravishankar, G. A. Influence of abiotic stress signals on secondary metabolites in plants. Plant Signaling \& Behavior 6, 1720-1731, doi:10.4161/psb.6.11.17613 (2011).

6 Herrmann, K. M. \& Weaver, L. M. THE SHIKIMATE PATHWAY. Annual Review of Plant Physiology and Plant Molecular Biology 50, 473-503, doi:10.1146/annurev.arplant.50.1.473 (1999).

7 Abdallah, I. I. \& Quax, W. J. A Glimpse into the Biosynthesis of Terpenoids. KnE Life Sciences 3, 81-98 (2017).

8 Cordell, G. A., Quinn- Beattie, M. L. \& Farnsworth, N. R. The potential of alkaloids in drug discovery. Phytotherapy Research 15, 183-205 (2001).

9 Rathee, P. et al. Mechanism of action of flavonoids as anti-inflammatory agents: a review. Inflammation \& Allergy-Drug Targets (Formerly Current Drug Targets-Inflammation \& Allergy) 8, 229-235 (2009).

10 Badri, D. V. \& Vivanco, J. M. Regulation and function of root exudates. Plant, Cell \& Environment 32, 666-681 (2009).

11 Cai, Z., Kastell, A., Knorr, D. \& Smetanska, I. Exudation: an expanding technique for continuous production and release of secondary metabolites from plant cell suspension and hairy root cultures. Plant Cell Reports 31, 461477 (2012).

12 Rahman, N., Dhadi, S. R., Deshpande, A. \& Ramakrishna, W. Rice callus suspension culture inhibits growth of cell lines of multiple cancer types and induces apoptosis in lung cancer cell line. BMC Complementary and Alternative Medicine 16, 427, doi:10.1186/s12906-016-1423-3 (2016).

13 Deshpande, A., Dhadi, S. R., Hager, E. J. \& Ramakrishna, W. Anticancer Activity of Rice Callus Suspension Culture. Phytotherapy Research 26, 1075-1081, doi:10.1002/ptr.3699 (2012).

14 Hanauer, S. B. Inflammatory bowel disease: epidemiology, pathogenesis, and therapeutic opportunities. Inflammatory Bowel Diseases 12 (2006). 
15 Podolsky, D. K. Inflammatory bowel disease. New England Journal of Medicine 325, 1008-1016 (1991).

16 Neurath, M. F. Cytokines in inflammatory bowel disease. Nature Reviews. Immunology 14, 329 (2014).

17 Baumgart, D. Crohn's Disease and Ulcerative Colitis. (Springer, 2011).

18 Xavier, R. \& Podolsky, D. Unravelling the pathogenesis of inflammatory bowel disease. Nature 448, 427-434 (2007).

19 Lichtenstein, G. R., Hanauer, S. B. \& Sandborn, W. J. Management of Crohn's disease in adults. The American Journal of Gastroenterology 104, 465 (2009).

20 Neurath, M. F. Cytokines in inflammatory bowel disease. Nature Reviews Immunology 14, 329-342 (2014).

21 Engel, M. A. \& Neurath, M. F. New pathophysiological insights and modern treatment of IBD. Journal of Gastroenterology 45, 571-583 (2010).

22 Harvey, A. L. Natural products in drug discovery. Drug Discovery Today 13, 894-901, doi:10.1016/j.drudis.2008.07.004 (2008).

23 Patridge, E., Gareiss, P., Kinch, M. S. \& Hoyer, D. An analysis of FDA-approved drugs: natural products and their derivatives. Drug Discovery Today 21, 204207, doi:10.1016/j.drudis.2015.01.009 (2016).

24 Furst, R. \& Zundorf, I. Plant-derived anti-inflammatory compounds: hopes and disappointments regarding the translation of preclinical knowledge into clinical progress. Mediators of Inflammation 2014, 146832, doi:10.1155/2014/146832 (2014).

25 Gonzalez, R. et al. Effects of flavonoids and other polyphenols on inflammation. Critical Reviews in Food Science and Nutrition 51, 331-362, doi:10.1080/10408390903584094 (2011).

26 Kennedy, D. O. \& Wightman, E. L. Herbal Extracts and Phytochemicals: Plant Secondary Metabolites and the Enhancement of Human Brain Function. Advances in Nutrition 2, 32-50, doi:10.3945/an.110.000117 (2011).

27 Rhodes, M. J. C. Physiological roles for secondary metabolites in plants: some progress, many outstanding problems. Plant Molecular Biology 24, 1-20, doi:10.1007/bf00040570 (1994).

28 Tahara, S. A journey of twenty-five years through the ecological biochemistry of flavonoids. Bioscience, Biotechnology, and Biochemistry 71, 1387-1404, doi:10.1271/bbb.70028 (2007).

29 Crozier, A., Clifford, M. N. \& Ashihara, H. Plant secondary metabolites: occurrence, structure and role in the human diet. (John Wiley \& Sons, 2008).

30 Zwenger, S. Plant terpenoids: applications and future potentials. Biotechnology and Molecular Biology Reviews 3, 1-7 (2008).

31 de Sousa Falcao, H. et al. Gastric and duodenal antiulcer activity of alkaloids: a review. Molecules (Basel, Switzerland) 13, 3198-3223, doi:10.3390/molecules13123198 (2008).

32 Arumugam, S., Kavimani, S., Kadalmani, B., Ahmed, B. A. \& Akbarsha, M. A. Antidiabetic activity of leaf and callus extracts of Aegle marmelos in rabbit. Sci Asia 34, doi:10.2306/scienceasia1513-1874.2008.34.317 (2008). 
33 Sohn, K. J. et al. Molecular genetics of ulcerative colitis-associated colon cancer in the interleukin 2- and beta(2)-microglobulin-deficient mouse. Cancer Research 61, 6912-6917 (2001).

34 Shacter, E. \& Weitzman, S. A. Chronic inflammation and cancer. Oncology 16, 217-232 (2002).

35 Eiró, N. \& Vizoso, F. J. Inflammation and cancer. World Journal of Gastrointestinal Surgery 4, 62-72, doi:10.4240/wjgs.v4.i3.62 (2012).

36 Trachootham, D., Lu, W., Ogasawara, M. A., Nilsa, R. D. \& Huang, P. Redox regulation of cell survival. Antioxidants \& Redox Signaling 10, 1343-1374, doi:10.1089/ars.2007.1957 (2008).

37 Mittal, M., Siddiqui, M. R., Tran, K., Reddy, S. P. \& Malik, A. B. Reactive oxygen species in inflammation and tissue injury. Antioxidants \& Redox Signaling 20, 1126-1167 (2014).

38 Elmore, S. Apoptosis: a review of programmed cell death. Toxicologic Pathology 35, 495-516 (2007).

39 Orrenius, S., Gogvadze, V. \& Zhivotovsky, B. Calcium and mitochondria in the regulation of cell death. Biochemical and Biophysical Research

Communications 460, 72-81 (2015).

40 Chao, C. et al. CCK2 receptor expression transforms non-tumorigenic human NCM356 colonic epithelial cells into tumor forming cells. International Journal of Cancer. Journal International du Cancer 126, 864-875, doi:10.1002/ijc.24845 (2010).

41 Morgan, M. J., Kim, Y. S. \& Liu, Z. G. TNFalpha and reactive oxygen species in necrotic cell death. Cell Research 18, 343-349, doi:10.1038/cr.2008.31 (2008).

42 Shen, H. M. \& Pervaiz, S. TNF receptor superfamily-induced cell death: redoxdependent execution. FASEB journal : Official Publication of the Federation of American Societies for Experimental Biology 20, 1589-1598, doi:10.1096/fj.05-5603rev (2006).

43 Dignass, A. U. \& Podolsky, D. K. Interleukin 2 modulates intestinal epithelial cell function in vitro. Experimental Cell Research 225, 422-429, doi:10.1006/excr.1996.0193 (1996).

44 Rauch, I., Muller, M. \& Decker, T. The regulation of inflammation by interferons and their STATs. Jak-stat 2, e23820, doi:10.4161/jkst.23820 (2013).

45 Morgan, M. J. \& Liu, Z.-g. Crosstalk of reactive oxygen species and NF-кB signaling. Cell Research 21, 103-115 (2011).

46 Rotondi, M., Lazzeri, E., Romagnani, P. \& Serio, M. Role for interferon- $\gamma$ inducible chemokines in endocrine autoimmunity: an expanding field. Journal of Endocrinological Investigation 26, 177-180 (2003).

47 Ghosh, S., Chaudhary, R., Carpani, M. \& Playford, R. Interfering with interferons in inflammatory bowel disease. Gut 55, 1071-1073 (2006).

48 Tsukada, Y., Nakamura, T., Iimura, M., Iizuka, B.-e. \& Hayashi, N. Cytokine profile in colonic mucosa of ulcerative colitis correlates with disease activity 
and response to granulocytapheresis. The American Journal of Gastroenterology 97, 2820-2828 (2002).

49 Hanai, H. et al. Correlation of serum soluble TNF- $\alpha$ receptors I and II levels with disease activity in patients with ulcerative colitis. The American Journal of Gastroenterology 99, 1532-1538 (2004).

50 Wallach, D. et al. Tumor necrosis factor receptor and Fas signaling mechanisms. Annual Review of Immunology 17, 331-367 (1999).

51 Francescone, R., Hou, V. \& Grivennikov, S. I. Cytokines, IBD, and colitisassociated cancer. Inflammatory Bowel Diseases 21, 409-418 (2015).

52 Cseke, L. J. et al. Natural Products From Flants. (CRC press, 2016).

53 Azmir, J. et al. Techniques for extraction of bioactive compounds from plant materials: a review. Journal of Food Engineering 117, 426-436 (2013).

54 Hernández, Y., Lobo, M. G. \& González, M. Factors affecting sample extraction in the liquid chromatographic determination of organic acids in papaya and pineapple. Food Chemistry 114, 734-741 (2009).

55 Ananthakrishnan, A. N. in Inflammatory Bowel Disease: Diagnosis and Therapeutics (ed Russell D. Cohen) 1-11 (Springer International Publishing, 2017).

56 Hadjipetrou, A., Anyfantakis, D., Galanakis, C. G., Kastanakis, M. \& Kastanakis, S. Colorectal cancer, screening and primary care: A mini literature review. World Journal of Gastroenterology 23, 6049-6058, doi:10.3748/wjg.v23.i33.6049 (2017).

57 Schieber, M. \& Chandel, Navdeep S. ROS Function in Redox Signaling and Oxidative Stress. Current Biology 24, R453-R462, doi:https://doi.org/10.1016/j.cub.2014.03.034 (2014).

58 Bjelakovic, G., Nikolova, D., Gluud, L., Simonetti, R. G. \& Gluud, C. Mortality in randomized trials of antioxidant supplements for primary and secondary prevention: Systematic review and meta-analysis. JAMA 297, 842-857, doi:10.1001/jama.297.8.842 (2007).

59 Raj, L. et al. Selective killing of cancer cells by a small molecule targeting the stress response to ROS. Nature 475, 231-234, doi:http://www.nature.com/nature/journal/v475/n7355/abs/nature10167-f1.2.html - supplementary-information (2011).

60 Zou, P. et al. Selective killing of gastric cancer cells by a small molecule targeting ROS-mediated ER stress activation. Molecular Carcinogenesis 55, 1073-1086, doi:10.1002/mc.22351 (2016).

61 Glasauer, A. Targeting SOD1 reduces experimental non-small-cell lung cancer. 124, 117-128, doi:10.1172/jci71714 (2014).

62 Wang, D., Malo, D. \& Hekimi, S. Elevated Mitochondrial Reactive Oxygen Species Generation Affects the Immune Response via Hypoxia-Inducible Factor- $1 \alpha$ in Long-Lived $<$ em $>$ Mclk1</em $><$ sup $>+/-</$ sup $>$ Mouse Mutants. The Journal of Immunology 184, 582-590, doi:10.4049/jimmunol.0902352 (2010). 
63 Kong, X. et al. Enhancing Nrf2 Pathway by Disruption of Keap1 in Myeloid Leukocytes Protects against Sepsis. American Journal of Respiratory and Critical Care Medicine 184, 928-938, doi:10.1164/rccm.201102-02710C (2011).

64 Thimmulappa, R. K. et al. Nrf2 is a critical regulator of the innate immune response and survival during experimental sepsis. The Journal of Clinical Investigation 116, 984-995, doi:10.1172/JCI25790 (2016).

65 Harman, D. Aging: A Theory Based on Free Radical and Radiation Chemistry. Science of Aging Knowledge Environment 2002, cp14- (2002).

66 Harman, D. Aging: a theory based on free radical and radiation chemistry. Journal of Gerontology 11, 298-300 (1956).

67 Balaban, R. S., Nemoto, S. \& Finkel, T. Mitochondria, Oxidants, and Aging. Cell 120, 483-495, doi:https://doi.org/10.1016/j.cell.2005.02.001 (2005).

68 Van Raamsdonk, J. M. \& Hekimi, S. Reactive oxygen species and aging in Caenorhabditis elegans: causal or casual relationship? Antioxidants \& Redox Signaling 13, 1911+ (2010).

69 Ristow, M. \& Schmeisser, S. Extending life span by increasing oxidative stress. Free Radical Biology and Medicine 51, 327-336, doi:https://doi.org/10.1016/j.freeradbiomed.2011.05.010 (2011).

70 Ryan, G. B. \& Majno, G. Acute inflammation. A review. The American Journal of Pathology 86, 183 (1977).

71 Zweifach, B. W., Grant, L. \& McCluskey, R. T. The Inflammatory Process. (Academic Press, 2014).

72 Coussens, L. M. \& Werb, Z. Inflammation and cancer. Nature 420, 860-867 (2002).

73 Pedersen, J., LaCasse, E. C., Seidelin, J. B., Coskun, M. \& Nielsen, O. H. Inhibitors of apoptosis (IAPs) regulate intestinal immunity and inflammatory bowel disease (IBD) inflammation. Trends in Molecular Medicine 20, 652-665 (2014).

74 Asquith, M. \& Powrie, F. An innately dangerous balancing act: intestinal homeostasis, inflammation, and colitis-associated cancer. Journal of Experimental Medicine 207, 1573-1577 (2010).

75 Neurath, M. F., Finotto, S. \& Glimcher, L. H. The role of Th1/Th2 polarization in mucosal immunity. Nature Medicine 8, 567-573 (2002).

76 Atreya, R. et al. Antibodies against tumor necrosis factor (TNF) induce T-cell apoptosis in patients with inflammatory bowel diseases via TNF receptor 2 and intestinal CD14+ macrophages. Gastroenterology 141, 2026-2038 (2011).

77 Su, L. et al. TNFR2 activates MLCK-dependent tight junction dysregulation to cause apoptosis-mediated barrier loss and experimental colitis. Gastroenterology 145, 407-415 (2013).

78 Günther, C. et al. Caspase-8 regulates TNF-[agr]-induced epithelial necroptosis and terminal ileitis. Nature 477, 335-339 (2011). 
Meijer, M. J. et al. Effect of the anti-tumor necrosis factor- $\alpha$ antibody infliximab on the ex vivo mucosal matrix metalloproteinase-proteolytic phenotype in inflammatory bowel disease. Inflammatory Bowel Diseases 13, 200-210 (2007).

80 Haep, L. et al. Interferon gamma counteracts the angiogenic switch and induces vascular permeability in dextran sulfate sodium colitis in mice. Inflammatory Bowel Diseases 21, 2360-2371 (2015).

81 Baumgart, D. C. \& Sandborn, W. J. Crohn's disease. The Lancet 380, 15901605 (2012).

82 Neurath, M. F. \& Travis, S. P. Mucosal healing in inflammatory bowel diseases: a systematic review. Gut 61, 1619-1635 (2012).

83 Taverniti, V. et al. Immunomodulatory effect of a wild blueberry anthocyanin-rich extract in human Caco-2 intestinal cells. Journal of Agricultural and Food Chemistry 62, 8346-8351 (2014).

84 Lau, F. C., Joseph, J. A., McDonald, J. E. \& Kalt, W. Attenuation of iNOS and COX2 by blueberry polyphenols is mediated through the suppression of NF$\kappa \mathrm{B}$ activation. Journal of Functional Foods 1, 274-283 (2009).

85 Kader, F., Rovel, B., Girardin, M. \& Metche, M. Fractionation and identification of the phenolic compounds of highbush blueberries (Vaccinium corymbosum, L.). Food Chemistry 55, 35-40 (1996).

86 Szajdek, A. \& Borowska, E. Bioactive compounds and health-promoting properties of berry fruits: a review. Plant Foods for Human Nutrition 63, 147 156 (2008).

87 Connor, A. M., Luby, J. J. \& Tong, C. B. Variability in antioxidant activity in blueberry and correlations among different antioxidant activity assays. Journal of the American Society for Horticultural Science 127, 238-244 (2002).

88 Schwabe, R. F. \& Brenner, D. A. Mechanisms of liver injury. I. TNF- $\alpha$-induced liver injury: role of IKK, JNK, and ROS pathways. American Journal of Physiology-Gastrointestinal and Liver Physiology 290, G583-G589 (2006).

89 McCarthy, E. T. et al. TNF-alpha increases albumin permeability of isolated rat glomeruli through the generation of superoxide. Journal of the American Society of Nephrology 9, 433-438 (1998).

90 Kim, J., Lee, S., Park, J. \& Yoo, Y. TNF- $\alpha$-induced ROS production triggering apoptosis is directly linked to Romo1 and Bcl-XL. Cell Death \& Differentiation 17, 1420-1434 (2010).

91 Matsuzawa, A. et al. ROS-dependent activation of the TRAF6-ASK1-p38 pathway is selectively required for TLR4-mediated innate immunity. Nature Immunology 6, 587-592 (2005).

92 Yamada, H. et al. LPS-induced ROS generation and changes in glutathione level and their relation to the maturation of human monocyte-derived dendritic cells. Life Sciences 78, 926-933 (2006).

93 de Souza, L. F. et al. Regulation of LPS stimulated ROS production in peritoneal macrophages from alloxan-induced diabetic rats: Involvement of high glucose and PPAR $\gamma$. Life Sciences 81, 153-159 (2007). 
94 Hwang, Y. S. et al. Interleukin-1 $\beta$ stimulates IL-8 expression through MAP kinase and ROS signaling in human gastric carcinoma cells. Oncogene 23, 6603-6611 (2004).

95 Lau, F. C., Bielinski, D. F. \& Joseph, J. A. Inhibitory effects of blueberry extract on the production of inflammatory mediators in lipopolysaccharide-activated BV2 microglia. Journal of Neuroscience Research 85, 1010-1017 (2007).

96 Faria, A. et al. Antioxidant properties of prepared blueberry (Vaccinium myrtillus) extracts. Journal of Agricultural and Food Chemistry 53, 6896-6902 (2005).

97 Haseeb, A., Chen, D. \& Haqqi, T. M. Delphinidin inhibits IL-1 $\beta$-induced activation of NF- $\kappa$ B by modulating the phosphorylation of IRAK-1Ser376 in human articular chondrocytes. Rheumatology 52, 998-1008 (2013).

98 Bae, C. H., Jeon, B. S., Choi, Y. S., Song, S.-Y. \& Kim, Y.-D. Delphinidin inhibits LPS-induced MUC8 and MUC5B expression through toll-like receptor 4mediated ERK1/2 and p38 MAPK in human airway epithelial cells. Clinical and Experimental Otorhinolaryngology 7, 198-204 (2014).

99 Garcia-Alonso, M., Minihane, A.-M., Rimbach, G., Rivas-Gonzalo, J. C. \& de Pascual-Teresa, S. Red wine anthocyanins are rapidly absorbed in humans and affect monocyte chemoattractant protein 1 levels and antioxidant capacity of plasma. The Journal of Nutritional Biochemistry 20, 521-529 (2009).

100 Kausar, H. et al. Berry anthocyanidins synergistically suppress growth and invasive potential of human non-small-cell lung cancer cells. Cancer Letters 325, 54-62 (2012).

101 Katsube, N., Iwashita, K., Tsushida, T., Yamaki, K. \& Kobori, M. Induction of apoptosis in cancer cells by bilberry (Vaccinium myrtillus) and the anthocyanins. Journal of Agricultural and Food Chemistry 51, 68-75 (2003).

102 Im, N.-K., Jang, W. J., Jeong, C.-H. \& Jeong, G.-S. Delphinidin suppresses PMAinduced MMP-9 expression by blocking the Nf- $\kappa \mathrm{B}$ activation through MAPK signaling pathways in MCF-7 human breast carcinoma cells. Journal of Medicinal Food 17, 855-861 (2014).

103 Hyun, J. W. \& Chung, H. S. Cyanidin and malvidin from Oryza sativa cv. Heugjinjubyeo mediate cytotoxicity against human monocytic leukemia cells by arrest of G2/M phase and induction of apoptosis. Journal of Agricultural and Food Chemistry 52, 2213-2217 (2004).

104 Decendit, A. et al. Malvidin-3-0- $\beta$ glucoside, major grape anthocyanin, inhibits human macrophage-derived inflammatory mediators and decreases clinical scores in arthritic rats. Biochemical Pharmacology 86, 1461-1467 (2013).

105 Bognar, E. et al. Antioxidant and anti-inflammatory effects in RAW264. 7 macrophages of malvidin, a major red wine polyphenol. PLoS One 8, e65355 (2013). 
106 Tsuda, T., Horio, F. \& Osawa, T. Cyanidin 3-O- $\beta$-D-glucoside suppresses nitric oxide production during a zymosan treatment in rats. Journal of Nutritional Science and Vitaminology 48, 305-310 (2002).

107 Zhang, Y. et al. Cyanidin-3-0- $\beta$-glucoside inhibits LPS-induced expression of inflammatory mediators through decreasing IкB $\alpha$ phosphorylation in THP-1 cells. Inflammation Research 59, 723-730 (2010).

108 Serra, D., Paixão, J., Nunes, C., Dinis, T. C. \& Almeida, L. M. Cyanidin-3glucoside suppresses cytokine-induced inflammatory response in human intestinal cells: comparison with 5-aminosalicylic acid. PloS One 8, e73001 (2013).

109 Pratheeshkumar, P. et al. Cyanidin-3-glucoside inhibits UVB-induced oxidative damage and inflammation by regulating MAP kinase and NF- $\kappa \mathrm{B}$ signaling pathways in SKH-1 hairless mice skin. Toxicology and Applied Pharmacology 280, 127-137 (2014). 


\section{Conclusion}

It has been previously reported that RCSC exhibits potent anticancer properties on multiple cell lines. For the first time, we have reported RCSC also functions as a potent anti-inflammatory and immune regulating agent on an in vitro inflammatory bowel disease model. RCSC was able to strongly regulate reactive oxygen species (ROS) signaling as well as prevent cytokine induced cell death. Additionally, several changes in gene expression were identified versus both the inflammatory and plant medium control. Specifically, NF-kB and SKI were up regulated, while CCL5, CD38, HLA1, and IKBKB were down regulated in NCM356. In CCD 841 CoN, CSF-2, GNLY, IL-1A, IL-1B, IL-6, IL-8, LIF, and PTGS2 were up regulated, and CSF-1, CXCL10, FAS, LY96, NFATC3, and SMAD3 were down regulated.

Extract of highbush blueberries were able to strongly regulate reactive oxygen species. In both NCM 356 and CCD $841 \mathrm{CoN}$, nuclear and cytoplasmic ROS were greatly reduced versus inflammatory control. Fluorescent microscopy analysis of nuclei in both cell lines showed less nuclear condensation versus inflammatory control as well. Flow cytometry analysis of CCD $841 \mathrm{CoN}$ revealed a significant reduction in cell death in blueberry treated groups following treatment with the pro-inflammatory cytokine cocktail.

RCSC was further analyzed for anticancer activity via fluorescent microscopy and flow cytometry using propidium iodide staining. RCSC acted as an efficient anticancer agent on SW620 in a dose dependent manner as previously reported. A dose response curve showed $\mathrm{EC}_{50}$ dilution factor to be 3.64 . Furthermore, toxicity was assessed in vitro 
on the normal colon cell line CCD $841 \mathrm{CoN}$. Roughly a 10-fold reduction, to 4.38 with the $\mathrm{EC}_{50}$ dilution factor, was observed.

A total of 25 fractions were collected via column chromatography in order to identify the bioactive constituents in RCSC. Fractions 10-18 had similar HPLC peaks, and eight individual peaks were identified in the pooled fractions. Peak number 6, was UV excited, which indicates possible conjugation with active functional groups, was poorly soluble in water, further indicating possible hydrophobic functional groups. RCSC fractions 10-18, when tested for anticancer activity in vitro on the colon adenocarcinoma cell line SW620, resulted in a similar response on cell death compared to whole RCSC. Toxicity was again assessed on the normal colon cell line CCD $841 \mathrm{CoN}$ by flow cytometry with propidium iodide staining and resulted in a similar, but slightly mitigated response compared to whole RCSC. Regulation of ROS by fractions 10-18 was similar in response to whole RCSC as well. In conclusion, RCSC acts as a potent anti-inflammatory and immune regulating agent. Moreover, highbush blueberry extract exhibited potent free radical scavenging capabilities. Lastly, RCSC fractions 10-18 are likely responsible for the anticancer, anti-inflammatory, and immune regulating functions of whole RCSC. 


\section{Future Directions}

Isolation and characterization of the compounds responsible for the effects of RCSC observed in the current study need to be identified. While recent published and unpublished work has indicated potent anti-cancer, anti-inflammatory, and immune regulating activity in RCSC, more research is needed to fully understand the biochemical and molecular mechanisms, specifically, in vivo testing in an animal model. Metabolomic, transcriptomic and proteomic analysis of inflamed cells treated with RCSC will lead to the identification of gene networks, protein-protein interactions and biological pathways involved in the process. Furthermore, docking studies involving differentially regulated metabolites and proteins will identify possible drug candidates, which can be used for further testing. This work and work of others has set the foundation for a novel method of in vitro generation of bioactive compounds with potent anticancer, anti-inflammatory, and immune regulating properties. While much work is still needed, this dissertation further outlines the beneficial role of secondary metabolites in rice callus suspension culture for human health. 